\title{
A Starting Mark of Health Promotion in Jakarta
}

\author{
Citation for published version (APA):
}

Sokang, Y. A. (2020). A Starting Mark of Health Promotion in Jakarta: Voicing the Community's Perspective. [Doctoral Thesis, Maastricht University]. Maastricht University. https://doi.org/10.26481/dis.20200528as

Document status and date:

Published: 01/01/2020

DOI:

10.26481/dis.20200528as

Document Version:

Publisher's PDF, also known as Version of record

\section{Please check the document version of this publication:}

- A submitted manuscript is the version of the article upon submission and before peer-review. There can be important differences between the submitted version and the official published version of record.

People interested in the research are advised to contact the author for the final version of the publication, or visit the DOI to the publisher's website.

- The final author version and the galley proof are versions of the publication after peer review.

- The final published version features the final layout of the paper including the volume, issue and page numbers.

Link to publication

\footnotetext{
General rights rights.

- You may freely distribute the URL identifying the publication in the public portal. please follow below link for the End User Agreement:

www.umlib.nl/taverne-license

Take down policy

If you believe that this document breaches copyright please contact us at:

repository@maastrichtuniversity.nl

providing details and we will investigate your claim.
}

Copyright and moral rights for the publications made accessible in the public portal are retained by the authors and/or other copyright owners and it is a condition of accessing publications that users recognise and abide by the legal requirements associated with these

- Users may download and print one copy of any publication from the public portal for the purpose of private study or research.

- You may not further distribute the material or use it for any profit-making activity or commercial gain

If the publication is distributed under the terms of Article $25 \mathrm{fa}$ of the Dutch Copyright Act, indicated by the "Taverne" license above, 


\section{A Starting Mark of Health Promotion in Jakarta: \\ Voicing the Community's \\ Perspective}

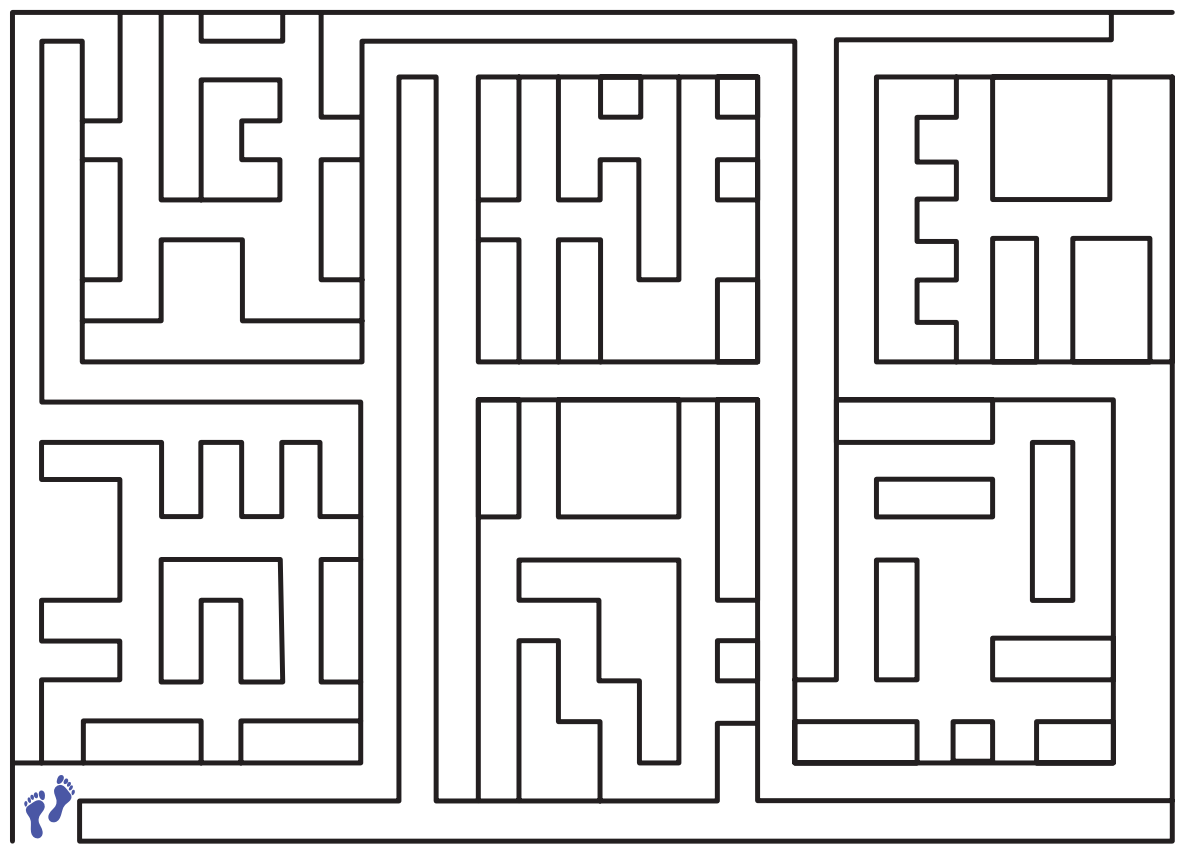

Yasinta Astin Sokang 


\title{
A Starting Mark of Health Promotion in Jakarta:
}

\author{
Voicing the Community's Perspective
}

Yasinta Astin Sokang 
(C) Yasinta Astin Sokang, Maastricht 2020

Cover design by: Yasinta Astin Sokang

Printed by: Proefschriftenprinten.nl

ISBN 978-90-830552-3-7

NUR 775

The research presented in this book has been financially supported by the Indonesia Endowment Fund for Education (Lembaga Pengelola Dana Pendidikan, LPDP), Ministry of Finance, the Republic of Indonesia. The views expressed in this book are the sole responsibility of the author and do not reflect the views of LPDP.

All rights are reserved. No part of this book may be reproduced, distributed, or transmitted in any form or by any means, without prior written permission of the author. 


\section{A Starting Mark of Health Promotion in Jakarta:}

\section{Voicing the Community's Perspective}

\section{DISSERTATION}

to obtain the degree of Doctor at the Maastricht University, on the authority of the Rector Magnificus, Prof.dr. Rianne M. Letschert, in accordance with the decision of the Board of Deans,

to be defended in public

on Thursday 28 May 2020, at 10:00 hours

by

Yasinta Astin Sokang 


\section{Supervisor}

Prof. dr. G. Kok

\section{Co-supervisor}

Dr. A. Westmaas, Hogeschool Leiden, the Netherlands

\section{Assessment committee}

Prof. dr. R.A.C. Ruiter (Chair)

Prof. dr. R.M.M. Crutzen

Prof. dr. J.E Prawitasari, Krida Wacana Christian University, Indonesia

Prof. dr. L. Schoonhoven, UMC Utrecht, the Netherlands

Dr. G.A. ten Hoor 


\section{Table of Contents}

Chapter 1. General Introduction

Chapter 2. From Physical to Spiritual: A Qualitative Study of Jakartans Health \& Sickness

Chapter 3. Jakartans' Perceptions of Health Care Services

Chapter 4. General Practitioners' Reflection on Health Promotion

Chapter 5. Considerations in the Community Coalition

Chapter 6. General Discussion

References

Summary/Samenvatting/Intisari

Summary

Samenvatting

Intisari

Valorization

Acknowledgements

Curriculum Vitae 


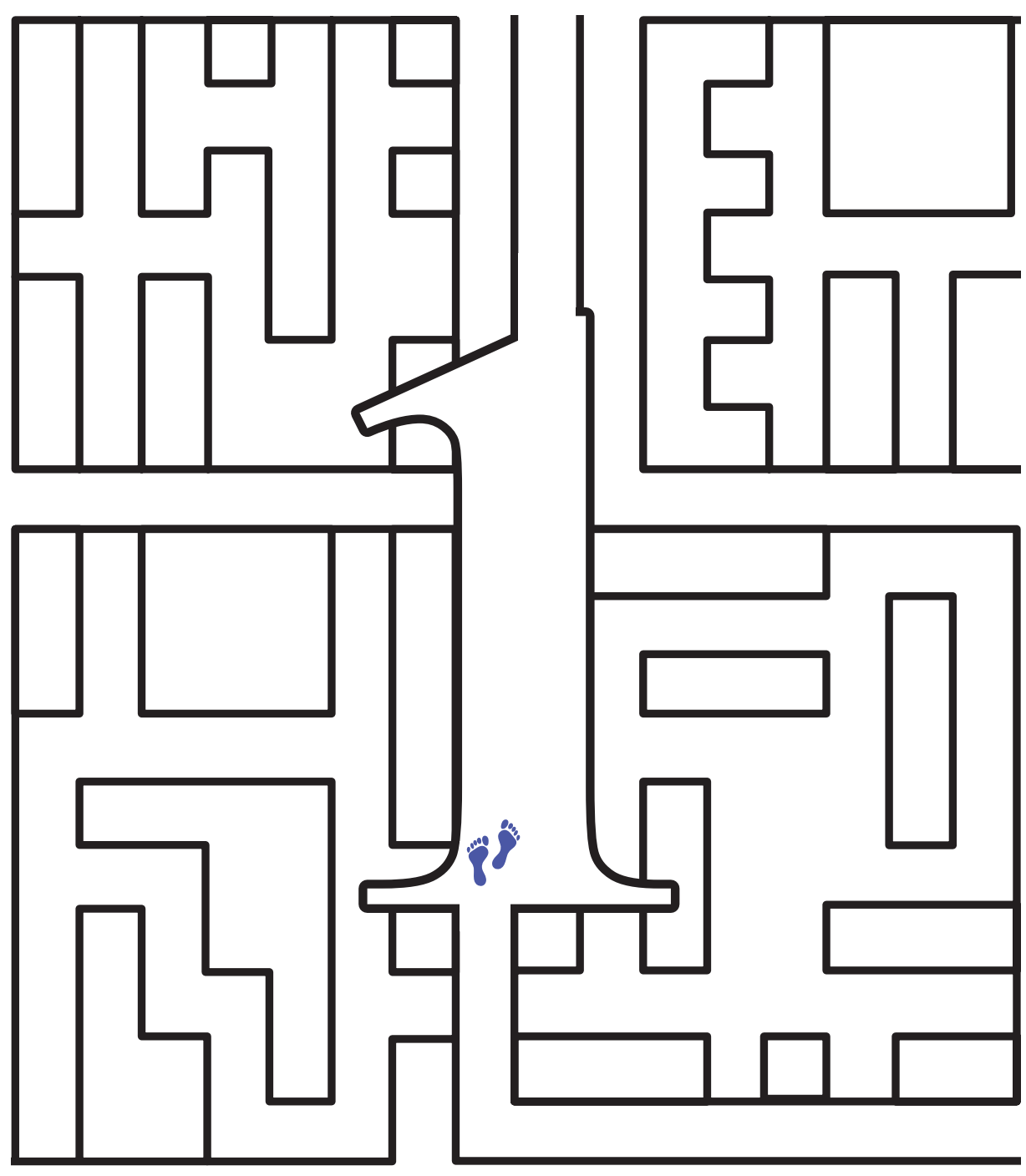




\section{Chapter 1.}

\section{General Introduction}




\section{Health promotion}

Health promotion efforts have been carried out in both developing and developed countries (Jack, Grim, \& Auld, 2012; Patel, Minas, Cohen, \& Prince, 2014; Watson \& Platt, 2000), with various approaches such as individual and group/community-based approaches (Cragg, Davies, \& Macdowall, 2013; Jackson \& Segal, 2014; K. L. Robinson, Driedger, Elliott, \& Eyles, 2006). According to the WHO, 'health promotion is the process of enabling people to increase control over, and to improve, their health' (WHO, 1998, p. 1). The purpose of health promotion is to positively influence the health status of individuals and communities by means of improving their health-related knowledge, attitudes, skills and behavior (Fertman, Allensworth, \& Auld, 2017; Hickson, 2015; Laverack, 2007). Additionally, health promotion is not only an action to improve skills and knowledge of the people, but also an action to change the social and environmental conditions (Cyril, Smith, \& Renzaho, 2016; Fertman et al., 2017; Gottwald \& GoodmanBrown, 2012). Together, health promotion refers to the movement to achieve an overall positive health, as opposed to a specific area of health care (MacDonald, 1998).

Although the term health promotion refers to any activities that improves health status (Fertman et al., 2017; Green, Cross, Woodall, \& Tones, 2019), the root of contemporary health promotion is health education (Green et al., 2019). This often results in people generalizing health promotion merely as health education. However, health promotion is more than health education, especially because illness and diseases are not preventable with health campaigns only (Lucas \& Lloyd, 2005; Snyder \& Hamilton, 2002). Studies have shown that increasing awareness on (un)healthy behavior was not necessarily followed by improvements in individual or community's healthrelated behavior (Bartholomew Eldredge et al., 2016). Consequently, it is crucial to conscientiously plan, implement, and evaluate a health promotion program in order to preserve the fundamental aspects of health promotion (Lucas \& Lloyd, 2005; Poland, Krupa, \& Mccall, 2009).

There are two key elements of health promotion in community settings, namely empowerment and community participation (Green et al., 2019). Empowerment refers to the feeling of the community members that they have the power to change or to make a difference in their health-related situation (Fertman et al., 2017; Green et al., 2019). The community needs to set a goal and make their own health-related decisions to increase their control over their health determinants (Cyril et al., 2016; Gottwald \& GoodmanBrown, 2012; MacDonald, 1998). Community participation is the involvement of the community members in health promotion programs, which includes defining the health needs, identifying the health problems, creating and evaluating the programs (Fertman et al., 2017; Motley, Holmes, Hill, Plumb, \& Zoellner, 2013; Reid et al., 2018). In line with this spirit of participation, health promotion practitioners/health promoters need to work in a partnership with health care professionals, community members, and policy makers (Fertman et al., 2017; Gottwald \& Goodman-Brown, 2012), because health promotion is a multisectoral task. It requires a holistic view on health, health promotion, 
and health care, both by the users and providers (Cyril et al., 2016; MacDonald, 1998; Poland et al., 2009).

To be able to implement the above-mentioned two key elements, it is essential for health promoters to adapt to the local values and needs, as well as to consider the social and cultural context of the community (Jongen, McCalman, \& Bainbridge, 2017). Recognizing the needs of the community accommodates health promoters to understand the relevance of their roles in the community (Bartholomew Eldredge et al., 2016; Gottwald \& Goodman-Brown, 2012). Thus, it is critical for the health care providers to develop health-services that cover the aspects considered important by the community (Fertman et al., 2017; Gottwald \& Goodman-Brown, 2012), in addition to the biomedical perspective that has been widely applied in health promotion programs and health services (Gottwald \& Goodman-Brown, 2012). Together, delivering a comprehensive health care system is more complex than simply delivering a health care system that -for example- focused only on one aspect, such as biomedical perspective, that tends to disregard the influence of social and cultural aspects of health-related issues (Gottwald \& Goodman-Brown, 2012; MacDonald, 1998).

\section{Cultural sensitivity in health promotion}

Research into complex health issues has shown that health-related behaviors are not independent but interrelated (Noar, Chabot, \& Zimmerman, 2008; Remenapp, Broome, Maetozo, \& Hausenblas, 2017). The interconnections between health-related behaviors make it difficult for health promoters and providers to target the determinants of behavior for people to live a healthy lifestyle. Furthermore, studies have found that health promotion interventions would be more effective if they matched the needs of the target group, because the intervention is tailored to the needs, as well as culturallyappropriate for the community (Kreuter \& McClure, 2004; Simmons, Bennett, Schwartz, Sharify, \& Short, 2002). Thus far, these studies suggested that one of the main obstacles to understand the determinants of health behavior is to understand the cultural context of determinants to develop an appropriate health promotion intervention.

Raising the issue of culturally-sensitive health promotion interventions is vital to provide a proper intervention. However, prior to that, it just as important to discuss what 'culture' is, especially if we consider the wide variation of terms associated with culture that are used both in practice and research. For example, culture is commonly defined as race and ethnicity, or used to describe particular socio-economic characteristics (e.g., the culture of poverty), to describe certain behaviors (i.e., drug culture, culture of violence, culture of fear) (Kreuter \& McClure, 2004; Voestermans \& Verheggen, 2013). In this dissertation, we define culture as the characteristics and knowledge of a specific group of people which is learned, reinforced, shared, transmitted intergenerationally, and reflected in the community's values, norms, beliefs, practices, patterns of communication, roles, and other social patterns (Betsch et al., 2016; Kreuter \& McClure, 2004; Pemunta \& Obara, 2012; Voestermans \& Verheggen, 2013). 
In relation to health promotion, developing a culturally-sensitive health promotion intervention is crucial as this type of promotion is considered to be respectful and sensitive to different values, needs and experiences of the community (Rowling \& Weist, 2004), because 'in practice, however, culture is more often assumed than assessed' (Kreuter, Lukwago, Bucholtz, Clark, \& Sanders-Thompson, 2003, p. 134). Successful health promotion interventions require the practitioners to be aware of, and sensitive to, the cultural values and beliefs of the target community, while paying attention to a culturally-appropriate approach or intervention (Fertman et al., 2017).

There are two main reasons why a culturally-sensitive approach is essential. First, health is multidimensional and is influenced by many factors such as culture, religious beliefs, and life experience (Gottwald \& Goodman-Brown, 2012). Every community has their own unique and culturally-based health-related attitudes, values, and beliefs that affect their health-related behaviors, both in positive and negative ways (Fertman et al., 2017; Simmons et al., 2002). In terms of health, people's values and beliefs include their concept of being healthy or sick, their beliefs about treatment -both traditional and modern health treatment- as well as health promotion, and their health care (Raza, Van De Poel, Panda, Dror, \& Bedi, 2016; Vaughn, Jacquez, \& Baker, 2009). A thorough understanding that people's health-related behaviors reflect their cultural and social values (Lucas \& Lloyd, 2005), and understanding that 'what is defined as unhealthy in one culture may be celebrated in another' (Cooper \& Thorogood, 2013, p. 23) will provide insights on how the community's cultural expectations and differences can affect their perception and experiences towards health, health promotion, and health care (Laverack, 2014).

Second, health promoters must acknowledge that communities have their own autonomy, including their right to preserve the cultural values in relation to their health. It is crucial for health promoters to understand that 'each and every person deserves dignity and has value' (Fertman et al., 2017, p.42). It also means that communities have specific needs and interests, as well as strength and benefit of their health-related culture (Simmons et al., 2002). The acknowledge toward community's autonomy will prevent health promoters to become judgmental and to blame community members for their unhealthy lifestyle (Gottwald \& Goodman-Brown, 2012). This is due to the fact that even though it passed down through generations, culture is dynamic, fluid, and adaptive (Betsch et al., 2016; Kreuter \& McClure, 2004; Pemunta \& Obara, 2012). At the same time, culture is a window of opportunity for health promoters to empower the community to change their unhealthy behavior (Smith, Tang, \& Nutbeam, 2006).

Additionally, culturally-sensitive research helps health promoters to comprehensively understand the cause and risk of health-related issues in a particular community, as well as their impact on health and disease outcomes (Thompson \& Gifford, 2000). Study by Kahissay, Fenta, \& Boon showed that community perceptions about health influences their actions on maintaining health, and treating illness, as well as their behavior in seeking treatment (Kahissay, Fenta, \& Boon, 2017). The community's perceptions of their health determined whether (and how) they would use health care services (Jayanthi, 
Suresh, \& Padmanaban, 2015; John \& Miller, 2015; Raza et al., 2016). By respecting the existing cultural frameworks, the health promoters would understand what and how people's health-related behavior is formed (Rowling \& Weist, 2004; Thompson \& Gifford, 2000). This is a more holistic approach, acknowledging that every culture has their own belief systems that may be different from the Western biomedical perspective (Jayanthi et al., 2015; John \& Miller, 2015; Vaughn et al., 2009).

To develop culturally-appropriate health promotion programs, health promoters need to build connection and trust with the community (Keleher \& Armstrong, 2005). In addition, health promoters need to be aware that a culturally-sensitive approach is not simply done by translating the language of the programs' curriculum and material to the community's language (Gardois, Booth, Goyder, \& Ryan, 2014; Netto, Bhopal, Lederle, Khatoon, \& Jackson, 2010). Furthermore, for the programs to be effective, health practitioners need to deeply focus on cultural competence and sensitivity from the start (Rowling \& Weist, 2004), instead of linking the implementation of health promotion programs to specific theoretical frameworks (Gardois et al., 2014).

There are two important dimensions of cultural sensitivity: the surface and deep structures (Resnicow, Baranowski, Ahluwalia, \& Braithwaite, 1999; Resnicow, Soler, Braithwaite, Ahluwalia, \& Butler, 2000). The surface structure focuses on the compatibility of health promotion programs materials and messages to the observable characteristics of the target community, such as the people, language, food, places, clothing, food, etc. It prioritizes acceptance of the health promotion program's messages by the community (Resnicow et al., 1999, 2000; Singelis, Garcia, Barker, \& Davis, 2018). The deep structure requires health promoters to understand the culture, historical, social, environmental and psychological aspects that are considered important by the target community, as well as their influence on the health-related behavior of the community (Resnicow et al., 1999, 2000). This means that health promoters need to understand the target community's perspectives and experiences in order to properly address the core cultural values of that community. Because of its complexity, this dimension is more elusive and received less attention (Resnicow et al., 1999, 2000; Singelis et al., 2018).

Health promotion programs that use a culturally-sensitive approach are very rare, especially in Indonesia. Most of the available programs were based on Western perspectives and approaches (Setiyawati, Blashki, Wraith, Colucci, \& Minas, 2014), which generalized the process of health-related behavior over groups and cultures (Bartholomew Eldredge et al., 2016; Raingruber, 2014). These Western perspectives do not take into account the cultural beliefs, social characteristics and (historical and social) environmental of health behavior (Barrera Jr., Castro, Strycker, \& Toobert, 2013; Raingruber, 2014). As a result, they often do not cover the cultural sensitivity issue in health promotion (Westmaas, 2013).

Due to the limited insights on the Jakartans (i.e., the citizens of Jakarta city) perspective of health and health care, as well as the lack of understanding in the health promotions' challenges in Jakarta, the present project uses a deep structure to understand 
Jakartans' perceptions of health and sickness, Jakartans' perceptions of health care services provided, and the challenges in conducting health promotion in Jakarta by the health workers. This dissertation utilized the Intervention Mapping (IM) framework, which provides an opportunity to incorporate the deep structure dimensions of cultural sensitivity while highlighting the empowerment and community participation element of health promotion. This is possible due to the ecological approach of IM (Bartholomew Eldredge et al., 2016).

\section{Intervention Mapping}

As discussed above, it is important to provide a community-based intervention model that approaches the issues not only theoretically but also in a culturally-sensitive way. IM is a health program planning approach that is based on using theory and evidence as the foundations to form an ecological approach to assess and intervene health problems and to engender community participation (Bartholomew Eldredge et al., 2016). One of IM's key points is that it provides clear descriptions to guide the process of planning and designing, developing, implementing, and evaluating the program objectives, supported by theory and empirical-evidence (Dalum, Schaalma, \& Kok, 2012; van Empelen, Kok, Schaalma, \& Bartholomew, 2003). IM consists of six steps with several tasks at each step (see Figure 1). The accomplishment of each task in a step leads to another task in another step of IM (Bartholomew Eldredge et al., 2016). The results of each step are described below.

Step 1 - The products of this first step are a description of a health problem, its impact on quality of life, behavioral and environmental causes, and determinants of behavior and environmental causes. Step 2 - The product of step 2 is a set of matrices of selected ecological levels (i.e., individual through societal) that combines performance objectives for each level, with selected determinants to produce change objectives, the most immediate target of the intervention. Step 3 - The product of step 3 is an inventory of behavior change methods selected to match each proximal program objective. Step 4 - The products of step 4 are the program materials. Step 5 - The product of step 5 is a detailed plan for accomplishing program adoption and implementation by influencing behavior of individuals or groups who will make decisions about adopting and using the program. Step 6 - The product of step 6 is the evaluation plan (Bartholomew Eldredge et al., 2016).

Intervention Mapping has been used to conduct numerous studies in various settings and communities, such as a school-based intervention to prevent obesity in children (Lloyd, Logan, Greaves, \& Wyatt, 2011) and a school-based fruit and vegetable promotion program (Pérez-Rodrigo et al., 2005). In work settings, IM has been used to develop a return-to-work intervention for temporary agency workers and unemployed workers sick-listed due to musculoskeletal disorders (Vermeulen, Anema, Schellart, van Mechelen, \& van der Beek, 2009), to design a workplace return-to-work program for occupational low back pain (Ammendolia et al., 2009), a weight gain prevention at the work site (Kwak et al., 2007), and a worksite physical activity intervention (Redding 
et al., 2008). Other studies have also focused on specific interventions on physical and psychological issues, such as a workplace intervention for sick-listed employees with stress-related mental disorders (van Oostrom et al., 2007), a chronic disease management program for stroke survivors (Sakakibara et al., 2017), a healthy diet intervention among type 2 diabetic patients (Mirzaei-alavijeh, Karami-matin, Van Lieshout, Mahboubi, \& Zinat-Motlagh, 2016), a program for the prevention of childhood obesity in 3 to 10 years old children (Verbestel et al., 2011), an adolescent smoking-cessation intervention (Dalum et al., 2012), and an intervention to improve diet and increase physical activity (Lloyd et al., 2011).

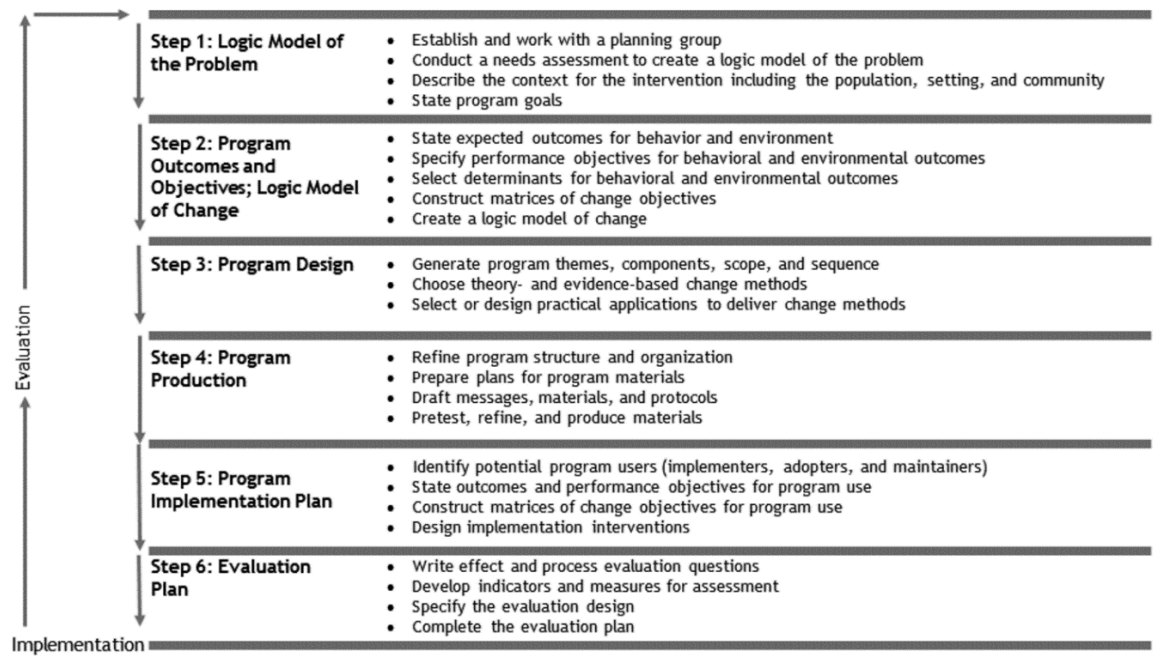

Figure 1: The Intervention Mapping protocol (Bartholomew Eldredge et al., 2016)

The above-mentioned studies showed different results, however, they all agreed that IM is a promising tool to develop a systematic theory-based intervention with a clear link between behavioral goals, theoretical methods, practical strategies and materials and with a strong focus on implementation and recruitment (Ammendolia et al., 2009; Dalum et al., 2012; Redding et al., 2008; van Oostrom et al., 2007; Vermeulen et al., 2009). IM also underlines collaborations with target groups as experts in describing the conditions and situations of the target group. One of the strengths of IM also lies in its flexibility so that users can incorporate different theories and approaches to reach the goal. These strengths make IM acceptable and feasible to apply in community programs (Ibrahim \& Sidani, 2014; Lloyd et al., 2011; Pérez-Rodrigo et al., 2005).

Existing studies have established that it is important for health care providers and promoters to conduct primary health prevention to improve public health status (Fertman et al., 2017; Suchert, Hanewinkel, \& Isensee, 2015; Treerutkuarkul \& Gruber, 2015). Furthermore, data from several studies suggested that healthy/unhealthy behaviors are related to each other (Jormfeldt, Svedberg, Fridlund, \& Arvidsson, 2007; Kahissay et 
al., 2017). At the same time, health care providers and promoters need to pay attention to the community's specific needs (Fertman et al., 2017; Jormfeldt et al., 2007; Suchert et al., 2015). IM meet these requirements for developing an ideal intervention because of its ability to guide theory-based health intervention planning while at the same time relating to the cultural uniqueness of the target community (Bartholomew Eldredge et al., 2016).

In this dissertation, we used IM as a framework during the project, especially the first step of the framework. IM forces the user to think of each step thoroughly, to focus on the open-minded approach when approaching different cultures, and to create the room to discuss and involve the community in every step of the protocol by its transparency (Bartholomew Eldredge et al., 2016; Dalum et al., 2012). This approach also consider both theory- and evidence-based insights (Bartholomew Eldredge et al., 2016).

\section{Health and health promotion in Jakarta}

\section{Health promotion in Indonesia}

Indonesia has two types of health providers, namely private and public health care providers. Private health providers are non- or for-profit providers, as well as individual doctors and midwives who have their own private clinics. The responsibility for financial support and the management of the private health providers lie with the providers themselves (Mahendradhata et al., 2017). In 2019, around 63.5\% of hospitals in Indonesia are managed by private providers (Dorimulu, 2019). Meanwhile, public health providers are managed and financially supported by the Indonesian government (Mahendradhata et al., 2017), with its primary health service centers known as Community Health Centers (CHCs; Indonesian: Pusat Kesehatan Masyarakat, Puskesmas). CHCs are considerably important because they are the frontline institution in providing health services to communities in every sub-district in Indonesia (Agustina et al., 2019; Mahendradhata et al., 2017). Each CHC has five priority programs, which includes: a) health promotion services; b) environmental health services; c) maternal, child and family planning services; d) nutrition services; and e) prevention and disease control services (Ministry of Health Republic of Indonesia, 2014a). The minimum number of the staff at a CHC is 31 people. This includes medical and non-medical staff. Medical staff are general practitioners or doctors, dentists, nurses, midwives, public health staff, environmental health workers, laboratory technician, nutritionist, and pharmacists. Non-medical staff are administrative staff, including financial administration staff, information systems staff, and other operational support staff (Health PPSDM Agency, 2015; Ministry of Health Republic of Indonesia, 2014a).

To improve the health status in Indonesia, the government provides public insurance and increases the number of health facilities such as CHCs (Ministry of Health of the Republic of Indonesia, 2015b; Statistics Indonesia, 2013). However, in performing its programs, CHCs encounter many problems, such as the lack of medical staff, the lack of 
skills and knowledge, the lack of technology, and the difficulty of reaching communities due to the large areas of coverage (WHO, 2014; WHO Country Office Indonesia, 2016). For example, in 2015, 16 general practitioners or doctors, 5 dentists, 88 nurses, and 44 midwives were responsible to treat 100,000 people (Agustina et al., 2019). It is less than the $50 \%$ ideal ratio of health workers to population (Agustina et al., 2019; Mahendradhata et al., 2017). As a result, patients cannot be treated properly or are not treated at all because they choose to go home due to the long waiting period (Putri, 2013). The lack of operational funds had been another problem. However, in 2015, all CHC in Indonesia got a budget of 4 trillion IDR to focus on promotive and preventive programs (Tarigan, 2015). The Ministry of Health evaluates the CHC performance by accrediting certification as a benchmark of success of $\mathrm{CHC}$ in performing health promotion program. However, the focus of the assessment of the accreditation is physical health, which is the fifth priority program. At the same time, the focus on physical health may foreshadow other health issues in the community which also include psychological problems (Abdullah, Hort, Abidin, \& Amin, 2012). The accreditation may influence CHC staff to be more focused on physical health prevention than other aspects of health while conducting their prevention programs. In addition, the health promotion approaches by the CHC often implement a typical biomedical approach which sees health/sickness as the cause and the effect of the physical world (Balog, 2005; Rheenen, 1998). The lack of understanding of how people perceive their health means that efforts to improve the health status might not match the public need (Jormfeldt et al., 2007; Mendelson, 2002).

Despite the presence of private and public health providers, Indonesians do not always use these health facilities. They tend to delay visiting health care providers up to ten days after the first onset of symptoms of their illnesses (Suswardany et al., 2017). In many cases, patients do self-treatment by consuming herbal medicines, using traditional treatment or buying medicine from private drug sellers (Karyana et al., 2016; Mahendradhata et al., 2017; Suswardany et al., 2017).

Like many other global south countries, Indonesia has a strong tradition of native medicine, and the people often use the traditional medicine rather than visiting modern health professionals (Mahendradhata et al., 2017; Marthoenis, Aichberger, \& Schouler-Ocak, 2016). Studies have shown that around $30.4 \%$ of Indonesian families used traditional treatment and medicine for primary health care purposes (Nurhayati $\&$ Widowati, 2017). The main reason was that the traditional care was closer to their homes, was accessible and more affordable (WHO, 2013). Furthermore, the practice is culturally-acceptable and trusted by the community, as it fits the community's everyday life and belief systems (Nurhayati \& Widowati, 2017; WHO, 2013).

\section{Health in Jakarta}

Jakarta is the most multicultural city in Indonesia whose inhabitants have different cultures, ethnicities, religions, and social and financial status (Cybriwsky \& Ford, 2001; Hadiwinoto \& Leitmann, 1994; McCarthy, 2003; Statistics Indonesia, 2010, 2015). Despite being the capital city of Indonesia, Jakarta's public health status is no better 
than that of other areas of Indonesia. Some diseases, such as tuberculosis, measles, cancer, diabetes, and abdominal obesity, have higher incidence rates in Jakarta than other provinces (Ministry of Health of the Republic of Indonesia, 2015c; Ministry of Health Republic of Indonesia, 2013, 2016).

A survey of the Ministry of Health of the Republic of Indonesia in 2015 may explain the causes of the health problems in Jakarta: the lack of sanitation, and poor diet. Proper sanitation in residential area has only been implemented in five of the city's 267 subdistricts (Ministry of Health Republic of Indonesia, 2016). The sanitary quality of public spaces in Jakarta is also low. Jakarta has 633 public places, yet only 185 of them met the national health and sanitary requirements (Ministry of Health Republic of Indonesia 2016). Other important factors include poor drainage systems and littering, which lead to the rivers being heavily polluted, which in turn cause frequent flooding (Conron, 2014; Ismail, 2015; Jong, 2015; Pasang, Moore, \& Sitorus, 2007; Steinberg, 2007). Poor air and water quality due to pollution also adds to the list of causes of health problems in Jakarta (J. Hays, 2008; Samosir, 2015).

Poor diet is the second major cause of health issues in Jakarta. A survey on basic health conducted by the Ministry of Health of the Republic of Indonesia (2013) have earlier suggested that Jakartans consumed energy $>130 \%$ of the Indonesian nutritional rate standard (Ministry of Health Republic of Indonesia, 2013). Indonesians prefer instant foods and beverages than vegetables, and they spend more money on tobacco and betel/ areca nuts than on fish/shrimp/shell/meat, and eggs and milk (Apsari et al., 2016).

In contrast to the survey about the lack of sanitation and poor diet that periodically reported, there are few reports of physical activities or exercises undertaken by Jakartans. Information from the Ministry of Health Republic of Indonesia (2016) does not provide complete data about physical activities. The available data cover the number of primary health service centers, which provide physical activities to communities in Jakarta. These physical activities were only provided by 16 of the 340 primary health service centers in Jakarta, despite the fact that studies have shown the importance of physical activities or exercises (Amato, Park, \& Nigg, 2016; Brug, Oenema, \& Ferreira, 2005; Mays et al., 2012; Sorensen, Emmons, Hunt, \& Johnston, 1998). The report from a survey conducted by PT Cerebos Indonesia among 1,000 professionals between 25 and 45 years of age showed that of those respondents, $96 \%$ were aware that good health could help their careers, but only half of them actually had a healthy lifestyle (Dewi, 2015). The data showed that more than a third of respondents spent more than 10 hours in the office per day and 6 in 10 respondents spent two to four hours per day on the road. Up to $23.8 \%$ of professionals, most of whom were women, admitted that they did not exercise at all (Dewi, 2015; Pradesha, 2015).

The studies that are featured in this dissertation were conducted at Grogol Petamburan sub-district located in West Jakarta Municipality. As for the governmental structures, under the municipal of government, there are four sub-levels, which are sub-district (Indonesian: kecamatan), village (Indonesian: kelurahan), community association (Indonesian: rukun warga, RW), and neighborhood association (Indonesian: rukun 
tetangga, RT). While the heads of sub-district and village are chosen by formal election, the heads of community association and neighborhood association are chosen by community's informal agreement based on the willingness of the chosen person (Pasang et al., 2007).

The Grogol Petamburan sub-district has 225,038 inhabitants in an area of $9,99 \mathrm{~km}^{2}$ (around 22,526 people/ $\mathrm{km}^{2}$ ). The inhabitants divided into 74,672 families, 7 administrative villages, $868 \mathrm{RT}$, and $73 \mathrm{RW}$ (Statistics coordinator of Grogol Petamburan sub-district, 2016). Grogol Petamburan has 8 CHC, 5 general hospitals, 3 maternity hospitals, 15 private polyclinics, and 32 pharmacies. A total of 17 nurses, 23 midwives, 13 pharmacists, and 9 other medical staff serve the Grogol Petamburan sub-district health needs. It means that one CHC serves around 28,130 people, and one medical staff serves around 3,630 people (Statistics coordinator of Grogol Petamburan sub-district, 2016).

The inhabitants of Grogol Petamburan come from different cultures, ethnicities, and religions with 2,906 people coming and 3,155 people moving only in 2015. More than three-quarters of populations $(76,06 \%)$ are in productive age (15-64 years old). Based on the job, 83,811 people are employees whose work in textile industries (convection, or garment), food and beverage industry, printing and publishing industry. As many as 24.279 people work as traders, and 15,148 people are entrepreneurs. A total of 12,067 people are civil servants and 572 are Indonesian army/police. Despite all the jobs, there are 5,086 poor families in this sub-district (Statistics coordinator of Grogol Petamburan sub-district, 2016).

Unfortunately, despite the complexity of the health-related issues in Jakarta, to our best knowledge, international peer reviewed publications on health and health promotion in Jakarta are limited. This results in the lack of knowledge and understanding of the concept of health and health promotion from the Jakartans' point of view. This point of view is of importance for the government, health care providers, and policy-makers to develop health promotion programs, policies and regulations. As a consequence of the lack of the community's perspectives, the health care services provided in Jakarta tend to use a biomedical perspective in treating their patients (Jayanthi et al., 2015), in spite of Jakarta's status as a multicultural city with inhabitants coming from various backgrounds and cultural values that merge into one (Simone, 2014). Hence, this dissertation was prepared as the first step to assess Jakartans' perception towards health and health promotion while highlighting the cultural context of the issue.

\section{Methodology}

\section{Needs assessments}

As described earlier, the project focused mainly on the first step of IM, which is the needs assessment phase. A needs assessment should be the point of departure of every health promotion initiative (Cragg, Nutland, \& Hickson, 2015; Laverack, 2014; Smith 
et al., 2006). It is a systematic process to identify the gap between the current and the ideal situation of an individual or a group (Gilmore, 2012). However, the most important point of the need assessment process is to understand the perspectives of the community. In terms of healthy behavior, the need assessment is crucial for the researchers to understand the character of the community, such as the quality of life of its members, the health needs, concerns, and priorities, and the causing of the behavioral and environmental conditions (Bartholomew Eldredge et al., 2016; Gilmore, 2012; Stephens, 2008). Moreover, during the process of needs assessment, health promoters can identify the strength and resources of the community, such as human, social, physical environment, and cultural assets (Laverack, 2014; Stephens, 2008). This is the health promoter's opportunity to empower the community (Smith et al., 2006). The inventory of the strengths and resources can guide us to build an intervention by using these resources to improve the community's health (Bartholomew Eldredge et al., 2016). Therefore, the needs assessment process do not only involve the identification of needs, but also the identification of solutions and actions to resolve the needs in the form of appropriate interventions (Laverack, 2007). To build a culturally-appropriate and relevant intervention, it is critical for health promoters to understand the community's sociocultural background (Fertman et al., 2017; Simmons et al., 2002) by integrating all the community stakeholders' perspectives during the need assessment processes (Gregg \& O’Hara, 2007; Kruger, Lewis, \& Schlemmer, 2010; Stephens, 2008).

As indicated earlier, understanding all the community stakeholders' perspectives also means understanding the causes of and the possible solutions for the ongoing health issues. When health promoters are aware that the community is empowered, they shall see that the community has its own strengths and positive values. Thus, in conducting the need assessment process, the health promoters are expected to not focus on the negative cause of the issue but to prioritize the distinctive strength characteristics of the community in order to build an effective (blueprint for a) health promotion intervention. On the other hand, health promoters also need to carefully consider who are the stakeholders in the community, who are the key persons, and invite group representatives to avoid over-power or domination of certain groups during data collection (Laverack, 2007).

Information may come from the community members and representatives in many ways (Stephens, 2008). Consequently, the scope of needs assessment in health promotion must be broad, and reflect an open mind that the community's health issues are related to many other factors, such as physical, social, and cultural context in which people live (Smith et al., 2006). An open mind is needed to avoid existing perspectives that might influence the questions raised and the desired information from the process of needs assessment (Fertman et al., 2017; Kreuter \& McClure, 2004). 


\section{Methods}

To understand the stakeholders' perspective on the health-related issue, qualitative methods are the most revealing approach to help health promoters (Bartholomew Eldredge et al., 2016; Laverack, 2014; Nutlands \& Cragg, 2015). Methods such as surveys, interviews, or focus groups can be used to interrogate the community's needs and views on health-related issues (Laverack, 2014; Nutlands \& Cragg, 2015; Stephens, 2008). In the first study, we used an open-ended survey. The second and the third study used FGD (Focus Group Discussion) approach, while the fourth study was a literature review study. Qualitative approach was used as it is a well-established approach to map ideas and feelings, as well as to explore the problems and the solutions.

Even though there are many publications about the determinants of healthy lifestyle, our project aims to accept the participants' perspective as open-mind as possible without interference of our perspective (C. Anderson, 2010; Harwell, 2011). The method used was focused on the discovery and understanding of an issue from the perspectives of the community it involves. The FGD provided an opportunity to explore culturally-specific information, such as the beliefs, points of view, behaviors, and social norms of the community member (Finch \& Lewis, 2003). Additionally, an FGD is an effective way to observe the group dynamics and to understand the interaction among members in the community without having to interfere with the researcher's perspective (C. Anderson, 2010; Harwell, 2011). The questions in the FGD's were open-questions. Using open questions, we respected the cultural value in the community, which includes the religious value, so that we discovered and understood the community's experiences, perspectives, and thoughts as close as the community see it.

\section{Objectives and research questions}

The overall aim of this dissertation is to understand the values that are considered important for health and health promotion by the communities, which include the health workers, while paying attention to the cultural context of the issue.

The research questions of this dissertation are:

1. What do health and sickness actually mean to Jakartans?

2. How do the health care users in Jakarta perceive the quality of health services provided?

3. What are the challenges and obstacles on conducting health promotion programs in Jakarta from the perspective of the health workers?

4. What are the important aspects in applying the Community Coalition (CC) approach for health promotion intervention in urban areas? 


\section{Overview of the dissertation}

The chapters of this dissertation represent the order of the four studies which were conducted to achieve these projects' goal. In Chapter 2, we conducted a study to understand the meaning of health and sickness among Jakartans. The result of the study confirmed that health providers in Jakarta focused more on the physical approach, while the community considered other aspects of health. For example, the spiritual aspect, the ability to do daily activities aspect, and even the combinations of several aspects, such as a combination of the physical, psychological, and spiritual aspects, or a combination of the physical, psychological, and ability to do daily activities aspects. In Chapter $\mathbf{3}$, we conducted an assessment to evaluate how health care users perceived the quality of health care providers in Jakarta. In this study, we found that the community has a rather negative perspective toward the CHC's, which are government-mandated health centers. This negative perspective leads to a tendency of the community to do selftreatment before visiting the health center. To understand both sides of the issue, we studied the perspectives of health workers on how they performed their services, in Chapter 4. We found that health care workers were dissatisfied with their own health programs. They were understaffed, because they needed to provide services inside and outside of the $\mathrm{CHC}$ with a high number of patients. Another challenge for health workers was that the community members preferred to apply self- and traditional treatments for health-related issues. This challenge was in line with our previous finding. The health workers suggested that the $\mathrm{CHC}$ needs to enlist the help of community leaders, clinical psychologists, and religious leaders to assist the health promotion programs at the health centers. With this suggestion in mind, we found from the literatures that a Community Coalition might be an ideal approach that fits the suggestion to involve multiple parties in health promotion programs. Thus, in Chapter 5, we conducted a literature review to synthesize aspects that are considered important in applying $\mathrm{CC}$ approaches in health promotion intervention in urban areas. The review suggested that there are seven aspects that are considered important, namely the members' diversity, the goal clarity, the member's interest and motivation, the leadership, the trust and cohesion, the role clarity, and understanding the norm and the culture of the community. Finally, Chapter 6 contains a general discussion of the projects. 


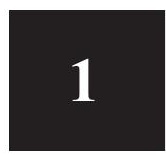




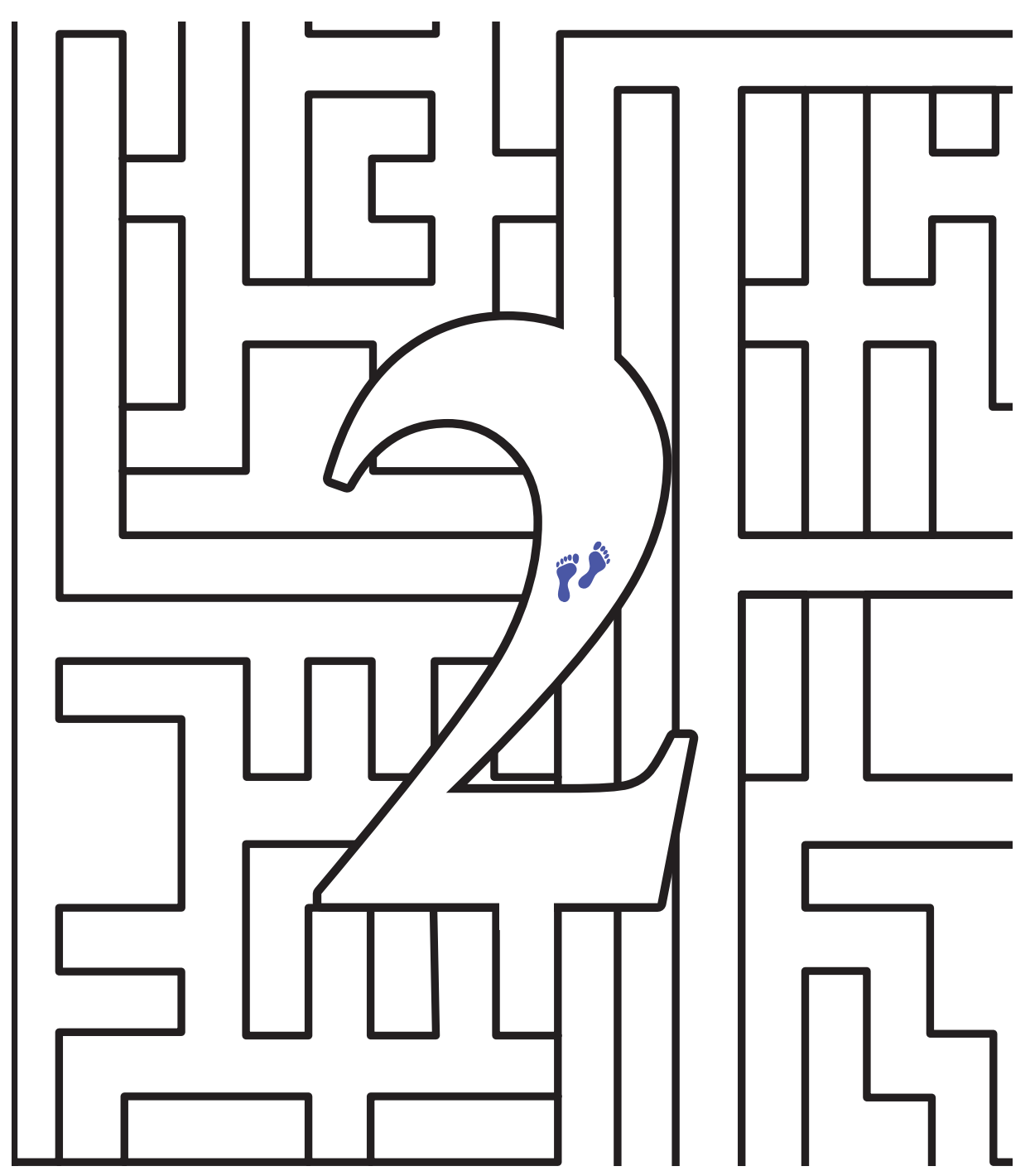




\section{Chapter 2.}

\section{From Physical to Spiritual: A Qualitative Study of Jakartans Health \& Sickness}

Based on:

Sokang, Y. A., Westmaas, A. H., \& Kok, G. (2019). From Physical to Spiritual: A Qualitative Study of Jakartans Health \& Sickness. International Journal of Environmental Research and Public Health, 16(19), 3564. https://doi.org/10.3390/ijerph16193564 


\begin{abstract}
Understanding the perceptions of health and sickness can help the government, health providers and health promoters encourage individuals to participate in healthy behaviors and to follow a healthy lifestyle. Jakarta, the capital city of Indonesia, is a culturally, socially and financially diverse city, with complex health care needs. As yet, there is no published data available about Jakartans' (i.e., the citizens of Jakarta city) perceptions on health and sickness. This study aimed to describe what health and sickness mean to Jakartans. To this means, we collected data using an open-ended survey about the meanings of health and sickness from 640 Jakartans. Five main themes of health and sickness emerged. The five themes of health were health as a physical condition, a psychological condition, a spiritual condition, a capability to carry out daily activities, and a healthy lifestyle. The themes regarding sickness were sickness as a physical condition, a psychological condition, an abnormal circumstance or bad situations, a spiritual condition, and an inability to carry out daily activities. We discussed how the above-mentioned perceptions might influence the daily health-related behaviors of Jakartans. In contrast to the typical biomedical approach, we found that, in Jakarta, health was not merely seen as a causal effect of the physical world. Further details are discussed.
\end{abstract}




\section{Introduction}

In Indonesia, improving the health condition has been a continuous effort (Ministry of Health of the Republic of Indonesia, 2015a). For example, the number of health facilities has increased each year from 9.665 units in 2013 to 9.731 units in 2014 (Ministry of Health of the Republic of Indonesia, 2015b). However, these health facilities are often unable to provide optimal health supports due to the limitation of human resources (Ministry of Health of the Republic of Indonesia, 2015a). Moreover, providing health services by increasing the number of health facilities requires a significant investment in terms of finances and time (McPake et al., 2015). Therefore, it has been argued that in a highly-populated country such as Indonesia, preventive health care-for example, health promotion programs - is a better way to improve health quality as compared to curative approaches such as increasing the number of health facilities (Treerutkuarkul \& Gruber, 2015).

In order to implement an effective health promotion, it is important to understand health in its cultural context. People's perceptions about health influence their actionsfor example, the behaviors they participate in and whether they have a healthy lifestyle (Kahissay et al., 2017). It has been shown in studies that patient perceptions of their current health problems in part determine whether (and how) they would use health care services (Jayanthi et al., 2015; John \& Miller, 2015; Raza et al., 2016). A better understanding of the cultural context of health perceptions can help health care providers to provide the type of health care that meets the needs of the citizens, to improve the communication between health care providers and patients, and to improve patient satisfaction (Jormfeldt et al., 2007; Kahissay et al., 2017).

Indonesians tend to interpret health not only in term of the physical condition but also in terms of other aspects. These aspects include art, which is defined by the chance to enrich and express one's self (Hidajat, 2005); religiousness, which defines health as a gift from God, a blessing (Liem \& Yuniarti, 2012); an uncomfortable feeling, which is defined by being unable to work, losing appetite, and not having any money ('kantong kering') (Soejoeti, 2008).

Although many studies on the perception of health have been conducted in various geographic areas of Indonesia, there has been (to our knowledge) no international, peer reviewed publication on the perceptions of health and sickness of Indonesian; let alone a publication on the perceptions of health and sickness of the citizens of Jakarta (henceforth will be called "Jakartans"), despite of the fact that Jakarta is the most multicultural city in Indonesia. As the capital, Jakarta is the most populated city in Indonesia (Statistics Indonesia, 2015). This dense population brings out multiple problems such as poor drainage systems and waste problems, which cause frequent flooding (Jong, 2015). Such problems are detrimental both financial- and health-wise. As a consequence, health care needs have been increasing enormously (Ministry of Health of the Republic of Indonesia, 2015a). Given the aforementioned benefits of preventive health care, an ideal solution would be to provide health care by means of public health promotion. 
As discussed above, it is important to provide an effective health promotion that approaches the issues not only theoretically but also in a culturally sensitive way. Intervention Mapping (IM) is a health program planning approach that is based on using theory and evidence as the foundations for an ecological approach to assess and intervene health problems and to engender community participation (Bartholomew Eldredge et al., 2016). One of IM's key points is that it provides clear descriptions to guide the process of planning and designing, developing, implementing, and evaluating the program objectives, supported by theory and empirical-evidence (Dalum et al., 2012; van Empelen et al., 2003). IM consists of six steps with several tasks at each step.

In order to provide an effective health promotion, as an initial step, this study focused on the Step 1 of IM. Step 1 of IM highlights the importance of assessment to understand the behavior and environmental determinant, as well as to understand the characteristics of the community, including the social perspective of a community at risk for healthrelated issues. Therefore, the purpose of this study is to describe what health and sickness actually mean to Jakartans. This information can be used by the government, health care providers, health promoters, and nurses to ensure that health care services not only provide good care, but also to meet the specific needs of the inhabitants of Jakarta.

\section{Materials and Methods}

The qualitative descriptive approach was used to capture the participants' perception of health and sickness. The approach was used as it provides direct descriptions of the target phenomenon. The participants explain their experiences in their own words and presented the facts of the perception in everyday language (Bradshaw, Atkinson, \& Doody, 2017; Sandelowski, 2010; Willis, Sullivan-Bolyai, Knafl, \& Cohen, 2016). This approach enables us to address the cultural values of health and sickness perception.

\section{Settings and Respondents}

The data were collected by means of an open-ended survey which was adapted from Liem \& Yuniarti (Liem \& Yuniarti, 2012). This survey comprises two statements which designed to assess the respondents' perceptions of health and sickness. Specifically, respondents were asked to complete the sentences 'Health is...' and 'Sickness is...' based on their own perceptions. This sentence completion was chosen to provide the opportunities to respondents to use their own words to explain their perceptions (Clarke, Hayfield, Moller, \& Tischner, 2017). The flexibility of this approach also allowed respondents to make a wide variety of responses, which in turn provided us a rich dataset. The data were collected in multiple rounds. At the end of each round, an initial analysis was conducted to identify the emerging themes. Data collection was stopped when the saturation of the data was achieved; no new themes emerged from the data (Aldiabat \& Le Navenec, 2018; Saunders et al., 2018). The raw data supporting 
the conclusions of this manuscript will be made available by the corresponding author, without undue reservation, to any qualified researcher.

The study was conducted by the first author in Jakarta. With a total area of 1324.66 $\mathrm{km}^{2}$, and an estimated population of 10 million people in 2015 (Directorate General of Public Administration, 2015), it is the most highly populated city in Indonesia. The inhabitants come from different areas and ethnic backgrounds, and they bring their own culture and values to the city (Simone, 2014). Respondents in this study were recruited from September 2014 until November 2014 in all areas of Jakarta. Respondents were included in the study if they were born in or had residence in Jakarta and were of age 12 or older. The respondents were recruited using a convenience sampling.

Five research assistants were trained by the first author to collect the data and to answer any questions potential respondents might have. Upon finishing their training, the research assistants went to public areas such as shopping malls, markets, streets, hospitals, and schools in order to find potential respondents. Each potential respondent was briefly informed about the purpose, procedures, and confidentiality of the study. After respondents had agreed to participate by providing an oral consent, they received a survey package containing the questionnaire, a pen and a clipboard. The questionnaire included a written consent, information about the purpose and confidentiality of the study, and information about members of the research team (name, faculty, university, and email address). For respondents who are underage (under 16 years old (y/o)), the written consent was given by the parents, or the school principle and/or teacher. Together, written informed consent was obtained from all adult participants and the parents/ teachers of non-adult participants. The respondents were instructed that there were no wrong answers, and they should complete the sentences based on what first came into their mind. In addition to completing the two open-ended questions, respondents filled in four demographic questions about sex, age, education level, and income/allowance per month. After the surveys were completed, respondents were asked if they had any questions and thanked for their participation.

The study was approved by both the Ethical Review Committee of the School of Psychology and Neuroscience, Maastricht University (Reference number of research line: ECP-04-09-2012), and an internal peer reviewer of the Center for Research and Community Service of Krida Wacana Christian University, Jakarta (Contract number: 04 /UKKW/LPPM-FPSI/Lit/I/2014). The written informed consent was obtained from all adult participants and the parents/teachers of non-adult (under 16y/o) participants.

\section{Analysis}

All data were analyzed using content analysis with an inductive approach. This approach was used because there had been little to no existing information about the meaning of health and sickness from the perception of Jakartans. The analysis process included open coding, creation of categories, and abstraction (Mayring, 2014). In the open coding part, the researchers applied human coding for this study and used the Indonesian Dictionary to compare the meaning of the words. The open coding and categorization were carried 
out by two teams of three researchers. Each team conducted the open coding of all data by means of discussion within the team. The coding rules are described in more details in the Appendix A, B, and C.

In the creation of categories part, the coded words were categorized in groups of similar statements. Subcategories with a similar word content across the teams were grouped into categories (Charmaz, 2012). If a statement did not fit an existing group, a new group was formed. Lastly, in the abstraction part, the resulting groups were discussed between teams in order to create themes. The categories with a similar theme were grouped into main themes and named using words that respondents had generated (Hussein, Hirst, Salyers, \& Osuji, 2014).

\section{Results}

In total, 640 Jakartans ( $51 \%$ females) Jakartans completed the study. There were no data dropped out as the participants filled the sentences completely. Potential participants who refuse to participate mainly because they were in a rush or had no time to join the study. The sample has a mean age of $\mathrm{M}=23.22$ (range: 12-60 years). Although respondents from all educational levels participated, people with a senior high school education were over-represented (see Table 1). The demographic distributions correspond to that of the research assistants, which might explain the overrepresentation. Although the instruction was to look for respondents of various ages, the research assistants' age might influence the reactions given by the prospective respondents.

We categorized the meanings of both health and sickness in five main themes (see Tables 2 and 3). Each main theme will be described with regard to the 'single term' and the 'in combination' categories. In the 'single term' category, we included statements that can only be categorized into one single theme. Often times, participants provide a statement which can be categorized into multiple themes. These statements contain meanings which overlap across multiple themes. Therefore, we grouped the statements into two or more categories under the 'in combination' category. We will only describe themes that more than 30 respondents referred to and we did not consider the statements from fewer than 30 respondents since these may be unrepresentative of the population. Participant's statements for their perception of health were coded with H (e.g., H1, H2, etc.) and their statements for their perception of sickness were coded with S (e.g., S12, $\mathrm{S} 13$, etc.). The results were ordered from the most to the least frequently mentioned theme.

\section{The meaning of health}

\section{Health as a Physical Condition}

About $23.8 \%$ of the respondents perceived their health solely in term of a physical condition (single-term category). Health was identified as the optimal functioning of the organs and the immune system, fitness, strength, and as being physically safe and sound. 
'Having a physical condition-including a good immune system-which can fight off disease and bacteria' (female, 18 years old)

'Having strength and a strong immune system which help us stay healthy. Health is related to the body organs (male, 22 years old)

Almost half of the responses indicated health as a physical state in combination with other condition. According to the respondents, health could be interpreted as a combination of the physical condition and the psychological condition [H1] being psychologically and physically OK. Psychologically: feeling no stress, not having many things on your mind, not being dominated by negative thinking. Physically: having a lot of energy, not easily getting ill, having passion and not being dull' (male, 20 years old); spiritual [H2] 'a physical condition which is free from pain or diseases, healthy both physically and spiritually' (female, 18 years old); social [H3] 'being physically free of pain or weaknesses, so one can interact socially and to easily adapt'(female, 18 years old,); and a capability to carry out daily activities [H4] 'when someone physically has the energy or stamina to carry out activities in a normal way' (female, 20 years old).

Table 1. Respondent Characteristics.

\begin{tabular}{|c|c|c|}
\hline Characteristic & $N$ & $(\%)$ \\
\hline \multicolumn{3}{|l|}{ Sex } \\
\hline Male & 313 & 48.91 \\
\hline Female & 327 & 51.09 \\
\hline \multicolumn{3}{|l|}{ Age } \\
\hline Mean (range) & 23.22 & $(12-60)$ \\
\hline $12-15$ years old & 8 & 1.3 \\
\hline 16-19 years old & 268 & 41.9 \\
\hline 20-30 years old & 318 & 49.7 \\
\hline $31-60$ years old & 46 & 7.2 \\
\hline \multicolumn{3}{|l|}{ Education } \\
\hline Elementary & 5 & 0.78 \\
\hline Junior High School & 24 & 3.75 \\
\hline Senior High School & 501 & 78.28 \\
\hline Diploma & 13 & 2.03 \\
\hline College Graduate & 91 & 14.22 \\
\hline Masters & 6 & 0.94 \\
\hline \multicolumn{3}{|c|}{ Income/ allowance per month (IDR) } \\
\hline$<1$ million & 273 & 42.65 \\
\hline 1-3 million & 242 & 37.81 \\
\hline$>3$ million & 125 & 19.54 \\
\hline
\end{tabular}


Table 2. Themes of the Meaning of Health.

\begin{tabular}{|c|c|c|c|c|c|}
\hline \multirow{2}{*}{$\begin{array}{l}\text { Main Themes } \\
\text { Single-Term }\end{array}$} & & \multirow{2}{*}{$\%$} & \multirow{2}{*}{$\begin{array}{l}\text { Subcategories } \\
\text { In Combination }\end{array}$} & \multirow{2}{*}{$N$} & \multirow{2}{*}{$\%$} \\
\hline & & & & & \\
\hline Physical & 152 & 23.8 & $\begin{array}{l}\text { Physical and Psychological } \\
\text { Physical and Spiritual } \\
\text { Physical and Capability to carry out daily activities } \\
\text { Physical, Psychological, and Spiritual } \\
\text { Physical, Spiritual, and Capability to carry out daily } \\
\text { activities } \\
\text { Physical, Psychological, and Social } \\
\text { Physical, Psychological, and Capability to carry out daily } \\
\text { activities } \\
\text { Physical, Psychological, Spiritual, Social, and Economic } \\
\text { Physical, Psychological, and Healthy Lifestyle } \\
\text { Physical and Social } \\
\text { Absence of physical, psychological, and economic } \\
\text { problems }\end{array}$ & $\begin{array}{l}99 \\
71 \\
44 \\
31 \\
15\end{array}$ & $\begin{array}{l}15.5 \\
11.1 \\
6.9 \\
4.8 \\
2.3\end{array}$ \\
\hline Psychological & 64 & 10 & $\begin{array}{l}\text { Physical and Psychological } \\
\text { Physical, Psychological, and Spiritual } \\
\text { Physical, Psychological, and Social } \\
\text { Physical, Psychological, and Capability to carry out daily } \\
\text { activities } \\
\text { Physical, Psychological, Spiritual, Social, and Economic } \\
\text { Psychological and Capability to carry out daily activities } \\
\text { Physical, Psychological, and Healthy Lifestyle } \\
\text { Absence of physical, psychological, and economic } \\
\text { problems }\end{array}$ & $\begin{array}{l}99 \\
31 \\
13 \\
13\end{array}$ & $\begin{array}{l}1.7 \\
1.6 \\
0.6 \\
0.3\end{array}$ \\
\hline Spiritual & 15 & 2.3 & $\begin{array}{l}\text { Physical and Spiritual } \\
\text { Physical, Psychological, and Spiritual } \\
\text { Physical, Spiritual, and Capability to do daily activities } \\
\text { Physical, Psychological, Spiritual, Social, and Economic }\end{array}$ & $\begin{array}{l}71 \\
31 \\
15 \\
11\end{array}$ & $\begin{array}{l}11.1 \\
4.8 \\
2.3 \\
1.7\end{array}$ \\
\hline $\begin{array}{l}\text { Capability to } \\
\text { carry out daily } \\
\text { activities }\end{array}$ & 50 & 7.8 & $\begin{array}{l}\text { Physical and Capability to carry out daily activities } \\
\text { Physical, Spiritual, and Capability to carry out daily } \\
\text { activities } \\
\text { Physical, Psychological, and Capability to carry out daily } \\
\text { activities }\end{array}$ & $\begin{array}{l}44 \\
15\end{array}$ & $\begin{array}{l}6.9 \\
2.3\end{array}$ \\
\hline $\begin{array}{l}\text { Healthy } \\
\text { lifestyles }\end{array}$ & 35 & 5.5 & $\begin{array}{l}\text { Psychological and Capability to carry out daily } \\
\text { Physical, Psychological, and Healthy Lifestyle }\end{array}$ & 4 & 0.6 \\
\hline Economic * & 8 & 1.3 & $\begin{array}{l}\text { Absence of physical, psychological, and economic } \\
\text { problems }\end{array}$ & 2 & 1.3 \\
\hline
\end{tabular}

* See Appendix D for sentence examples. 
Table 3. Themes Regarding the Meaning of Sickness.

\begin{tabular}{|c|c|c|c|c|c|}
\hline \multirow{2}{*}{$\begin{array}{l}\text { Main Themes } \\
\text { Single-Term }\end{array}$} & \multirow{2}{*}{$N$} & \multirow{2}{*}{$\%$} & \multirow{2}{*}{$\begin{array}{l}\text { Subcategories } \\
\text { In Combination }\end{array}$} & \multirow{2}{*}{$N$} & \multirow{2}{*}{$\%$} \\
\hline & & & & & \\
\hline \multirow{13}{*}{ Physical } & \multirow{13}{*}{168} & \multirow{13}{*}{26.3} & Physical and Psychological & & \\
\hline & & & Physical and Inability to carry out daily & 100 & 15.6 \\
\hline & & & activities & 59 & 9.2 \\
\hline & & & Physical and Spiritual & 41 & 6.4 \\
\hline & & & Physical, Psychological, and Spiritual & 21 & 3.3 \\
\hline & & & Physical, Psychological, and Inability to carry & 13 & 2.0 \\
\hline & & & out daily activities & & \\
\hline & & & Physical, Psychological, Spiritual, Social, & 12 & 1.9 \\
\hline & & & Economic, and Inability to carry out daily & & \\
\hline & & & activities & 10 & 1.6 \\
\hline & & & Physical, Psychological, and Social & 7 & 1.1 \\
\hline & & & Physical and Unhealthy Lifestyle & 2 & 0.3 \\
\hline & & & Physical and Social & & \\
\hline \multirow{7}{*}{ Psychological } & \multirow{7}{*}{43} & \multirow{7}{*}{6.7} & \multirow{7}{*}{$\begin{array}{l}\text { Physical and Psychological } \\
\text { Physical, Psychological, and Spiritual } \\
\text { Psychological and Activities } \\
\text { Physical, Psychological, and Inability to carry } \\
\text { out daily activities } \\
\text { Physical, Psychological, Spiritual, Social, } \\
\text { Economic, and Inability to carry out daily } \\
\text { activities } \\
\text { Physical, Psychological, and Social }\end{array}$} & 100 & \\
\hline & & & & 100 & 15.6 \\
\hline & & & & 21 & 3.3 \\
\hline & & & & 15 & 2.3 \\
\hline & & & & 13 & 2.0 \\
\hline & & & & 12 & 1.9 \\
\hline & & & & 10 & 1.6 \\
\hline $\begin{array}{l}\text { Abnormal circumstances } \\
\text { and bad situations }\end{array}$ & 62 & 9.7 & & & \\
\hline \multirow{4}{*}{ Spiritual } & \multirow{4}{*}{12} & \multirow{4}{*}{1.9} & Physical and Spiritual & & \\
\hline & & & Physical, Psychological, and Spiritual & 41 & 6.4 \\
\hline & & & Physical, Psychological, Spiritual, Social, & 21 & 3.3 \\
\hline & & & $\begin{array}{l}\text { Economic, and Inability to carry out daily } \\
\text { activities }\end{array}$ & 12 & 1.9 \\
\hline \multirow{4}{*}{$\begin{array}{l}\text { Inability to carry out } \\
\text { daily activities }\end{array}$} & \multirow{4}{*}{45} & \multirow{4}{*}{7.0} & \multirow{4}{*}{$\begin{array}{l}\text { Physical and Inability to carry out daily } \\
\text { activities } \\
\text { Psychological and Activities } \\
\text { Physical, Psychological, and Inability to carry } \\
\text { out daily activities } \\
\text { Physical, Psychological, Spiritual, Social, } \\
\text { Economic, and Inability to carry out daily } \\
\text { activities }\end{array}$} & 59 & 9.2 \\
\hline & & & & 15 & 2.3 \\
\hline & & & & $\begin{array}{l}15 \\
13\end{array}$ & $\begin{array}{l}2.3 \\
2.0\end{array}$ \\
\hline & & & & 12 & 1.9 \\
\hline Unhealthy Lifestyle ** & 21 & 3.3 & Physical and Unhealthy Lifestyle & 7 & 1.1 \\
\hline Economic $* * *$ & 9 & 1.4 & $\begin{array}{l}\text { Physical, Psychological, Spiritual, Social, } \\
\text { Economic, and Inability to carry out daily } \\
\text { activities }\end{array}$ & 12 & 1.9 \\
\hline
\end{tabular}

** See Appendix E for sentence examples; *** See Appendix F for sentence examples. 
In addition to perceiving health as a combination of the physical condition with one other condition, some respondents conceptualized health as a combination of physical condition with more than one condition-for example, they perceived health as a combination of physical condition, psychological condition and having a healthy lifestyle [H5] '[health is] about a good physical and spiritual condition because one has a regular lifestyle and discipline' (male, 22 years old); or the combination of many different conditions [H6] 'being psychologically, mentally, physically and socially healthy, so that daily activities are not disrupted' (female, 18 years old); as well as the absence of physical, psychological, and economic disorders [H7] 'a condition in which someone does not experience any physical, psychological, or economical disorder' (male, 19 years old).

[H8] 'A state in which the body and the soul are in a normal condition, and there are no limitations on performing activities' (male, 30 years old)

[H9] 'Free from pain and physical weaknesses. When you can socialize, adapt, and have a good personality and state of mind' (male, 18 years old)

[H10] 'A condition when we feel happiness both physically and spiritually; and to feel comfortable to do anything, without any interference from the inside [e.g., physical constraints]' (female, 19 years old)

\section{Health as a Psychological Condition}

Respondents perceived health as a psychological condition, which was perceived as a combination of calmness, peace, and joy in heart, mind, and soul - even in attitude. It was expressed as a pleasant and soothing feeling and could be interpreted as a clear and unencumbered state of mind.

'Whenever we feel jubilant, comfortable and safe, as well as in a fresh condition' (female, 23 years old)

'Live in peace and unencumbered by problems (female, 25 years old)'

Slightly fewer than a third of the respondents considered health as a psychological condition that was related to one other condition: physical (see H1; H5; H8; H9), spiritual (see H6), social (see H9), the ability to carry out daily activities (see H8), and a healthy lifestyle (see H5). The psychological condition was also included in combinations of many conditions (see H6), e.g., health as the absence of physical, psychological, and economic disorder (see H7). In addition, some respondents stressed the combination of psychological conditions and the ability to perform activities. 
'Can perform various activities with a rational mind' (male, 26 years old)

[H11] 'Comfortable, peaceful, stable condition so that someone can carry out daily activities well' (male, 24 years old)

\section{Health as a Spiritual Condition}

Respondents also defined health as a reference to their spiritual values. Health was identified as a grace and a blessing. Some respondents pointed out that health cannot be replaced by anything, and it was seen as a blessing to be continually grateful for.

'Something that cannot be replaced by anything and something that we should be continually grateful for, because being healthy is a blessing in life'(male, 29 years old)

Health was also described as a combination of spirituality with one or more other condition, such as the physical condition (see H2; H10), the psychological condition (see H6), the ability to carry out activities (see H10), or a combination these conditions (see H6).

\section{Health as a Capability to Carry out Daily Activities}

Fifty respondents $(7.8 \%)$ perceived health in terms of the ability to carry out activities without being distracted.

'Being able to do something with [full] capacity and to the best of one's abilities' (female, 23 years old)

This theme was also mentioned in combination with other conditions, such as physical (see H4; H8; H10), spiritual (see H10), and psychological (see H11) conditions.

\section{Health as a Healthy Lifestyle}

A lifestyle was perceived to be healthy in cases in which it includes a healthy diet, an exercise, and a clean environment.

'[To] maintain cleanliness, [to] exercise, [to] eat regularly and drink plenty of water' (male, 26 years old)

'The key to being healthy is hygiene' (female, 40 years old)

The respondents also reported that health was perceived as a combination of physical and psychological components, as well as a healthy lifestyle (see H5). 


\section{The Meaning of Sickness \\ Sickness as a Physical Condition}

About $26.3 \%$ of the respondents perceived sickness as a physical condition. Sickness was defined as a weak physical condition or the non-functioning of certain body parts due to a decrease in the immune system because of the presence of a disease or virus.

\footnotetext{
'Weak body and [to feel] unfit or drastically decreased-stamina' (female, 18 years old)
}

'[The] condition of disorder or dysfunction of the body or a body part' (male, 32 years old)

Furthermore, two-fifths of the respondents perceived their own sickness in combination of a physical condition and other conditions, including psychological [S12] the condition of a physical, mental or disturbed mind or having a (psychological) disability" (male, 25 years old); spiritual [S13] 'physically, when we are injured. Inwardly, when we have a lot of thoughts and cannot find a way out' (female, 25 years old); social [S14] 'one's condition when one is exposed to disease and disability, both physically and socially' (female, 20 years old); an inability to carry out daily activities [S15] 'the circumstances in which the body is not in a good or prime condition because of a disease or germs from a particular virus, so that the body becomes weak and this hampers everyday activities' (female, 21 years old); and an unhealthy lifestyle [S16] 'the state of the body affected by disease and followed by a dirty and nasty environment' (male, 19 years old).

Some respondents also conceptualized sickness as physical condition combined with two or more other conditions of sickness at the same time, for example, a combination of physical, psychological, and spiritual conditions [S17] 'someone who does not have a passion for life. Health or sickness is not always because of a physical factor, because to be human [one] has [the] elements of [the] body, [the] soul and [the] spirit. Then, if one of these [elements] goes wrong, a person will become sick' (female, 23 years old); or physical, psychological, and an inability to carry out daily activities [S18] 'there are parts of the body that are ill or disturbed or in a bad condition, [one] cannot move freely or think; the [psychological] heart is often unhappy or stressed' (female, 57 years old); physical, psychological, and social [S19] '[the] circumstances when someone needs others to help cure the illness [physical and mental]' (male, 18 years old); and a combination of many conditions [S20] 'the circumstances in which a person experiences a physical disorder, or psychological or economic problems, or all of these' (male, 19 years old).

\section{Sickness as a Psychological Condition}

Sickness was also seen as a psychological condition. Respondents expressed the notion that someone could become sick whenever one experiences stressful situations, such 
as unsolved problems, or some limitations on being able to exert one's free will. These stressful situations can cause discomfort and sad feelings, as well as loss of the spirit to live.

'Sick and healthy people are actually controlled by the mind, a healthy person will feel ill if his mind considers himself sick' (male, 19 years old)

More than a quarter of the respondents interpreted their sickness as a psychological condition in relation to other conditions such as a physical condition (see S12), a spiritual condition (see S17), and an inability to carry out daily activities (see S18). Respondents also conceptualized sickness as a combination of a psychological condition and an inability to perform activities.

[S21] 'Uncomfortable or unusual conditions so that the person feels uncomfortable or has problems in performing daily activities' (male, 24 years old)

[S22] 'Cannot think properly and cannot live a good life' (female, 20 years old)

\section{Sickness as an Abnormal Circumstances and Bad Situations}

Respondents regarded this particular theme as a single-term category; they did not perceive sickness as a combination of abnormal and bad situations with other situations or conditions.

'Abnormal, out of the ordinary conditions, below the normal line' (male, 39 years old)

\section{Sickness as a Spiritual Condition}

Some respondents described sickness as a temptation and a test, as someone staying away from God. From this point of view, a sickness exists in order to keep humans close to God.

'One result of human negligence and one of the means of allowing humans to be closer to God' (female, 32 years old)

Respondents also identified sickness as a combination of a spiritual condition with other conditions, such as physical (see S13) and psychological (see S17).

\section{Sickness as an Inability to Carry out Daily Activities}

Respondents stressed the meaning of sickness as a person's inability to perform activities. The inconvenience related to the inability to carry out daily activities was also interpreted as sickness. Although this was seen as a natural part of life, sickness 
was also understood as the inability to achieve maximum results when one is working on something.

'Cannot do [any] activities' (female, 28 years old)

'When I cannot achieve the maximum in every way' (male, 19 years old)

Sickness was also seen as an inability to carry out daily activities in relation to both physical (see S15, S18) and psychological conditions (see S18, S21; S22).

\section{Discussion}

The aim of the present study was to explore the perceptions of health and sickness among Jakartans. A more comprehensive understanding of health and sickness will shed light on the cultural context of health and sickness that influence certain actions, such as whether or not one would adopt a healthy behavior or a healthy lifestyle. This information can help health care providers to provide services which are in line with the public understanding of health and sickness. Overall, our findings demonstrate that respondents perceived health and sickness not only as a physical condition, but also in a combination of many other conditions. Simultaneously, we also found that some respondents identified their health and sickness in one-single condition unconnected to other conditions of health or sickness (see appendix A).

In this study, health was seen as a positive term. Health was perceived in terms of fitness and bodily strength (physical), the calmness of the mind and the soul (psychological), a close relationship with God (spiritual), and the ability to be active or productive (capability to carry out daily activities). Some respondents described a healthy condition as a result of a healthy lifestyle which includes the discipline in regards to maintain a healthy diet and to carry out a physical activity. Other respondents perceived health as a condition that allowed people to do multiple activities. These findings were in line with a previous study from Hidajat (Hidajat, 2005) in East Java, Jogjakarta, and Bali regions which showed that people from East Java and Jogjakarta perceived their health and sickness from Javanese philosophy and attitude of life. In the Balinese society, for instance, health and sickness were related to the opportunity to pour out one's art expression and self-improvement values. Our results were also similar to those of previous research in which health has been interpreted as a positive attribute-or example, as being well-functioning, having a good physical condition, a positive state of well-being, a positive feeling, and an ability to carry out desired or required activities (Levesque, Li, \& Bohémier, 2013).

Our result showed that respondents perceived health as an optimal physical condition. This physical condition of health was also linked to other conditions, such as psychological and spiritual conditions of health, the capability to carry out daily 
activities, and to have a healthy lifestyle. These conditions were mentioned in isolation or as having a causal effect on another condition. For example, health was interpreted as a healthy bodily condition that enables someone to perform various daily activities. So, for this example, healthy conditions cannot be interpreted as only a physical condition, but also as the ability to perform daily activities. This result was similar to findings from previous studies about health, which either investigated the meaning of health from the perception of the general population or some specific subgroup (Fijal \& Beagan, 2019; Isaak \& Marchessault, 2008; Mendelson, 2002; Witmer, Bocarro, \& Henderson, 2011). For instance, Mendelson (Mendelson, 2002) showed that Mexican American women perceived health as a compilation of a good physical health, a sound mental health, and a socially and spiritually satisfying life. The study of Isaak \& Marchessault (Isaak \& Marchessault, 2008) on youth and adults of an Aboriginal community in a Northern Manitoba First Nations Community also defined health as various combinations of: changes in diet and activity, positive adult role models, traditional practices, and the significance of making good choices.

Various studies have stressed the finding that health can be both directly and indirectly influenced by social, economic and environmental circumstances (Graham \& White, 2016; Pickett \& Wilkinson, 2015; Whitehead et al., 2016). This perception was also reflected in the daily activities of Jakartans, who associated being 'healthy' with the ability to work, to perform daily activities, and to be productive. This finding might be related to the fact that most of the respondents of the present study were from the low-income group (below IDR three million per month), which is close to $65 \%$ of the Jakartans who works as laborers and who receive daily (as opposed to monthly) payments (Statistics Indonesia, 2016). Based on their work hours in February 2016, 4.5 million people $(89.83 \%)$ in Jakarta worked for 35 hours or more per week. Receiving daily payments also mean that whenever laborers take a sick leave, they do not get their daily wage (Simone, 2015). This leads them to worry about the income reduction due to the unpaid sick leave and thus, consequently, made them to still work in spite of being sick. This situation is different to that in the Western countries, where laws that require a company to pay sick leave exist, for example, up to five days for employees who need to recover from the flu (Enneman, 2014; Jongepier, 2017; Ministerie van Sociale Zaken en Werkgelegenheid, 2011).

A healthy lifestyle was identified as one condition of health by the respondents. Nowadays, in Jakarta, many people go to the gym, or take yoga, Zumba or Pilates classes (Arthen, 2016). At the same time, however, as long as they work out or go to the gym, people are less likely to care about what they eat, because they believe that they can eat whatever they want (Dhorothea, 2016). In contrast to this view, the results of the present study showed that respondents tended to emphasize a healthy/ unhealthy diet, and only a few of the respondents stressed the importance of exercise as part of health and sickness. This suggests that our respondents do not view health and having a healthy lifestyle in a comprehensive way. This limited perception of health may contribute to the high prevalence of cardiovascular disease, obesity and diabetes in Jakarta (WHO 
Country Office Indonesia, 2016). These diseases are mainly caused by an unhealthy lifestyle, particularly an unhealthy diet and a lack of exercise (Arthen, 2016).

Importantly, health was also conceptualized in terms of the peace of mind or the psychological tranquility by our respondents. The psychological condition was described as a pleasant and soothing feeling, and as a clear and unencumbered state of mind. This perception may relate to the needs of the Jakartans for psychological care and recreational areas. Most leisure spots in Jakarta are in shopping malls, due to the lack of public space despite the government's effort to build more urban open spaces (Damarjati, 2016). Residents usually travel outside Jakarta, for example to Puncak, Bogor or Bandung area for recreational activities; however, they must navigate heavy traffics to reach these destinations (Dewi, 2016).

The high demands and the fast-paced lifestyle in Jakarta are believed to contribute to high levels of stress in the community (Prathivi, 2015). Nevertheless, psychological health services at the primary health level are limited, due to the low ratio of clinical psychologists compared to the Indonesian population (Ika, 2015). Reports indicate that Indonesia has 500-600 clinical psychologists, whereas, Jakarta alone needs 341 clinical psychologists to meet the demands of the primary health services. This means that, only a few primary health services in Jakarta have a clinical psychologist as part of their team (Herlinda, 2015). This is in stark contrast to psychological services provided in most primary health services in Jogjakarta and Sleman (Ika, 2015; Sari, 2016). Clinical psychologists at primary health services in Jogjakarta provide psychological services on every working-day at a low cost ( \pm USD 0.5$)$ due to the financial supports from the government (Martono, 2011). The availability of these services makes people more familiar with psychologists, more comfortable with the idea of consulting with them, and it reduces any negative stigma on psychological services (Pramudiarja, 2014).

While health was associated with positive terms in our study, our respondents perceive sickness in negative terms, such as weakness, inability, being infected with disease, feeling uncomfortable, and being cursed. Sickness was not interpreted simply as non-healthy conditions; instead, this conceptualization of sickness can be seen as more complex than the biomedical health approach (Conti, 2018), in which health and sickness are mostly seen as a result of, for example, sanitation and infection (Kahissay et al., 2017). Here, sickness was defined as abnormal conditions and bad situations, when the result of things not happening as they should, or as something out of the ordinary.

Sickness, for the respondents, was associated with spirituality, for example. It was perceived to be trials by God and a test of one's faith. Respondents tended to believe that sickness was a lesson taught from God to make them appreciate the life that had been freely given to them. People thus use prayers in the hope of healing their sickness, because there is a tendency to see everything spiritually and put it in God's hands for better results. This finding can be linked to research carried out in other countries which has investigated sickness as a reference to various conditions of spirituality. For example, the importance of praying, reading the Bible, attending church, or practicing traditional activities to remain in harmony with cultural values (Mendelson, 2002) 
In a society where sickness is interpreted from a spiritual perspective, sickness also can be treated differently (as compared to the biomedical approach) (Conti, 2018; Richter, 2003). People tend to go to traditional health practitioners, where they drink herbal regimens such as Indonesian herbal drinks (jamu) or Chinese herbs, or receive acupuncture and massages to heal their sicknesses (McKay \& Wangchuk, 2018; Nurhayati \& Widowati, 2017; Qiu, 2007). Peltzer \& Machleidt (Peltzer \& Machleidt, 1992) found that people in developing-countries seek traditional medicines because they believe in the effectiveness of these medicines, sometimes in spite of the lack of evidence for their benefits. The high confidence they place in traditional medicines often means the people would try them first before they go to a physician (Nurhayati \& Widowati, 2017).

Respondents in our study implied that there were sicknesses which result in the inability to get up or carry out daily activities. This means that someone is called sick if one is not productive or cannot perform daily activities. In this perspective, sickness cannot be equated with a physical condition because people tend to work despite being (clinically/physically) sick. The tendency to work despite being sick can also be linked to the spread of diseases as well as being related to income issues, as explained before. If someone still goes to work while being sick, there is a possibility that others will be infected, especially in a highly-populated city (Heesterbeek et al., 2015; Sadilek, Kautz, \& Silenzio, 2012) such as Jakarta.

Our results have several implications. Firstly, there is a need to improve the health education and promotion systems to adapt to the fast-paced life in Jakarta. This information needs to catch the Jakartans' attention and at the same time motivate them to implement it. The health education system can include the importance of a healthy lifestyle, including exercising alongside having a healthy diet. The information about how diseases spread should also be disclosed, so people would understand the risk of going to work despite being sick. Health promotion can be done by providing leisure spots in the city, for example, a playground with sports facilities, so that people may get to relax while they exercise. Secondly, the government needs to consider involving religious leaders in conducting health promotions. This suggestion arises from the fact that people perceive health and sickness from a religious perspective. The involvement of religious leaders might contribute to fulfilling the religious aspect of health, without putting aside the community's health in general. Thirdly, the government needs to consider providing health care that facilitates typical laborers to get health checks. For example, to provide services after normal working hours and at the weekends. This suggestion might counter the financial issues for people who are paid on a daily basis. Lastly, given the finding that people tend to visit traditional health practitioners, the government should consider including traditional, complementary, and alternative medicine (or TCAM) in its health promotion and health intervention activities. A collaboration between modern medicine and evidence-based traditional or alternative medicines may help to provide a better health care service, one that is more in line with people's needs, beliefs, and values. It could also be used to maintain and improve the health of particular communities. 
Despite the study's contribution, this research had several limitations. Firstly, the research findings may not be generalizable to other populations in Indonesia. As stated before, the cultural context influences people's perceptions and experience. As Indonesia is a country with a high cultural diversity, each region in Indonesia has its own cultural characteristics. Additionally, to generalize our results to the Jakarta population better, it is necessary to investigate the perceptions of health and sickness of a larger sample of Jakartans with quantitative and statistical procedures. Secondly, as stated at the results section, the research assistant's age might have an influence on the responsiveness of the prospective respondents (i.e., respondents with the similar age range with those of the assistants might be more inclined to participate in the study). Further research might consider recruiting research assistants with more diverse characteristics.

\section{Conclusions}

This study discovered that Jakartans perception about health can be grouped into five main categories: health as a physical condition, health as a psychological condition, health as a spiritual condition, health as a capability to carry out daily activities, and health as a healthy lifestyle. Meanwhile, sickness is perceived as a physical condition, a psychological condition, an abnormal circumstance and bad situations, a spiritual condition, and an inability to carry out daily activities. This multidimensional view has effects on Jakartans behavior towards health and sickness. For instance, this view may explain why some people keep working despite of being sick, and why some people use prayers in the hope of healing their sickness. Additionally, we also discussed that the current health system in Jakarta, which emphasizes on physical conditions and is heavily influenced by the biomedical perspective, may not accommodate this multidimensional view of health and sickness. 
2 


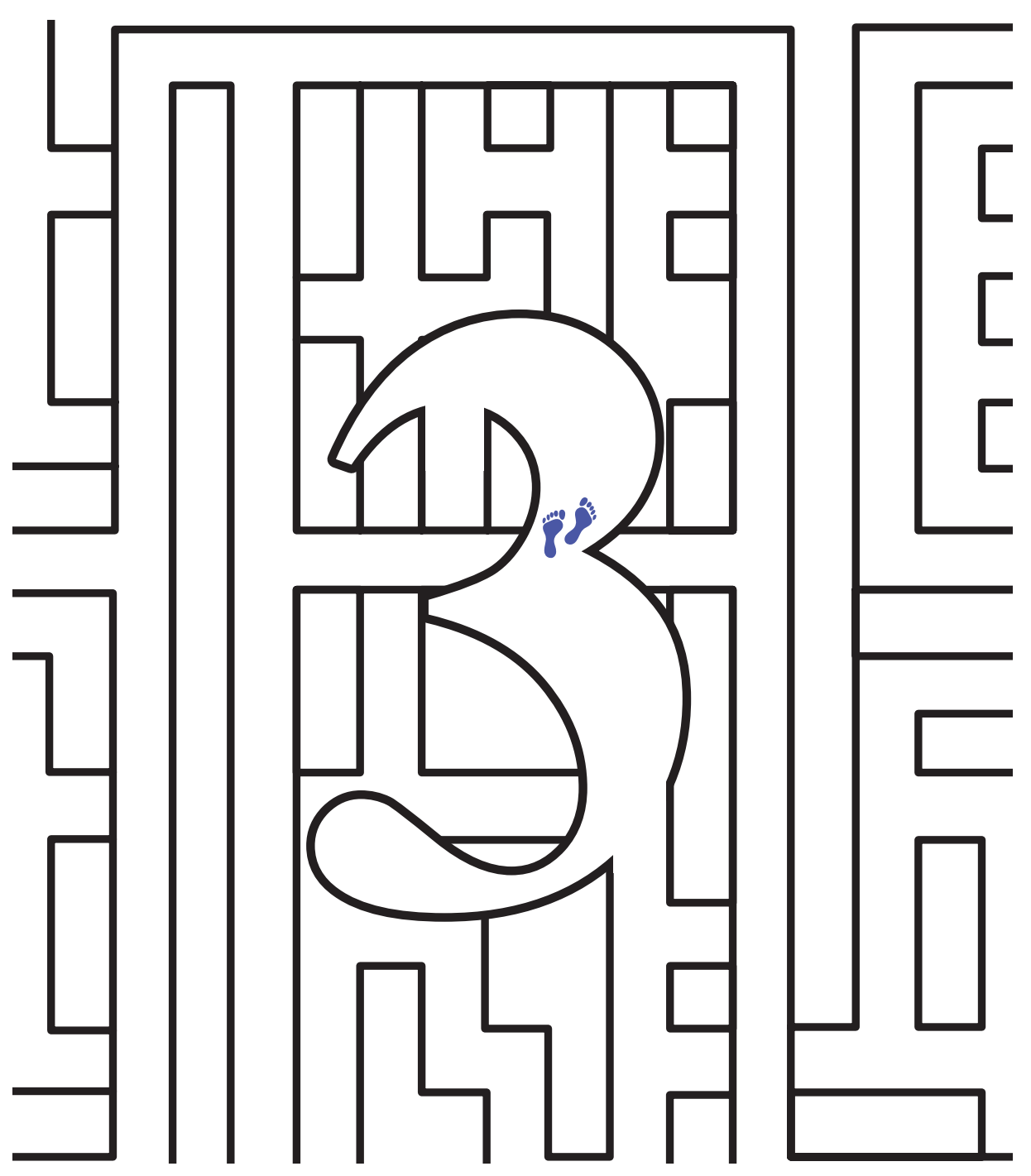




\section{Chapter 3.}

\section{Jakartans' Perceptions of Health Care Services}

Based on:

Sokang, Y. A., Westmaas, A. H., \& Kok, G. (2019). Jakartans' Perceptions of Health Care Services. Frontiers in Public Health, 7(September), 1-8. https://doi.org/10.3389/ fpubh.2019.00277 


\begin{abstract}
Health Care Services (HCSs) should implement ongoing innovation and continuously improve their quality. However, in evaluating the quality of HCSs, too little attention has been given to the experience of the users concerning the acquired services. This study focused on how the community values the current services in order to improve HCSs in Indonesia, especially in Jakarta. Four focus group discussions were conducted among 45 community members in the Grogol Petamburan sub-district, in Jakarta. Participants were recruited using a convenience sampling and the data were analyzed using a combination of human coding and NVivo-12. Overall, we found that participants had a negative view of the government-mandated Community Health Centers (CHCs) and they preferred to visit private clinics and hospitals over the CHCs. Participants associated CHCs with unfriendly staff, longer waiting times, shorter opening hours, and crowded visitors. At the same time, participants had a positive view on the affordability of the CHCs. Additionally, we found the reasoning of Jakartans' (i.e., the citizens of Jakarta city) on using self- and traditional treatments before visiting HCSs and they also expressed the need for psychological services at CHCs. The discussion focuses on the results as feedback on how the government and health care providers may facilitate the community's needs in providing HCSs in Indonesia, especially Jakarta. In brief, we recommend the policy-makers to improve the hospitality of the staff members and the quality of the medical equipment; also, to provide psychological services at CHCs. These efforts need to be done while paying more attention to the cultural aspects of medicinal uses.
\end{abstract}




\section{Introduction}

Improving health through improving health services and health promotion activities is currently one of the six priority programs of the Indonesian government (Ministry of Health of the Republic of Indonesia, 2015a; Suryowati, 2016). However, the government has encountered many obstacles in running their health-improvement programs, especially in Jakarta. Specifically, they have had problems with limited human resources, inadequate health facilities, unhealthy behavior of the citizens, the population density, and poverty (Jakarta Provincial Health Agencies, 2016; Ministry of Health of the Republic of Indonesia, 2015a). Together with the heterogeneous nature of the citizens of Jakarta, this situation creates a complex demand for health care providers (Ministry of Health of the Republic of Indonesia, 2015a). To overcome these obstacles, the Health Ministry periodically evaluates the quality of Health Care Services (HCSs) by conducting an accreditation (Ministry of Health Republic of Indonesia, 2014b). This accreditation emphasizes the development of the quality and performance of HCSs through continuous improvements of its management system; its service and programs delivery system; and its implementation of risk management.

The Health care system in Indonesia is a combination of public and private health providers. The public health provider is the Indonesian government; in this case, the Ministry of Health at the national level, provincial governments at the provincial level, and the district/municipality governments for district level. The private health providers manage by not-for-profit or for-profit providers, and individual doctors and midwives who have a private clinic. The financial support of health centers is the responsibility of the provider. The health care user can pay for health services using personal funds, or by using insurance. Currently the Indonesian government promotes the use of a universal health insurance. With the insurance, the user could get a cheaper or even free service by visiting Community Health Center (CHC; Indonesian: Pusat Kesehatan Masyarakat (Puskesmas))(Mahendradhata et al., 2017).

To support the health improvement program, one needs to conduct a thorough evaluation of the current state of the available HCSs. A more inclusive approach to said HCSs evaluation would include the perspectives of its users (Pramanik, 2016); this inclusion would help stakeholders to understand the health care needs, demands, and interest of the communities better (Duku, Nketiah-Amponsah, Janssens, \& Pradhan, 2018; Mitropoulos, Vasileiou, \& Mitropoulos, 2018). Here, the users are considered as experts (Sofaer \& Firminger, 2005).

As a first step to evaluate the perspectives of health care users as experts, we conducted a study to understand Jakartans' (i.e., the citizens of Jakarta city) perceptions regarding health and sickness (Sokang, Westmaas, \& Kok, 2019a). We found that the Jakartans not only see health as a physical state, but also as psychological, spiritual, and the ability to perform daily activities productively states. We also found the importance of investigating the perspectives of communities to fully understand their beliefs, values, and needs, in order to provide health care services that fit the demands 
of the communities. Our study suggests that incorporating the perspectives of users is an important key to improve the quality of HCSs.

In spite of the importance of understanding the perspectives of health care users, only little research has been done on the perceptions and expectations of health care services users in Indonesia, especially in Jakarta. In absence of local empirical findings, the Jakartan government and health care providers are more likely to incorporate the typical Western approaches on health programs, which emphasize the biomedical perspective, to improve the quality of HCSs in Jakarta. Furthermore, the aforementioned periodical accreditation does not take into account the users' experiences in evaluating the quality of HCSs. The lack of information on the experiences of the community of existing HCSs users makes it difficult, if not impossible for policy-makers to identify and satisfy the needs of the community.

Therefore, the main goal of the current study was to evaluate how the health care users in Jakarta perceive the quality of its HCSs. The perspectives of users would provide critical information which can be used to evaluate to what extent the available HCSs meet the expectations of its users. The resulting data could provide a recommendation for the government, health care providers, and health promoters in Jakarta to formulate health programs and policies which fits the needs of the local communities.

\section{Methods}

As a framework, this study used the Intervention Mapping (IM) approach which consists of six steps. The approach focuses on using theory and evidence as a solid basis on building a health program planning (Bartholomew Eldredge et al., 2016); thus provides clear guideline descriptions of the planning and designing, developing, implementing, and evaluating the program objectives (Dalum et al., 2012; van Empelen et al., 2003). In this current study, we used the first step of IM which emphasizes the importance of needs assessment to understand the target community, in terms of behavior and environmental determinant, including the characteristics of the community. Using this approach, we aimed to understand the health care users' perception on the quality of HCSs, while paying attention to culture and social aspects of the issue, including the needs of the community.

\section{Participants}

Data was collected in four focus group discussions (FGDs) which led by the first author in October 2017. In total, 45 Jakartans from various age (20-66y/o), from low and middle economic status (+ 35-320 USD/month), and from various educational levels (elementary school-bachelor degree) participated (18 males; 27 females). They were recruited using a convenience sampling and have given written informed consent to participate in the study. The participants were the member of the Grogol Petamburan sub-district, in West Jakarta Municipality, Special Capital Region of Jakarta. The Special 
Capital Region of Jakarta consists of five municipalities with the similar characteristics of the community member; as they come from various backgrounds. Of the participants, 30 were native Jakartans or had identified themselves as Jakartans, and 15 were from other areas in Indonesia. The participants have equal access to $\mathrm{CHC}$, (private) clinic, and hospital; although not all of them applied for national insurance. Despite the various demographic backgrounds, there were no systematic differences regarding our respondent's opinions on the quality of HCSs in Jakarta. Therefore, the results and discussion will focus on the participants' perspectives regardless of their demographics.

\section{Materials and Procedures}

Data were collected in FGDs to acknowledge people's experience, and to capture and explore people's ideas and perceptions on the topic at hand (Carey \& Asbury, 2016; Woodyatt, Finneran, \& Stephenson, 2016). The FGDs were conducted in the Indonesian language, in locations that were convenient to the respondents, and lasted for 68 minutes on average (min 53'-max 90').

Four research assistants (graduate bachelor students in psychology) were trained to answer any questions potential respondents might have during the FGDs, to observe the FGDs and make notes, and to conduct analysis of the data together with the first author. Prior to data collection, the assistants acquired an approval letter from the local village office to conduct the study. Upon receiving the approval letter, they came to different local communities in the Grogol Petamburan sub-district during the afternoon (when people usually gather in front of their houses), they asked people whether they would like to participate in the study, and they provided the opportunities for people to ask questions regarding the study. If potential participants agreed to participate in the study, the assistants provided several options for the FGD venue. Upon agreement, the assistants sent a formal invitation to the participants to participate in the FGD. In the invitation letter, we again explainedthe purpose of the FGD, the procedures of the FGD, the outline of the FGD questions, and the place and the duration of FGD. A statement of confidentiality letter was enclosed in the invitation letter. After participants had agreed to participate, they received the informed consent form. In the informed consent, there was information about the study purpose, and agreement of using video and voice recorder during the FGD. This is followed by the information regarding the members of the research team (name, faculty, university, and email address), and a questionnaire for participants to indicate their demographics such as their sex, age, education level, job, income/ allowance per month, and origin.

The FGDs were opened with a welcome, and an introduction round of the team members and the participants. The first author, who acted as a facilitator then described the general purpose of the meeting, the data confidentiality, and ensured that participants filled and signed the written informed consent form. Next, the facilitator explained the FGD procedure and established the ground rules of the FGD. Furthermore, the facilitator introduced the topic of discussion and started the discussion. The questions of FGD were presented as open-endedly as possible. The guideline questions were 'If you are sick, 
where would you go?'; 'What are your expectations when you went there?'; 'How do they treat you? Does it fit your expectation?'. Unclear ideas or opinions were clarified by open and probing questions, such as 'Can you explain that?'; 'Why?'; 'Can you give an example?'. Before closing the FGD, the facilitator summarized the discussion and distributed a short survey among the participants to ask whether the participants wanted to add something to the summary. Afterwards, the facilitator closed the discussion by thanking the participants for their contributions.

\section{Analysis}

The FGDs were recorded and transcribed verbatim. Due to the lack of information on Jakartans' perceptions of HCSs, a content analysis with an inductive approach was applied to create categories of the responses (Hussein et al., 2014; Mayring, 2014). A combination of human coding and NVivo-12 was used to analyze the data. Human coding was applied to the Indonesian language version of the data. Open coding and categorizations were performed by the research assistants under the supervision of the first author. The texts were read repeatedly by the assistants to identify meaningful words or sentences. Words and sentences with similar meaning were grouped under one category (Khamis \& Njau, 2016). The saturation of the data was achieved when there was no new category and theme emerged from the texts. This approach is also called the inductive thematic saturation (Saunders et al., 2018). Later, the transcriptions were translated into English and analyzed using NVivo-12 by the authors. This process was done to ensure both human coding and NVivo-12 analyses would fit with one another.

\section{Results}

Although, we describe participants' views toward HCSs on separate themes, it is important to keep in mind that these themes were not entirely independent from one to another. The themes portrayed the participants' experience in using HCSs in Jakarta. Participants claimed to visit Community Health Center (CHC; Indonesian: Pusat Kesehatan Masyarakat (Puskesmas)) and (private) clinic more often than hospital, so only few statements describe their experience in hospitals.

\section{Hospitality of the staff members}

The hospitality of the staff members is the most emerging theme. This theme includes the durations of waiting time (before service) and for medical examinations. There were two topics that concern participants the most. Firstly, they perceived CHC staff as 'less friendly' [female, 20y/o], 'cruel', 'rude' and made them 'feel distinct' [female, 29y/o]. Secondly, the waiting time in CHCs was long due to a large number of people who typically visit the $\mathrm{CHC}$ during its opening hours. One participant stated that whether she 'wanted or not, as long as (the CHC is) open' [female, 35y/o], she would wait to get her treatments. However, after the patients got treated, the general practitioners only 
asked brief questions and 'immediately gave the solution, gave medications' [female, 41y/o]. Participants believe that the general practitioner treated them hastily because 'time is expensive' [female, 41y/o] for the general practitioner. This experience created a negative perception about the CHC.

Compared to the CHCs, participants preferred to get medical treatments at private clinics when they got ill. They perceived staff members of the private clinics as more friendly and welcoming. Additionally, the queue was shorter; therefore, they can get examined immediately. Likewise, one participant stated that the private clinics and hospitals were the best because the medical information was 'more specific; any kinds of complaints were more heard', so she was 'more satisfied' [female, 37y/o].

\section{Quality of the services}

The quality of HCSs' services includes the quality of its facilities and medical devices. With regard to $\mathrm{CHCs}$, participants believed that 'at most the medicines were generic medicines; even patented medicines were not there' [male, 20y/o]. Generic medicines were perceived to be less effective because the patients think that he/she "Needed to come back, took longer to recover' [female, 41y/o]. Participants assumed that CHCs only provide medicines for minor illnesses, such as coughs, colds, and fever. Some participants viewed $\mathrm{CHC}$ in a negative way, because 'on average the CHC's equipment mostly was old; maybe because it's never replaced' [male, 20y/o]. On the other hand, the participants realized that it happened because the $\mathrm{CHCs}$ 'only relied on subsidies from the government' [male, 20y/o].

Meanwhile, participants were more confident with the quality of medicines at private clinics. One participant shared that 'in (the) CHC, (I) had treatment; (after) three days (I was) not getting better, (in spite of) the medicine was given. After I run (went) to the clinic, thank God, (with) this medicine, (I got) healed. So, I choose clinics' [male, 32y/o]. Participants willingly pay more, because they believe that the clinics put quality first by providing specialists. On the contrary, a lady who has a baby found '(the vaccination) from the CHC likely to be trustable; it comes directly from the Minister of Health, from Health Office. The truth is, the clinics are the one that we are really wary of ... We do not know where their vaccine from, where it takes from' [female, 35y/o]. As a side note, this statement might be biased by news about bootleg-vaccines that came out prior to the FGDs.

\section{Service fee}

Participants had a positive view on CHCs concerning their service fee. The inexpensive cost for medicines and treatment made participants go to the CHC because 'if (I use) national health insurance (it's) free, even if (I) pay, only three thousand five thousand $(I D R ; \pm 0.21$ USD)' [female, 41y/o]. To receive a better treatment at larger hospitals, participants would ' $\mathrm{go}$ to the CHC to only ask for a referral letter; that's why I always go to the $C H C^{\prime}$ [male, $21 \mathrm{y} / \mathrm{o}$ ].

A more negative view was expressed towards the private clinics and hospitals' service fee. Most participants agreed that 'if (I) go to the clinic, (you) pay a little 
more expensive' [male, 46y/o], because 'it will cost a bit different from the CHC; the medicine's quality is definitely different' [male, 46y/o]. They went to the hospital only if they got a referral letter from the $\mathrm{CHC}$, so that the treatment will be free or cheaper than the regular treatment without a referral letter. One participant mentioned that her brother is a general practitioner in a hospital 'so, if my family gets a severe illness, directly to his hospital; my brother makes the referral (letter)' [female, 21y/o]. Nevertheless, a lady who has a baby disclosed that even though the hospital is expensive, she will use the service again if her child is sick.

\section{Opening hours and distance}

Participants tend to have a negative perception of CHCs due to the short opening hours. Moreover, the long distances making them less motivated to visit the $\mathrm{CHC}$ as long distances made them need extra time and funds.

In contrast, the private clinics are often open for 24 hours, and they can be relatively close. The availability and distance allowed participants to 'go to the clinic because the clinic is 24 hours. Once, around one o'clock or two AM (I was) sick and went straight there (the clinic)' [female, 21y/o]. They were able to visit the clinic on a walking distance, so they did not need extra money. Private hospitals are also generally open for 24 hours. However, '(it's) tiring to go to the hospital, (because it's) far, (and) moreover (it's) expensive' [female, $42 \mathrm{y} / \mathrm{o}]$.

\section{Self- and traditional treatments}

In addition to their views on HCSs, most participants also indicated that they preferred to do self-treatment before going to the HCSs when they were sick. One participant stated 'If sick, (I) usually (buy) medicine (from small) stall first; later, if still sick after few days, (I) go to the CHC [male, 36y/o]. Participants indicated that they would take a rest at home and ignore their pain while they observed how their sickness develops. If their condition improves, they would not seek additional treatments. They will visit a general practitioner if their illness got worse.

Some participants stated that they prefer the traditional medicine such as herbal drinks (Indonesian: jamu) and they applied the local practice of coining (Indonesian: kerokan). Participants choose to do self-treatments in cases in which their sickness is a minor one, for example when they got a cold (Indonesian: masuk angin). In this case, there was no need for a special treatment such as going to the general practitioner. One participant said 'if I get sick ... mostly buy medicine or look for traditional medicine. Because traditional medicine, it is herbal and it is passed down, given to us. From our grandfather's era the medicine is the same. Indeed, we already know it's more powerful than the medicines we purchase in pharmacies with whatever substances in it. I prefer traditional medicine which (is) definitely herbal; it's an inheritance from long time ago' [male, 22y/o]. 


\section{The need for psychological services}

Lastly, participants expressed their need for psychological services at HCSs. They mentioned the need for counseling for their daily life problems, for child development issues, and to get additional information about parenting skills, such as on guiding their children to learn more efficiently and to deal with teenagers.

In one of the communities, there were residents with severe mental disorders. The participants indicated that they had proposed to bring the patient to mental institution, but the family of the patient declined the idea. The participants chose to be quiet in this case, because they have limited knowledge about mental health and were worried that they would offend the family. One participant stated 'the psychologists (are) special(ist), (they know) what to ask to the patient. So, (they) already know, oh I want to ask this and this, can (do/say) this. For us, sometimes (when we) ask the person get angry, we will stop' [female, 57y/o].

\section{Discussion}

The aim of the present study was to understand the perceptions of Jakartans in regard to the quality of available HCSs. A better understanding of Jakartans' perceptions of HCSs would contribute to the evaluation of to what extent the available HCSs meet the expectations of the users. This information can help the government, health care providers, and health promoters to formulate health policies and programs for Jakartans while taking the community's point of view into account. Overall, our findings indicate that the HCSs, currently available in Jakarta, do not provide services that meet the values that are considered important by the communities. This leads to the tendency of the participants to do self-treatment before going to the health care centers.

As stated before, we discussed the themes separately, however, the readers may notice that they were not entirely independent from one to another. The hospitality of the staff members is the most expressed theme by our participants in relation to the perception of HCSs in Jakarta. This theme includes the length of waiting time and for medical examination. At the same time, the waiting time and the duration of medical examination links with opening hours and distance, and with the quality of the facilities and medical devices.

Previous studies have found that the hospitality of health care services staff members is important to shape a positive image for the users (Kayral, 2014). Users, in this case the patient and her/his family, prefer friendly staff because they are approachable and easy to make contact with. Users perceived these characteristics as sympathetic and caring; so that, they trust the staff members to take care of them or their family member (European Patients' Forum, 2017). Caring and friendly services have also been found to be associated with the increase of user satisfaction (Mitropoulos et al., 2018; Senitan, Alhaiti, \& Gillespie, 2018). As a result, the intention to visit the same HCSs and to use the services of HCSs will increase (Tamanam, 2016). In line with these findings, our 
participants prioritized hospitality over service fee; they are willing to pay more as long as they get friendly services from the staff. In our case, the participants chose private clinics over CHCs because they experienced unfriendly services at CHC. As a note; it is more expensive to go to the clinics. The staff probably get paid better than CHC's staff and are therefore friendlier.

Previous researchers have found that waiting time affects people's perceptions about the given services and in turn, patients would choose to get a treatment from another health care provider the next time (Akhter et al., 2015; Alijanzadeh et al., 2016). However, the HCS in Jakarta, especially CHCs, face challenges to implement shorter waiting times. In Jakarta, there were too many CHC patients while there were too few general practitioners (Jakarta Provincial Health Agencies, 2016). The use of the national health insurance has resulted in each general practitioner to treat about 100 patients each day; consequently, each patient only had about four minutes for the overall treatment (Aziza, 2013). In addition, the duration for medical examination is limited due to the shorter service hours (i.e. 07.30AM-4.00PM; break 12.00PM-1.00PM) (Health Services of DKI Jakarta, 2015). The outcomes to the limited opening hour at CHCs with an overload of patients are longer waiting times and shorter examination times, as experienced by our participants. Consequently, our participants expressed a more negative view towards the CHCs' services and they chose private clinics and hospitals over CHCs. This preference might have also been influenced by the fact that most private clinics and hospitals provide 24 -hour services.

The use of modern and appropriate technology is one important aspect for the users of HCS (European Patients' Forum, 2017; Khamis \& Njau, 2016). Our participants admitted that they did not go to the $\mathrm{CHC}$ to get a medical treatment because they perceived the medicines to be of low quality, and the equipment to be old and inadequate. For them, it is useless to go to the $\mathrm{CHC}$ because for in-depth medical examinations, they would be referred to a hospital or a laboratory upon their CHC visit anyway. This view was in contrary to the statement of one lady who has a baby, she was confident with the quality of the vaccination provided by the $\mathrm{CHC}$, and doubted it when it came from private clinics. This belief might be related to the news about bootleg vaccines that came into view prior to the FGD. Even though the Indonesian Health Ministry published the list of convicted hospitals and clinics (Lembur, 2016), our participant might have held her doubt.

A low cost is one of the reasons why people use health facilities (European Patients' Forum, 2017). In Indonesia, the government promotes the use of the national health insurance since 2011, which makes medical treatment and medicines cheaper, or even free of charge (Ministry of Health Republic of Indonesia, 2014b). However, to get the benefit of the national insurance, one must visit the $\mathrm{CHC}$. If the CHC's general practitioner refers the patient to another HCS, the treatment and medicines will be covered fully or partially (The President of the Republic of Indonesia, 2011). This benefit leads to a positive view of the $\mathrm{CHC}$ by our participants; they prefer to go to the $\mathrm{CHC}$ rather than to private clinics or hospitals to get the benefit of the low cost. There 
were cases in which the participants went only to $\mathrm{CHC}$ to get the referral letter, to get a better treatment at a bigger hospital. Some patients even force the general practitioner to give them the referral letter because the CHCs' service is considered to be insufficient (Suprianto \& Mutiarin, 2017). In contrast, some participants directly go to the private clinics or hospitals even though it is expensive. They did not mind paying more because for them friendly and comprehensive services are the most important aspects.

In this study, the different HCSs mentioned by the participants had their advantages and weaknesses. In some cases, the participants did self-treatment because buying medicine at stalls is cheaper than going to a nearby clinic. If they did not get better, then they will visit either the $\mathrm{CHC}$ or the clinic. Self- and traditional treatments are used because it is a heredity tradition, and people believe in its effectiveness (Nurhayati \& Widowati, 2017; Peltzer \& Machleidt, 1992; Triratnawati, 2010). Most of our participants thought that in cases in which their sickness was a minor illness, they did not require a medical treatment from a general practitioner or a medicine subscription. On the other hand, self-treatments might cause the illness to get worse and the CHC's medicines might no longer have an adequate efficacy to treat this developed illness (Amegbor, 2017; D’Ambruoso et al., 2009). Traditional medicine is not prohibited by Indonesian government as stated in Act of the Republic of Indonesia number 36 of 2009 about Health (The President of the Republic of Indonesia, 2009). It emphasized on Regulation of Ministry of Health Republic of Indonesia number HK.01.07/MENKES/187/2017 (Ministry of Health Republic of Indonesia, 2017c) which underlined that citizens can use traditional medicine as long as they consult with a health care worker, use it rationally by following the instructions without neglecting the general practitioner's treatments or prescriptions. However, this information is unknown to most people, which might be due to the different understandings about self- and traditional treatments between patients and health care providers (Amegbor, 2017; Punamäki \& Aschan, 1994). The mismatches in understanding might result in discouraging patients to discuss the use of self- and traditional treatments with their general practitioners (Amegbor, 2017; Stevenson, Britten, Barry, Bradley, \& Barber, 2003).

The needs of psychological services appeared as an additional information. However, it confirms the findings from our previous study (Sokang et al., 2019a) which showed that a psychological need is of significant value for the community to be healthy. Psychological health is important, both in relation to physical aspects as well as in social aspects, and one's ability to carry out daily activities. In contrary, the presence of psychologists at CHCs in Jakarta is limited. Psychological services are only available in private practices, hospitals and clinics (Carina, 2017). Turning to Jogjakarta, one of the provinces of Indonesia, psychological practices have been provided in CHCs since 2010. CHC's psychologists detect early mental disorders in the community and they conduct prevention programs for mental health (Setiyani, 2018). Our previous study showed that participants needed psychological services but the service was not available at the $\mathrm{CHC}$. The participants expect the $\mathrm{CHC}$ to provide psychological services in order to help them deal with daily problems and severe mental disorders. 
The findings of this study provide insights in issues that are considered important by the community, such as staff's hospitality in HCS. The willingness of the participants to pay more in order to get friendly services shows the value of friendly services to the community. Friendly services make people comfortable to visit HCS. Beside the service improvements, the medical equipment quality used at HCS also one of the community's concerns. The use of modern and good-quality equipment would increase the community's trust in HCS. In addition to medical equipment, the quality of the medicines also needs to be improved so that people can get a more efficient medical treatment at the CHCs.

The government should pay more attention to the cultural aspects of health, such as taken into account the use of traditional medicines. Valid information with regard to how traditional medicines are regulated needs to be provided, both to health care providers and the public. Dissemination of the government regulations, such as regulation number HK.01.07/MENKES/187/2017 about the ingredients of traditional medicines in Indonesia is necessary so that people become aware of the proper instructions to take traditional medicine. By addressing the issue with traditional medicine, future patients might be more willing to openly discuss the use of traditional medicines with formal health workers and in turn, this interaction would help to increase the overall level of health in the community. The medical team should be aware that their negative reactions toward traditional treatments could influence how their patients share their alternative medicine consumptions and seek medical treatments in the HCSs. Another effort to consider is to involve traditional medicines practitioners in HCSs. This approach has been implemented in other countries with positive results (N. Robinson, 2006; Welcome to Country, 2018; Xu \& Yang, 2009).

There are strengths and limitations of our study that are worth mentioning. Firstly, to the best of our knowledge, the data on how the Jakartans value the current HCSs are rare, particularly the data taken with a qualitative approach. Secondly, the generalizability of the present results may be limited due to the small number of participants. At the same time, our data includes responses from participants from different ages and educational levels. On the other hand, this study may be subject to a potential bias due to the sampling method used. Using this research as an initial step, we suggest future studies to conduct multi-site studies with quantitative or mixed methods involving larger samples from other areas of Jakarta, with more diverse demographic backgrounds. The study could explore the other themes or issues that might be emerge from a broader sample, or focus on the perception or experience of specific age- or sex-group. Another study might explore the overall satisfaction of the community, as well as evaluating the treatment effectiveness.

\section{Conclusions}

This study explored the Jakartans' perceptions on HCSs, focusing on the hospitality of the staff members and the services of staff members and general practitioners. Overall, our participants expressed their preference to private clinics and hospitals 
over the government-mandated CHCs. CHCs had long waiting time and short opening hours, while private clinics had friendlier services, shorter waiting time, and longer opening hours, even though they had to pay more for the services. At the same time, the participants expressed a more positive view on the $\mathrm{CHCs}$ with regard to their service fees, which were affordable or even free under the national health insurance coverage. In summary, these results show that the current services offered by HCSs in Jakarta do not meet the needs of their communities. To alleviate this problem, we suggest the CHCs to take into account the needs and values of the community, including, but not limited to, incorporating a more open view on traditional medicine uses and providing psychological services. We hope that these recommendations would be taken into account in the future health-related policies and programs in Jakarta. 


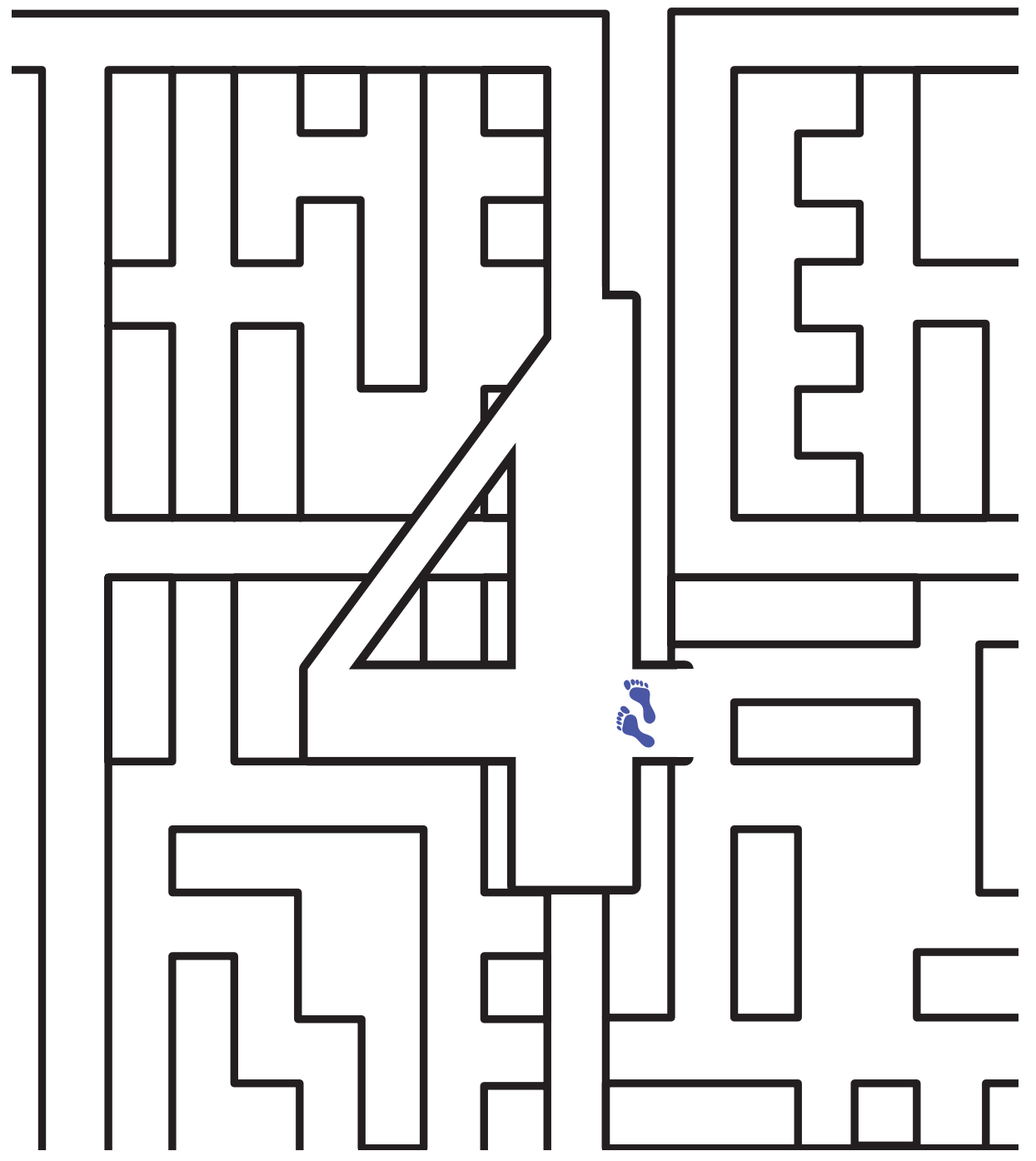




\section{Chapter 4.}

\section{General Practitioners' \\ Reflection on Health Promotion}

Based on:

Sokang, Y. A., Westmaas, A. H., \& Kok, G.. General practitioners' reflection on health promotion (Manuscript in preparation) 


\begin{abstract}
The Indonesian Community Health Centers (CHCs) use public seminars to disseminate health promotion. However, this approach has been heavily criticized. In this study, we conducted focused-group discussions with 46 general practitioner interns at CHCs to probe their perspectives with regard to the current health promotion programs in Jakarta. The results were analyzed using a thematic analysis which combined human coding and NVIVO-12. Overall, our participants had a negative view on the implementation of current health programs at CHCs; they were currently understaffed, which means that there was less time to educate the community members and increase their health awareness adequately. As a result, the community members preferred to apply selfand traditional treatments for health-related issues. To alleviate this problem, the participants suggested the Jakarta government and policy-makers enlist the help of community leaders, clinical psychologists, and religious leaders to help with health promotion programs at the CHCs.
\end{abstract}




\section{Introduction}

Community Health Centers (CHCs; Indonesian: Pusat Kesehatan Masyarakat, Puskesmas) are mandated by the Indonesian Government Regulations (No. 47/ 2016) (The President of the Republic of Indonesia, 2016) and are considerably important because they are on the front line in providing health care services to the community in every sub-district in Indonesia. One of the CHCs' mandatory health programs is health promotion, given that promotive and preventive efforts are the main concerns of the CHCs' programs (Ministry of Health Republic of Indonesia, 2014a). Health promotion in the $\mathrm{CHCs}$ is referred to as an effort to empower the community to prevent disease and improve the health of each individual, family, and environment independently, and to develop community-based health efforts (Ministry of Health Republic of Indonesia, 2007, 2011). The implementation of health promotion is regulated in the Minister of Health's decree number 585/Menkes/SK/V/2007 about guidelines for implementing health promotion in the CHCs (Ministry of Health Republic of Indonesia, 2007, 2011), with programs such as Posyandu (Indonesian: Pos Pelayanan Terpadu; English: integrated service post.), Posbindu (Indonesian: Pos Pembinaan Terpadu untuk Penyakit Tidak Menular; English: integrated coaching post for non-communicable diseases.), clean and healthy behavior program, immunization and vaccination programs, etc. (Directorate of Health Promotion and Community Empowerment, 2016).

To date, an evaluation regarding the effectiveness of health promotion programs, both in general and that are conducted by the CHC's, is not available in the Indonesian Ministry of Health performance reports (Ministry of Health Republic of Indonesia, $2017 \mathrm{a}, 2018 \mathrm{~b}$ ). The indicators of achievement only focus on specific issues, while the strategic goal is more general. For example, the first strategic goal is to improve the health status of the population. However, the performance indicators focus on pregnancy, and labor and delivery. The second strategic goal is to increase prevention and control of diseases, yet the performance indicators focus on specific diseases, such as treatment of tuberculosis, the prevalence of human immunodeficiency virus, malaria, leprosy, etc. (Ministry of Health Republic of Indonesia, 2017a, 2018b). In a similar way, a CHCs' performance can determine their accreditation. Yet, the performance indicators do not provide an evaluation of how $\mathrm{CHCs}$ conduct their health promotion activities (Ministry of Health Republic of Indonesia, 2015).

Following the specific performance indicators, the CHCs focus their health promotion more on raising public awareness of non-communicable diseases, including early screening and detection (Mahendradhata et al., 2017). The efforts to increase awareness are limited to (only) providing information to the community in health seminars (Indonesian: penyuluhan) during health promotion programs (Australia Indonesia Partnership for Health Systems Strengthening, 2016; Mahendradhata et al., 2017). With regards to early screening and detection, the CHCs focus more on curative rather than preventive approaches, which focuses on treating people who are already sick (Australia - Indonesia Partnership for Health Systems Strengthening, 2016). 
This might be related to the community's perception of the CHCs as a place to get treatment (Sokang, Westmaas, \& Kok, 2019c). At the same time, the lack of evaluation on the CHCs' performance makes it difficult for policy makers and health promoters to understand to what extent the CHCs' health promotion efforts have succeeded or failed.

To provide effective health promotion, it is very important to understand the behavior and environmental determinants of the community in order to properly address the health-related issue (Bartholomew Eldredge et al., 2016); in this case, the community is the $\mathrm{CHC}$ itself. One approach that emphasizes the importance of community assessment is Intervention Mapping, which contains six steps to follow, with several tasks at each step to complete (Bartholomew Eldredge et al., 2016). This approach emphasizes the importance of theory and evidence as the basis of building an intervention, which includes planning and designing, developing, implementing, and evaluating the program objectives (Bartholomew Eldredge et al., 2016).

In order to recognize the determinants of the CHC as a community, we used the first step of IM, the needs assessment, to understand the delivery of health promotion by CHCs in Jakarta. It should be noted that the present study took the CHCs general practitioner's (GP) perspective to explore the challenges and obstacles on conducting health promotion in Jakarta's community. In the CHCs, the GP has a significant role. Their tasks are not only to treat the patients, but also to do advocacy for patients in terms of health. GPs are expected to provide education, information, and support while approaching, understanding, and empowering the patient, the patient's family, and the community. Therefore, GPs comprehend the patient's health journey (Megawangi, 2017; Widiyani, 2014). We considered the GP's perspectives as a necessity to achieve a better understanding of their experience. This knowledge will guide us to a more tailored way to improve the health promotion in the community (Jormfeldt et al., 2007; Mendelson, 2002).

\section{Methods}

\section{Data Collection}

To capture a comprehensive perspective of GPs on health promotion activities carried out by CHCs in Jakarta, focus group discussions (FGDs) were conducted by the first author and a team of four-trained research assistants (graduate bachelor students in psychology). The assistants were trained to do the observations and to make notes during FGDs, and to conduct an analysis of the data together with the first author. Due to unclear procedures and slow-response in getting permission from the Health Office of West Jakarta Municipality, the available time for data collection was unexpectedly limited. Therefore, the first author decided to recruit GP interns -as opposed to GPswho were in their internship at several CHCs in Jakarta.

After obtaining permission from the Faculty of Medicine of Krida Wacana Christian in Jakarta in October 2017, the first author attended two classes of mentoring activities 
in order to personally recruit participants. The purpose of the study was explained while the first author provided an opportunity to ask questions regarding the study. The potential participants were invited to leave their contact information after which the research assistants contacted with an official invitation.

Four FGDs (min. 79 - max. 106 minutes) were conducted at the Faculty of Medicine of Krida Wacana Christian University. A total of 46 GP interns were voluntarily involved in the group discussions, using the Indonesian language. The first author led the FGDs supported by the team. The FGDs were recorded (video and voice recorder) after participant's approval by signing the informed consent forms. The guideline questions were, i.e. 'What are your activities during the internship in CHCs?'; 'What are the health promotion activities?'; 'What do you think about it?'. Unclear statements were clarified by probing questions, such as 'Can you give an example?'; 'Can you explain?'; 'Why?'.

This study was approved by both the Ethics Review Committee of the School of Psychology and Neuroscience, Maastricht University (Reference number: ERCPN 0409-2012_S21), and by the Grogol Petamburan sub-district official (letter number 424/ UKKW/FPsi/Prodi/X/2017). The approval of the GP interns' coordinator was acquired orally, as the first author is an employee of the same university. As stated above, the researchers obtained a written agreement to participate from participants. Due to confidential consent to the participants, data are available upon request by contacting the corresponding author.

\section{Analysis}

We followed an inductive content analysis approach using a combination of human coding and NVivo-12 coding. In the human coding process, firstly, the team transcribed the voice recordings, and the transcriptions were reviewed by the first author to ensure the transcriptions were correct. Secondly, the transcripts were analyzed with the process of open coding and creating categories (Elo \& Kyngäs, 2008; Hsieh \& Shannon, 2005). The research assistants individually performed the process of open coding. To create categories, the first author and the team discussed the coding result until consensus was reached (Leung, 2015; Noble \& Smith, 2015). Inductive thematic saturation was applied; saturation of the data was achieved when no new category or theme emerged from the texts (Saunders et al., 2018).

In regards to the Nvivo-12 coding process, firstly, the transcriptions were translated into English by the first author and reviewed by the second author. Next, the first author performed open coding and creating categories process in NVivo-12. The result of human coding and Nvivo-12 coding were then compared to ensure that both analyses would fit each other. There was no major difference found between the coding. 


\section{Results}

In total, 30 female and 16 male GP interns participated in four FGDs in the age of 2225 years old. We did not differentiate the participants' perceptions based on sex, age, or other demographic data, as that was not our purpose. Therefore, the shared perceptions will be reported, unless stated otherwise. In the result and discussion section, we separate the statement of our participants into two main sections: reflections on $\mathrm{CHCs}$ as the health promoter; and reflections on the community as a target of the health promotion. Further, we present the recommendations that were proposed by our participants.

\section{The CHCs as health promoters}

Our participants were dissatisfied with the health education provided by $\mathrm{CHCs}$ both in and outside of the CHC. Due to the limited number of staff, GP interns were often assigned to go into the community for public seminars or monthly programs, such as on giving immunizations, as stated by one 23-year-old male participant: “... for the seminars, usually done by the nurse or the receptionist, or the interns."

Another concern regarding the CHCs' role as a health promoter was the limited time that the GP had to educate patients. Compared to the numbers of visitors, the number of CHCs' staff was insufficient. The GP cannot provide proper education to the patients because "the time to educate the patient is too short", as one 23-year-old female puts it. In addition, GPs cannot refer patients to a more suitable professional, such as a nutritionist or midwives, because those were giving services outside the CHC. One 23-year-old female stated: "Maybe due to lack of staff, because the nutritionist must also be at Posyandu, and Posbindu. She's only one person, and she has to stay at the CHCs, also, she must do the program in the community too". When asked about the proper number of health workers for the $\mathrm{CHC}$, participants agreed that the number of $\mathrm{CHCs}$ ' staff is actually enough if they were only working inside the CHC. However, they need a different team to go out to the community: “... seven people are enough at the CHC. But maybe should add one or two more doctors, or two nurses to do another program outside the CHC. So, the CHCs'service can be done, and other programs can be done as well."

In regards to the $\mathrm{CHCs}$ ' role in giving public seminars of health, there were two concerns expressed by the participants. Firstly, the theme of the public seminar was too broad, going into various aspects of a disease: "So, not all information is absorbed by the listeners." Secondly, the wide topic of the seminar is the result of targeting unsuitable groups in the community. As an example, another 23-year-old female reported "For the content [of the seminar]. For people who are not exposed yet, the content is about exposure prevention and the complications. For people who are exposed, the content is more about the treatment. For example, for disease that require to drink the medicine daily. We give seminar about the medications. So, we get them interested, like give them motivation so that they know the medicines should be taken daily. We don't give education about what is the disease, or things like that. So, they understand more. They 
are more curious, because it related to them. If they are not exposed yet, they will avoid it because we already gave seminar how to prevent it. So, it's the content."

\section{The community as a target of the health promotion}

Participants also expressed reflections on the community as the target of health promotion as it is believed that they lacked awareness on practicing healthy living. Although the participants acknowledged that it was most likely related to the lack of education provided by the $\mathrm{CHC}$, participants also believed that the community was rather careless about their own health. It was illustrated by a statement from a 23-yearold female participant “... There's no willingness from the person... Even though they eventually suffer from high blood [pressure], cholesterol... They put it, like, ah today [I can] eat a lot of cholesterol [food], it's ok, there's medicine. Or, for example, smoking, ah, [I'll] just keep smoking, then [I can] take vitamins to be healthy." The community underestimates the severity of the disease because there will be doctors to treat them. As one 23-year-old female stated "because they think, ooh, my blood sugar is high, well, I can just go to the doctor, and get some medicines." On the other hand, there were people who refused modern medicine and insisted on getting traditional treatments. Another 23-year-old female reported "they believe that if they take medicines continuously, it will damage their kidneys. Whereas for example, drinking jamu (a traditional herbal medicine from Indonesia), it is herbal, natural for the body, so it's healthier".

Regarding the self- and traditional treatments, our participants had a negative attitude because they believed that Indonesian traditional treatment practices were not based on research. Moreover, they often treated patients in a more advanced stage of a disease because the patient got a traditional treatment before. One 23-year-old female stated that "most people come to the hospital or to the CHCs in a severe condition, and before that [, they were] from alternative medicine. The person's disease actually got worse because of the alternative [treatment]. When it's getting bad, nothing can be done, just then they take the person to a doctor".

\section{Aspirations}

While reflecting the CHCs' health promotion programs, our participants expressed their aspirations and what they expected from the Indonesian government and Ministry of Health. They proposed that every CHC should have a field team; a special team with the tasks of go out to the community to promote health, educate the community, and also to do monthly programs such as immunizations, vaccinations, Posyandu, and Posbind $u$ programs. With the field team involved, our participants assumed that the CHCs' staff can focus more on services inside the $\mathrm{CHC}$, while the field team focuses on establishing relationships with the community. The field team can collaborate with health cadres of the village, as the health cadres are members of the community who voluntarily help the CHCs' health promotion programs and trained by the CHC. One 22-year-old female stated "The community might be a bit reluctant to tell us a story. So, the cadre is chosen so that there is a liaison between the community, cadres, and directly to the CHC's 
staff... They have been trained, indeed." However, some participants argued that not all cadres were actively involved in the programs or have proper skills.

The participants also expressed the need for clinical psychologists in the CHC. According to them, it would be beneficial if the clinical psychologist has a schedule to go to different CHCs per day. Therefore, the GP refers patient only on the scheduled day, as expressed by one 25-year-old female "There is a need for a clinical psychologist to go to CHCs per day. There's a schedule. So, not every day [the clinical psychologist stays] at one CHC'. The other option is incorporating the clinical psychologist to the field team in order to early-diagnose the mental-health patient in the community.

Our participants also proposed an idea of collaborating with religious leaders in promoting health. Our participants believed that the involvement of religious leaders might increase the success of government programs, such as immunization program. One 22-year-old female argued that "In my opinion, a religious approach is indeed important because Indonesian, they firmly believe in religion; moreover, we are the largest Muslim country. It is important, I think, because whenever we use religion, they will be more interested, more aware about it. Sometimes the people trust the priest more than the doctors".

During the discussion, the participants compared the services at their CHCs. Some participants claimed that psychological services had been carried out in their CHCs, while the other stated that their CHCs had no such program. The participants indicated that this difference in practice was because every $\mathrm{CHC}$ had the autonomy to manage funds they got from the government, which contributes to the differences in health efforts carried out by the CHCs in each region. One 22-year-old female reported "[The funding is] different. First, it depends on the community scope. Second, it depends on what the diseases are, the frequency, the diversity, and the severity [of the diseases]. For example, in coastal areas, there are many sexually transmitted diseases. Then the CHCs will spend money specifically for the STD prevention program. For other regions, [they] do not necessarily do that".

\section{Discussion}

The study aimed to explore the challenges and obstacles on conducting health promotion in Jakarta from the CHCs medical staff's perspective. Two main concerns were expressed by the participants regarding health promotion in Jakarta. Firstly, concerns about CHCs' as the health promoter, including the burden of giving services inside and outside of the CHC; the limited number of staff; the limited time to educate patients; the broadness of the topic of the public seminar; and the unsuitable target groups for the seminar. Secondly, concerns about the community as the target of health promotion programs, including lack of awareness on healthy living; and the tendency of using selfand traditional treatments. 
There are a few points to keep in mind while interpreting the results. The participants of this study were GP interns who were in their internship at several CHCs in Jakarta. The authors originally intended to recruit CHCs staff as participants. However, due to time constraints and difficulties in getting a research permit from the West Jakarta's Health Office, we decided to recruit the interns instead. As a result, the findings might not be transferable to all the CHCs in Indonesia because our participants were interns as opposed to full-time staff. Despite these considerations, the authors believe that the results are valuable. Evaluation reports about the $\mathrm{CHCs}$ ' performance in health promotion are rarely available, especially evaluations by the medical staff themselves. This study provides a good start to promote self-evaluation among health providers. The participation of the interns in the FGDs provided renewed perspectives on health promotion. They are not employed by the CHCs and therefore, can be considered to be less biased towards CHCs. However, at the same time, they can be biased due to their vulnerable position as an intern. The participants did their internship at several CHCs in Jakarta and for that reason they were capable to compare the health promotion programs between CHCs in Jakarta. They received training in public health and health promotion as part of their education, and are thus able to compare their trainings with the application in real life. For these reasons, regardless of the shortcomings, we consider the characteristics of our participants as an advantage.

Efforts on health promotion programs have been carried out by the Indonesian government, for example the Ministry of Health sets four targets to achieve until 2019, namely a) to improve the health and nutritional status of the community; b) to improve control of communicable and non-communicable diseases; c) to improve equity and quality of health services, and d) to improve financial protection, availability, distribution, quality of medicines and health resources (Ministry of Health Republic of Indonesia, 2018a). Most of the targets have become the CHCs' performance targets since they are in the front line of health services in Indonesia. On the other hand, the number of medical staff at $\mathrm{CHCs}$ is inadequate, with one doctor, one nurse and one midwife, who together are responsible for 5,000 people (Ministry of Health Republic of Indonesia, 2017b; Putri, 2013; WHO Country Office Indonesia, 2016). Another consequence of the lack of staff is the negative perspectives of the community towards health services in Jakarta as shown in previous research (Sokang et al., 2019c). One of the reasons is the long waiting hours to get examine because the doctor has a program to do outside the $\mathrm{CHC}$.

The Indonesian government is aware of the staff's shortage, which resulted in the involvement of health cadres in health promotion programs. However, there are cadres who have insufficient skills to do the tasks (Caturiningsih, Mestuti, \& Atik, 2016; Qusna, 2016; Tristantia \& Khoirunnisa, 2018). Already in 1984, Berman has stated that the village-level health worker -or nowadays called health cadres- programs rapidly expanse, therefore it requires continued research to evaluate the coverage, the equity, the health outcomes, the costs, and the participation (Berman, 1984). Despite that, recent studies show that the training given to the cadres is brief and not practice-oriented, resulting in cadres that lack the knowledge and skills to communicate effectively with 
the members of the community (Nazri et al., 2016). Even though stated as the backbone of the Posyandu, no evaluation of the health cadres programs can be found in the review of the health system in Indonesia (Mahendradhata et al., 2017).

In response, our participants suggested that the government should form a special field team, which focuses on health promotion in the community, including doctor, nurse, midwife, and clinical psychologist. Thus, the target from the Health Ministry can be achieved without sacrificing the service at the CHC. Working as a team in the community is not a new topic in health promotion and community-based research. One of the popular strategies is community coalition (Butterfoss, Goodman, \& Wandersman, 1993; Butterfoss \& Kegler, 2012). The strategy focuses on reducing or preventing issues in the community, and is mostly used to promote public health (Arthur et al., 2010; Yang, Foster-Fishman, Collins, \& Ahn, 2012). The community coalition emphasizes the involvement of diverse members from both grassroots and professional levels (Butterfoss et al., 1993). Hence, the members have direct experience with health issues, besides the social and cultural context of the issues. Therefore, the coalition is able to make decisions that are in line with the values and cultures of the community (Butterfoss et al., 1993; Butterfoss \& Kegler, 2012).

The Indonesian government and the health providers should also consider involving more parties in the implementation of health promotion programs, for example religious leaders. Multiple studies indicated the value of this collaboration (K. Hays \& Aranda, 2016; Schwingel \& Gálvez, 2016; Shirazi, Shirazi, \& Bloom, 2015; Yanek, Becker, Moy, Gittelsohn, \& Koffman, 2001). A study in Malaysia found that faith and religious acts influence people's health-related actions, directly and indirectly (Hassan, 2015). Consistent with this finding, a previous study showed that people's behavior towards health and sickness might be influenced by their perspective on health and sickness (Sokang et al., 2019a). Religious leaders can open discussion about the link between physical and spiritual aspects of health to community (Rivera-Hernandez, 2015; Sokang et al., 2019a), apart from being a role model (Baruth, Bopp, Webb, \& Peterson, 2015).

Discussing the health promotion programs cannot be separated from discussing the funding to do the programs. Our participants briefly mentioned the possibility of the influence of funding in the different activities at the CHCs. Funding from the Indonesian government varies depending on the CHCs' type and the number of the patients. Despite the government's recommendations and technical instructions on using the funding, the authority to regulate it is within the CHC itself (Ministry of Health Republic of Indonesia, 2019; Tarigan, 2015). However, with the funding, the CHCs focus their performance on achieving the targets from the Ministry of Health (Ministry of Health Republic of Indonesia, 2017a, 2018b); while overlooking the values and needs of the community. Therefore, it is important to raise the CHCs' awareness to use the funding for health promotion programs which are in line with the community's needs and values. 


\section{Conclusions}

Concerns regarding health promotion in Jakarta point to the CHCs as the providers and the community as the recipients of the program. The lack of staff makes it difficult for the CHCs to perform its main task in promoting health. They cannot provide the best services both inside and outside the CHCs. The involvement of community members, such as health cadres and religious leaders might beneficial for the CHCs in performing the task. On the other hand, the CHCs need to provide appropriate training, supervision and support to the community members so that their presence can be valuable. One strategy to connect the $\mathrm{CHCs}$ and the community is the community coalition program. This strategy can be used to counter the imbalance between the numbers of CHCs staff and patients. Coalition members include professionals, such as doctors, nurses, midwives, and clinical psychologists; and community members, such as health cadres, and religious leaders. Consequently, the $\mathrm{CHCs}$ funding can be used for health promotion programs that fulfill the needs and the values of the community, as the community is actively involved in the program itself. 


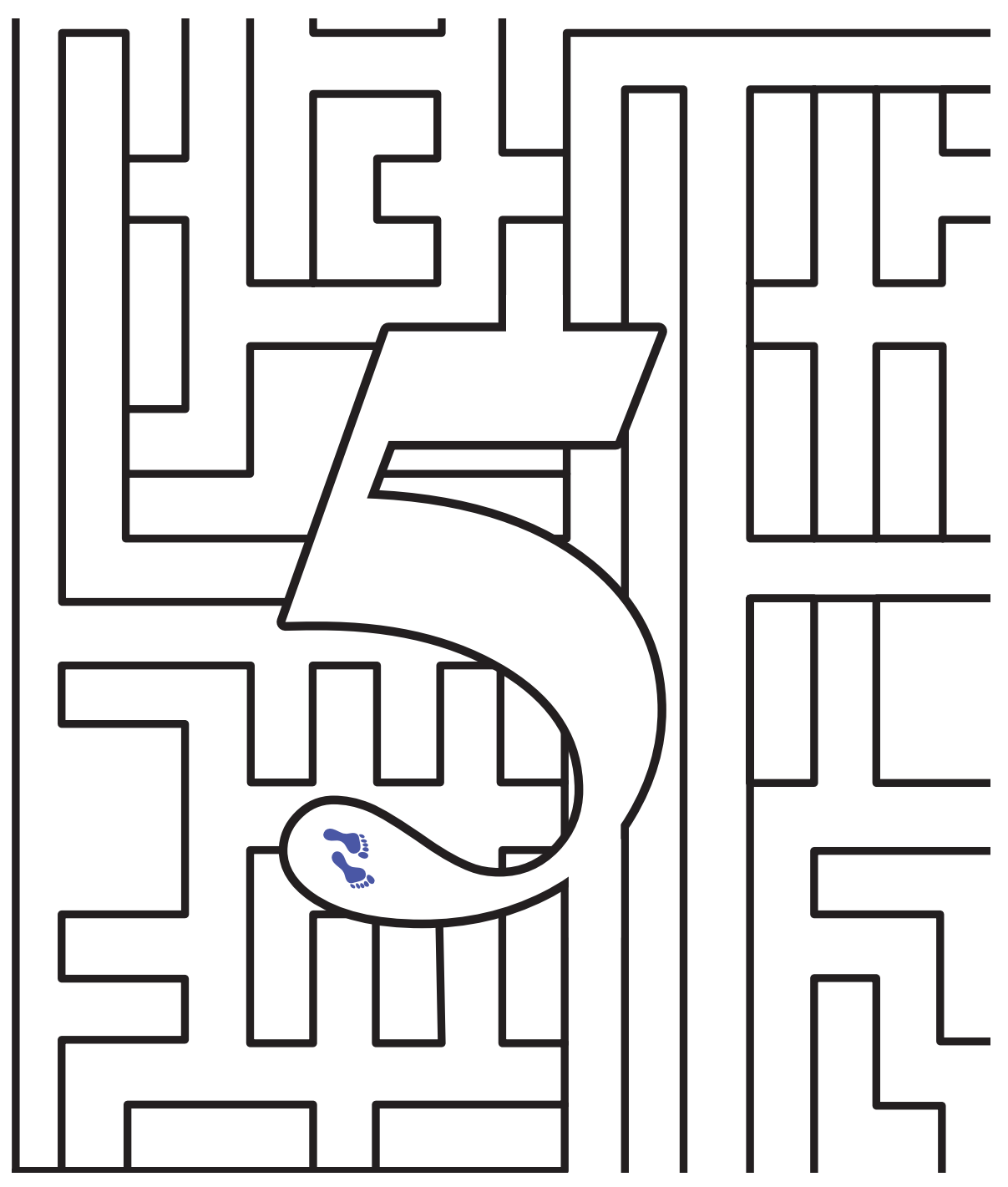




\section{Chapter 5.}

\section{Considerations in the Community Coalition Implementation: A Review of the Literature}

Based on:

Sokang, Y. A., Westmaas, A. H., \& Kok, G.. Considerations in the Community Coalition Implementation: A Review of the Literature (Manuscript in preparation) 


\begin{abstract}
This study organizes existing studies on the Community Coalition approach to synthesize aspects considered important in applying the approach for health promotion intervention in urban areas. From 816 articles found using PubMed, PsycINFO, and the snowballing approach, 10 records were extracted in the final literature review. Seven, partly overlapping, aspects were found: the diverse expertise of the members, the clarity of the goal, the member's interest and motivation, the leadership, the trust and cohesion of the coalition members, the clarity of the members' roles, and understanding the norm and the culture of the target community. The discussion addresses the importance of the findings to improve our understanding of the relevant aspects in applying the Community Coalition approach for health promotion intervention in urban areas.
\end{abstract}




\section{Introduction}

Health promotion is a major concern for health practitioners nowadays because it is inseparable from the effort to improve public health (Crosby, Kegler, \& DiClemente, 2002). Furthermore, more and more people are aware that improving health in the community must begin with preventive efforts, and health promotion program is one of the ways out (Fertman et al., 2017). Despite the considerable resources that have been dedicated to preventing prevention over the past decade, various difficulties are still encountered such as ineffective and unsustainable health promotion programs (Shortell et al., 2002; Soltani et al., 2017). One of the reasons is that the majority of the efforts the health promoters mostly work alone and focus on a certain aspect of health (Wong, 2015). While research has shown that health promotion demands a bottomup collaboration of professionals and grassroots to facilitate an effective and sustain health promotion intervention, and at the same time fulfill the needs of the community (Butterfoss \& Kegler, 2012; Laverack, 2007, 2014; McNeish, Rigg, Tran, \& Hodges, 2019; Shortell et al., 2002).

A popular approach to fill the gap between existing programs and the unfulfilled needs of the community in intervention development is the Community Coalition (CC). It is defined as a group of individuals representing diverse organizations, factions, or constituencies within the community who agree to work together to achieve a common goal (Feighery \& Rogers, 1990). CC emphasizes the partnership to jointly define issues that arise in the community, assess the determinants of health problems, identify, analyze, and arrange solutions, implement, and evaluate the issue in order to minimize or prevent a problem in the community (Butterfoss et al., 1993; Butterfoss \& Kegler, 2002). Even though the size of the coalition may vary, a coalition requires involvement of both professional and grassroots organizations (Butterfoss \& Kegler, 2012). Different from other approaches, which use an informal approach, CC is a formal -businesslike arrangement- approach with commitment for a longstanding collaboration (Butterfoss \& Kegler, 2012; Feighery \& Rogers, 1990).

To achieve a sustained and effective program, research had been conducted to find factors that build an effective coalition i.e. group size, member diversity, roles, norms, cohesion, culture, conflict resolution, decision making processes, and leadership (Levine $\&$ Moreland, 1998). Others found six coalition-building factors which associated with indicators of effectiveness: formalization of rules/procedures, leadership style, member participation, membership diversity, agency collaboration, and group cohesion (Zakocs \& Edwards, 2006). Other research found that the key metrics of the collaborative approach are coalition leadership, goals, member characteristics, engagement level, planning activities, decision- making processes, relationship, and availability/use of resources (Butterfoss, 2006; Butterfoss \& Kegler, 2002, 2009; Mizrahi \& Rosenthal, 2001). While the aspects mentioned are the most important, it could be a challenge to maintain a sustained coalition because it involves people from diverse organizations and backgrounds (Butterfoss \& Kegler, 2012). Many interventions found difficulties to enlist 
and sustain the collaboration (McNeish et al., 2019). The reasons are such as unclear goals and expectations of a partnership, and unclear or intermittent communication (Corbin, Fernandez, \& Mullen, 2015; Flores, Montgomery, \& Lee, 2005; Soltani et al., 2017).

The present study is a follow-up of our previous studies (Sokang et al., 2019c; Sokang, Westmaas, \& Kok, 2019b; Sokang et al., 2019a), which found differences between the approach of health providers in promoting health and the values that are considered important by the people of Jakarta, Indonesia (Sokang et al., 2019c, 2019a). As a result, the community members preferred self- and traditional treatments, and only came to health services when the disease becomes more severe (Sokang et al., 2019b, $2019 \mathrm{c}$ ). Therefore, the health providers proposed to involve community members, such as health cadres/ volunteers, key leaders, and religious leaders to promote health in the community (Sokang et al., 2019b). As discussed in the previous study, the community coalition appears to be an appropriate approach to respond to the suggestions.

In contrast to the amount of research that has been done, to date only limited research has been done on the aspects that need to be considered if CC is carried out in urban areas, whereas more than half of the world population lives in urban areas $(68 \%$ as projected in 2050) (Krefis, Augustin, Schlünzen, Oßenbrügge, \& Augustin, 2018; United Nations - Department of Economic and Social Affairs - Population Division, 2019). Challenges to urban populations' health is complicated as the city provides both the source of healthcare and threats to the health (Galea, Freudenberg, \& Vlahov, 2005). There is also the contrast between characteristics of urban population and those who live in rural areas (Rydin et al., 2012). Given the complexity of health issues, maintaining the coalition, and the urban characteristics, the present study used a literature review to synthesize which aspects are considered important in applying the $\mathrm{CC}$ approach for health promotion intervention in urban areas.

\section{Method}

The search strategy used in the present study consisted of three steps. First, the databases PsycINFO and PubMed were searched using the key words 'Community coalition', 'Community-based coalition', 'Community coalition-driven', 'Urban', 'Intervention', and 'Health promotion'. The first and second author read the articles to find the commonly used term to describe the community coalition. From initial searching, several synonyms to CC were found, such as 'Community-based coalitions', 'Community coalition-driven, 'Community alliances, 'Collaborative partnership', 'Health Care Coalitions', and 'Intersectoral partnership'. The second round included the new keywords in searching. Lastly, we used the snowball method to draw other articles. The following criteria for inclusion and search strategy were used to identify research articles that could answer the question. 


\section{Search Strategy}

We used two electronic reference databases to search the literature: PubMed, and PsycINFO. Using the keywords mentioned, the search was limited to publications from 2010 to 2019 in English language peer-reviewed journal. The first and second author independently reviewed the articles to determine whether the articles did meet the inclusion criteria or not. The final articles were synthesized using qualitative approach by creating themes.

\section{Inclusion/exclusion criteria}

Publications were only included in the analysis if they met the following criteria:

1. Health promotion intervention program/ study worldwide targeted urban neighborhood/ urban area.

2. An evaluation of health promotion intervention program/ study which using community-coalition approach.

3. Demonstrate that the community-coalition approach is used to improve health status for both, general or variety health issues and specific health issue.

4. Define and measure the effectiveness of the community-coalition approach, either quantitatively or qualitatively, and describe the indicators of the effectiveness.

5. Define and measure the coalition-building factors (group size, member diversity, roles, norms, cohesion, culture, conflict resolution, decision making, and leadership.) and assess how or whether the coalition-building factors are related to the indicators of coalition effectiveness.

6. Published in a peer-reviewed journal from 2010 to 2019 (the last day of searching: 14 November 2019).

\section{Results}

816 articles were found, with 60 articles from PubMed and 756 articles from PsycINFO. Another 62 publications were found through the snowball approach. The results from these searches were scanned manually for duplicates. The 871 publication titles and abstracts that remained were assessed for relevance. However, 367 articles were discarded because they did not meet the inclusion criteria, such as not being research articles or were not health-related. Another 22 articles were discarded due to lack of availability full-text article. From the remaining 482 articles, 473 publications were excluded because they did not address community intervention, community coalition study, or health-related issues sufficiently, nor assessed and described the CC's indicators of effectiveness. As a result, 9 records were included in the final literature review (figure 2). The overview of included studies can be seen in table 4 .

The results show that the studies used different models in measuring the effectiveness of the coalitions. They also used different description of the results. However, the results of evaluations conducted by these studies emphasized the key findings that can 
be merged into seven recurring themes, such as the diversity of the coalition member, the clarity of the coalition's goal, member's interest and motivation, the leadership, the trust and cohesion of the coalition member, the clarity of the member's roles, and understanding the norm and the culture of the target community.

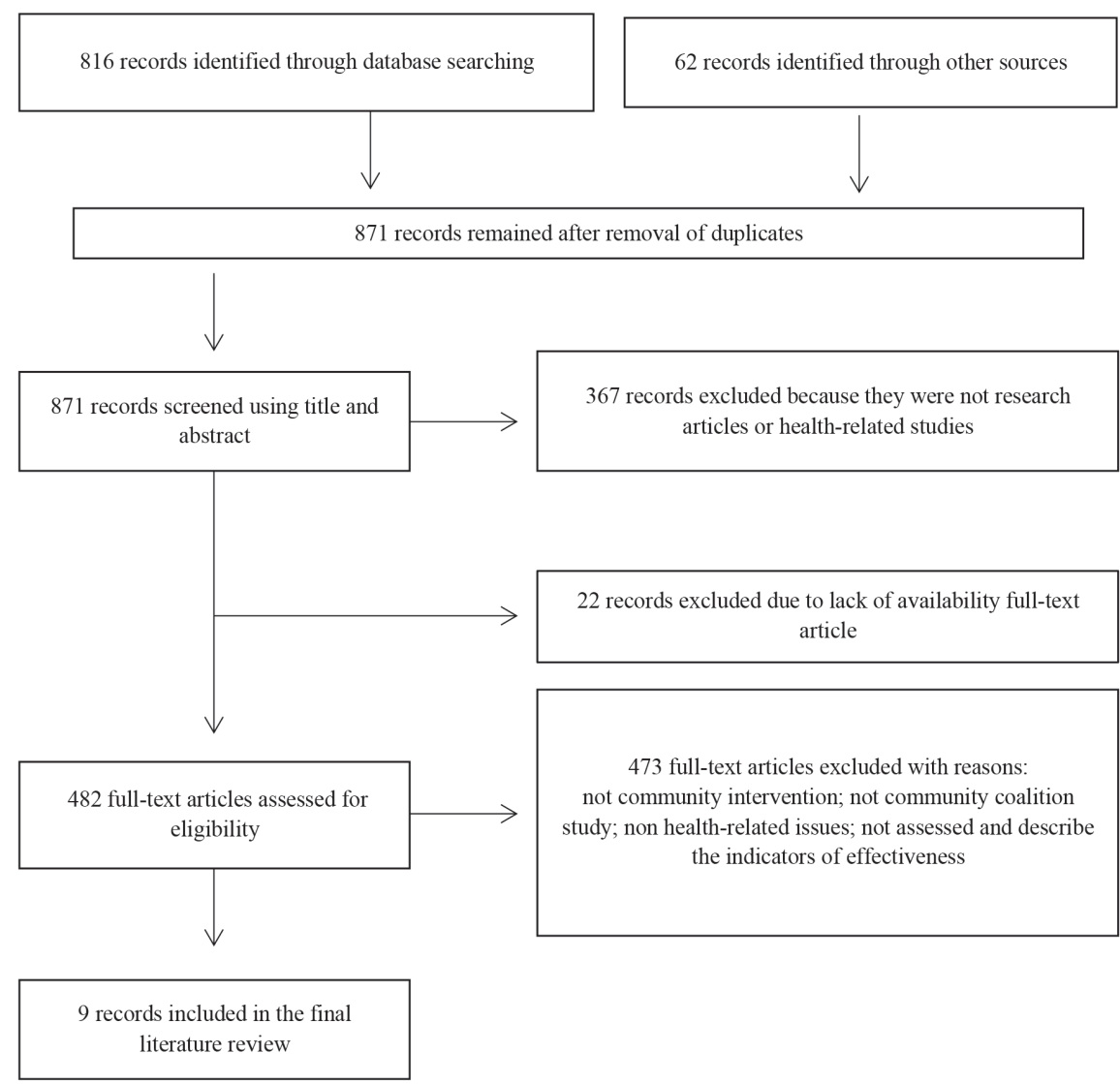

Figure 3: Flow diagram with results of each step in the literature search

\section{Member diversity}

The literatures found that member diversity is crucial in building a coalition. A diverse coalition members from cross section interested professionals and different fields benefit the coalition because they have different expertise, knowledge, skills, and resources (Anderson-Carpenter, Watson-Thompson, Jones, \& Chaney, 2017; Clarke-McMullen, 2010; Doll et al., 2012; McNeish et al., 2019; Motley et al., 2013; Peterson et al., 2019; Reed, Miller, \& Francisco, 2014; Reid et al., 2018). With diverse members, the coalition has access to a variety of resources, both physical and non-physical resources, which the professionals have. These resources is useful to improve the member's abilities, not only 
sharing skills and knowledge to develop and implement programs, but also share facilities, materials, and networking (Anderson-Carpenter et al., 2017; Doll et al., 2012; Motley et al., 2013). As a result, the availability of these resources helps the coalition pursue and achieve its goals (Anderson-Carpenter et al., 2017; Doll et al., 2012). Therefore, other than the professionals, it is crucial to connect and build relationships with the stakeholder in the community, i.e. key community leaders and policy makers (Reed et al., 2014; Reid et al., 2018); since the community members are the key contributors due to their insight and understanding of the issues in the community (Clarke-McMullen, 2010). The diverse partnership members will also benefit the coalition in a long run. The members work effectively because a problem can be solved together, and reduce the chance of burn-out of a "dedicated few" coalition members (Doll et al., 2012; Peterson et al., 2019; Reid et al., 2018). Working with diverse resources from the members will also benefit financially as the coalition is able to use the funding efficiently (Motley et al., 2013). At the same time, the members will willingly share their resources when they trust the coalition, and are confident that the coalition heads toward the same goal and interest as theirs (Anderson-Carpenter et al., 2017; Clarke-McMullen, 2010; Doll et al., 2012; McNeish et al., 2019; Motley et al., 2013).

\section{Ensuring goals and purpose clarity}

A clear purpose of a coalition is something that must be decided from the start (Anderson-Carpenter et al., 2017; Clarke-McMullen, 2010; Doll et al., 2012; McNeish et al., 2019; Motley et al., 2013; Reed et al., 2014; Reid et al., 2018), when the coalition understands the problem in the community (Motley et al., 2013). The understanding the problem is useful for the coalition to set goals (Motley et al., 2013; Reed et al., 2014; Reid et al., 2018). The coalition's ability to understand the issues, includes the ability to identify, solve, and act on the problem (Motley et al., 2013). Furthermore, the clarity of the specific goals will help the coalition members to develop effective assessments, clear strategies and action plans (Doll et al., 2012; McNeish et al., 2019), as well as to keep the coalition on track (Doll et al., 2012; McNeish et al., 2019; Motley et al., 2013; Reid et al., 2018). The shared purpose creates understanding within the partnership and allows the members to work as a team toward the common goals without 'wasting time and energy on internal conflicts' (Clarke-McMullen, 2010). The understanding leads to trust and appreciation as the members believe the coalition go toward the same direction as theirs (Doll et al., 2012; McNeish et al., 2019; Motley et al., 2013); compared to members who do not understand or agree with the goal and purpose may reluctant to engage in and to complete the coalition tasks (Doll et al., 2012). Moreover, a clear purpose enables the coalition to recognize skills, knowledge, and technical supports needed to achieve the goal (Anderson-Carpenter et al., 2017). The members are able to identify 'the right partners at the right time' (Reid et al., 2018). At the end, a clear purpose provides a clear end-goal which provides a clear indicator for evaluation (Clarke-McMullen, 2010). 


\section{Member interest and motivation}

In a diverse member group, ensuring the interest and motivation of the members is important to pay attention to; to make sure that the members are aware of the targeted issue and wants to achieve the same goals (Anderson-Carpenter et al., 2017; Clarke-McMullen, 2010; Doll et al., 2012; Motley et al., 2013; Peterson et al., 2019; Reed et al., 2014; Reid et al., 2018). Differences in interest can also indicate power differences; hence, members need to balance the power by an active and equal involvement in the coalition (Peterson et al., 2019; Reed et al., 2014). The involvement, in this case, is not only being active in the programs, but also develop a shared vision and mission (Clarke-McMullen, 2010; Doll et al., 2012; Reid et al., 2018); as well as engage in the identification of problems, create programs, and evaluate the programs (Clarke-McMullen, 2010; Doll et al., 2012; Motley et al., 2013; Reid et al., 2018). An equal and active partnership might increase the sense of community so that the members will continuously solve their own issues and sustain the changes (Motley et al., 2013). Additionally, the active involvement of all coalition members combined with the exchange of resources benefits the coalition to enhance members' confidence in addressing the targeted issue (Motley et al., 2013). Synergy in coalition's task is beneficial to the partnership in implementing the strategy successfully even without funding (Clarke-McMullen, 2010). Furthermore, it is particularly important to increase the knowledge and awareness of the members before implementing the programs of intervention, to make sure that all the members are well-educated and well-trained about the targeted issue (Anderson-Carpenter et al., 2017; Clarke-McMullen, 2010). Using the knowledge, the members can provide recommendations that are appropriate for achieving the goals of the coalition (Doll et al., 2012).

\section{Leadership}

Leadership, including governance structures and decision-making processes, is another important aspect to be considered in a coalition (Anderson-Carpenter et al., 2017; Brown, Feinberg, \& Greenberg, 2010; Clarke-McMullen, 2010; Doll et al., 2012; McNeish et al., 2019; Motley et al., 2013; Peterson et al., 2019; Reid et al., 2018). A leadership in a coalition expected to be a shared governance that provides places for multiple perspectives with consensus-based decision-making (Brown et al., 2010; McNeish et al., 2019). Similarly, the leader is required to address disparate visions among the members, understand their perspectives, unique priorities and interests (Doll et al., 2012) by maintaining an open \& frequent communication between members, sharing coalition resources, and keeping everyone up to date (Anderson-Carpenter et al., 2017; ClarkeMcMullen, 2010). The communication will benefit to reduce conflict that potentially arise in a coalition (Anderson-Carpenter et al., 2017; Clarke-McMullen, 2010; Doll et al., 2012). Apart from that, a leader needs to provide a supportive environment so that coalition members learn to understand their partners and practice how each member can serve the other (Doll et al., 2012; Reid et al., 2018). In making a decision, a leader expected to involve the member in order to make them feel they contribute achieving the coalition's goal (Clarke-McMullen, 2010; Motley et al., 2013), including during the 
preparation of rules, roles, processes, and coalition structures (Motley et al., 2013; Reid et al., 2018). Furthermore, a leader also needs to give attention to upgrade the members' skills and knowledge by training and sharing the latest issues related to the targeted topic (Clarke-McMullen, 2010; Peterson et al., 2019). Similarly, the leader needs to improve their own abilities, knowledge, and skills to keep align with the development and progress of the coalition (Motley et al., 2013). It is essential for a leader to conduct an evaluation of the activities openly. It helps the members to determine the direction of further actions in order to keep the coalition sustain (Clarke-McMullen, 2010; McNeish et al., 2019; Motley et al., 2013). In the literature, a stronger leadership can result in a greater sense of community (Peterson et al., 2019).

\section{Trust and cohesion}

The final studies included in this review showed that developing a trust and cohesive group is important for a coalition to be successful. A close relationship can lead to trust and sense of community in order to build a strong, productive, and sustain partnership (Brown et al., 2010; Clarke-McMullen, 2010; Doll et al., 2012; McNeish et al., 2019; Motley et al., 2013; Peterson et al., 2019; Reid et al., 2018). Furthermore, it is important to develop trust, not only in researchers - community members' relationship, but in the relationship among community members (McNeish et al., 2019). High trust between coalition members will create a mutual respect and generosity among the member (Reid et al., 2018). Consequently, the relationship is rewarding and the members feel comfortable to get involved (Clarke-McMullen, 2010), thus the partnership can sustain because of a deep sense of community (Motley et al., 2013; Reid et al., 2018). Several strategies are known to establish a cohesive coalition, including a consistent and respectful communication, collaborative action plans that engage the members to work in a cooperative rather than competitive way, and a strong commitment (Brown et al., 2010; Doll et al., 2012; McNeish et al., 2019). In the same way, other strategies were sharing ideas, common interests, concerns, experiences, vision, goals, and perspectives on the targeted issue (McNeish et al., 2019; Motley et al., 2013; Peterson et al., 2019). In addition, discussing the members' preconceived notions on their partner might be useful to relieve prejudice and establish a balance relationships (McNeish et al., 2019). An open and respectful communication creates a positive relationship and reduce potential conflicts between members (Clarke-McMullen, 2010; Doll et al., 2012). In the end, the trust and cohesion promote a strong and productive long-term relationship which sustain the coalition (Brown et al., 2010; Motley et al., 2013; Peterson et al., 2019; Reid et al., 2018). Apart from it, trust and cohesion should be built from the first day of the coalition (McNeish et al., 2019; Motley et al., 2013).

\section{Role clarity}

Partnering with members of specific expertise requires role clarity. Role clarity helps the coalition to recognize the expertise or network of its members, while allowing the coalition to have access to the targeted community (Anderson-Carpenter et al., 2017; 
Clarke-McMullen, 2010; Doll et al., 2012; McNeish et al., 2019; Motley et al., 2013; Reid et al., 2018). Role clarity helps members recognize their resources as well as the other member, which is helpful when they face a problem. The partnership would know who to ask for a support or assistance (Anderson-Carpenter et al., 2017; Reid et al., 2018). The clarity of the role also benefit the partners to align their expertise and their organization's mission with the goals of the coalition (McNeish et al., 2019; Reid et al., 2018). Moreover, the members would be able to actively engage in the coalition's activity when their role as well as the role of the others is clearly articulated (McNeish et al., 2019). Explicit roles and responsibilities help the coalition to design realistic plans as each member recognizes the expectations of their involvement (McNeish et al., 2019; Reid et al., 2018). With a clear and transparent role, the coalition members able to support the accountability of their partners (McNeish et al., 2019). At the same time, the members share their power with others (Anderson-Carpenter et al., 2017; Doll et al., 2012; Motley et al., 2013). It will create a positive working climate (Doll et al., 2012; Reid et al., 2018), as well as a positive attitudes towards the coalition (ClarkeMcMullen, 2010; Doll et al., 2012). On the other hand, role ambiguity may result in power struggles and confusion among members (Doll et al., 2012). As a consequence, negative outcomes might occur such as lack of motivation or disengagement of the members, and uncoordinated or ineffective implementation of coalition's programs (Doll et al., 2012).

\section{Norms and culture}

Apart from understanding the targeted issue, the coalition requires to understand the community itself (Doll et al., 2012; McNeish et al., 2019; Motley et al., 2013; Reed et al., 2014); to focus on the values and norms in the community, as well as the healthrelated issues (McNeish et al., 2019). It is vital for a coalition to be sensitive to culture from the very beginning of the coalition (Motley et al., 2013; Reed et al., 2014). As early as the recruitment of the members, identify the problem, plan the programs, and evaluate the activities. In understanding the problem, the coalition required to consider and understand the community's point of view by involving the community, as the member of the coalition (McNeish et al., 2019; Motley et al., 2013; Reed et al., 2014). In line with that, it is essential for a coalition to plan, build, implement, and evaluate the coalition's intervention with a cultural appropriateness mindset (Motley et al., 2013; Reed et al., 2014). The understanding of the cultural norms will assist the coalition to identify and implement methods to engage the community (Reed et al., 2014). Knowing the community can also be useful to find the appropriate way to shift the cultural norms related to the targeted health issue (Motley et al., 2013), including the understanding of the community's history in dealing with the problem beforehand (Motley et al., 2013; Reed et al., 2014). The cultural sensitive approach not only benefits the succession of the programs but also the sustainability of the coalition (Doll et al., 2012; McNeish et al., 2019; Motley et al., 2013; Reed et al., 2014). 


\section{Discussion}

This article presents findings from a literature review study regarding important aspects to be considered in applying a Community Coalition (CC) approach for health promotion intervention in urban areas. Based on the review of literature, this study highlights the importance of seven aspects in creating an effective and sustained partnerships among community coalitions. Specifically, the diverse expertise of the members, the clarity of the goal, the member's interest and motivation, the leadership, the trust and cohesion of the coalition members, the clarity of the members' roles, and understanding the norm and the culture of the target community.

Before explaining these findings, it is necessary to clarify that the present study focused on Community Coalition interventions, not on community-based intervention. This distinction needs to be conveyed due to the wide range of meanings of the term 'community-based'. The community-based intervention can be categorized under four types, which are: community as setting, community as a target, community as agent, and community as a resource (McLeroy, Norton, Kegler, Burdine, \& Sumaya, 2003). These four models present different views of the nature of community, the role of health providers and professionals, and the relevance of different outcomes (McLeroy et al., 2003).

In the present study, we pursed the review on Community Coalition interventions since the study is a follow-up to our previous studies which proposed a collaboration of health workers and community members to promote health in the community (Sokang et al., 2019b). The term 'Community Coalition' refers to a group of individuals representing diverse organizations, factions, or constituencies within the community who agree to work together to achieve a common goal (Feighery \& Rogers, 1990). The approach is different from other approaches due to a formal -businesslike arrangement between the members (Butterfoss \& Kegler, 2012; Feighery \& Rogers, 1990), while being fluid and flexible in implementation due to its sharing-governance and decisionmaking processes (N. B. Anderson, 2004; Huberty, Balluff, O’Dell, \& Peterson, 2010). In regards to the purpose, coalitions can address specific or broad topics, for example on obesity, improving walk routes or overall access to health services (N. B. Anderson, 2004).

Having addressed the terms, the following section of this paper discusses the results and implications of the study. In the result, we found that leadership, including a distinct governance structures and decision-making processes, was the key for a coalition to have a strong partnership. This finding is consistent with previous studies which emphasized the importance of shared governance and decision-making. By a sharing governance and decision-making, members have an overview of the coalition's strategies, plans, and how the programs will be evaluated (Huberty et al., 2010). Thus, members will be able to focus more on the targeted issues and working toward the coalition's goal (Bosma et al., 2010; Huberty et al., 2010). By contrast, if the members are not involved in the decision making process, they might reluctant to participate in the coalition (Gerassi \& Nichols, 
2018; Shortell et al., 2002). Hence, a good leader who understands the uniqueness of each member, and embrace the differences in a shared governance and decision-making structure is needed for a coalition to be able to achieve its goal.

We also found that developing trust and cohesion were essential to create a strong, productive and sustain partnerships. Similarly, previous research also found that a trust is a key element to achieve a cohesive and successful coalition (Bauermeister et al., 2017; Jagosh et al., 2015; Kegler, Hall, \& Kiser, 2010; Miller et al., 2017). Research showed that understanding partners' perspectives helps a coalition to get close relationships (Gerassi \& Nichols, 2018). This certainly is the intention of a coalition to make the members have a sense of community, thus consider themselves as part of the partnership. On the other hand, it is a challenge to nurture the relationship to a cohesiveness (Frass et al., 2014). Lack of trust is one of the challenges. Mistrust can create a barrier in a partnership, as it could trigger conflicts in the coalition (Jagosh et al., 2015; Kegler et al., 2010). Mistrust hampered the success of the program because it leads to an indifference among the members (Shortell et al., 2002). Our findings are in line with those of previous studies, which pointing the major role of trust and cohesion among partners to support a successful and sustain coalitions.

Member diversity was also found to be important. The diversity refers to not only involve professionals from different expertise, but also the involvement of community members and stakeholders. It is important for a coalition to be flexible in selecting community partners, as the members can provide different resources and unique skill sets to complement each other (Corbin et al., 2015; Cramer, Atwood, \& Stoner, 2006; Frass et al., 2014; McNeish et al., 2019). Previous studies highlighted the importance of working together with members from various backgrounds, especially to establish the changes in the community in policy (Mckinney, Fitzgerald, Winn, \& Babcock, 2017; Robertson-James et al., 2017). The current study echoed these findings. Diverse coalition members from different field of expertise have their specific skills and resources. These sources can be useful for the coalition, as it means the coalition has different facilities and broader networking.

Involving many people from different background undoubtedly means the coalition involves many interests. It is crucial to understand the perspectives of the member, as well as their priorities and interests (Robertson-James et al., 2017). To achieve this, the coalition needs to put a cooperation and compromise attitude as a culture; to share values with clear communication. When the partnership shares a common goal, the success of the coalition become the priority (Huberty et al., 2010; Laverack, 2014). Consequently, the coalition program will be effective, as shown by studies that diverse coalition membership influence the outcome of effectiveness (Butterfoss \& Kegler, 2009). Meanwhile, when the goals are incompatible, members might withdraw from the program. It could also affect the member's trust, and potentially influence the coalition's goal (Kreuter, Lezin, \& Young, 2010; Seitanidi \& Ryan, 2007). Therefore, the coalition needs to pay attention to the agendas of each member (Kegler et al., 2010), and clarifies the motives and interests from the start. 
Our result also highlighted the importance of the coalition's goal. The purpose and goal of the coalition must be decided from the start. It enables the coalition members to understand and follow the coalition's direction while complying their organizational goal (Cramer et al., 2006; Shortell et al., 2002). Other studies found that goal and purpose clarity affects the coalition's cohesiveness and effectiveness (Butterfoss, Goodman, \& Wandersman, 1996; Frass et al., 2014). A specific goal enables the members to develop clear strategies and action plans to achieve the goals or objectives of the partnership (Butterfoss et al., 1996; Gerassi \& Nichols, 2018). Furthermore, if the goals are successfully achieved, the members gain confidence and willingness to expand the program to reach a larger community (Shortell et al., 2002).

Additionally, the present study identified the importance of role clarity of the coalition members. Working together to achieve a common goal with many people from various expertise and backgrounds might lead to overlapping responsibility. Unclear roles can confuse the members and may lead to internal conflict and competition among the members (Butterfoss et al., 1996; Butterfoss \& Kegler, 2002). If that occurs, it potentially affects the coalition's overall process (Corbin et al., 2015; Seitanidi \& Ryan, 2007). On the other hand, a clear role description can help the members to focus on their roles and effort to address the targeted issue (Corbin et al., 2015; Flores et al., 2005). It promotes accountability of the member because they are clear about their responsibilities. Role clarity also promotes commitment because they have a contribution to the coalition.

Lastly, we also found that understanding a community's norms and culture is crucial. A coalition needs to be aware of the perception, the culture, and the values considered important by the community to ensure that the coalition gets insight into the community's experience (Bartholomew Eldredge et al., 2016). By involving the community members, the coalition will receive the perspective directly from the persons who experience it. Various studies have shown that alignment between norms and culture with interventions or programs will guarantee the sustainability of the coalition (Harper, Contreras, Bangi, \& Pedraza, 2003; Harper \& Salina, 2000; Suarez-Balcazar, Harper, \& Lewis, 2005). It empowers the community and helps the coalition to shift the cultural norms related to the targeted health issue (Kreuter \& McClure, 2004). In contrast, the intervention most likely to fail if the project is not culturally appropriate (Bartholomew Eldredge et al., 2016).

With regards to our finding, we found seven important aspects to be considered in forming a community coalition. These aspects are of valuable interest to be added in the existing definition and parameters that had been mentioned in table 6.17 of Bartholomew Eldredge et al., 2016 and table 13 of Kok et al., 2016. In the previous studies, forming a coalition defined as 'forming an alliance among individuals or organizations, during which they cooperate in joint action to reach a goal in their own self-interest' (Bartholomew Eldredge et al., 2016; Kok et al., 2016), while in the present study we found that it is crucial for the partnership to work together towards a commongoal. Although each coalition members have their own interests, they need to work as a team to achieve the common-goal of the coalition. The seven important aspects can be added to the parameters to form an effective and sustain coalition. 
There are limitations of our study that need to address. First, the coverage. Some articles on the topics might be published after the period of the present study and were not covered. Having said this, the reference databases used in this study should collect the majority of English language peer reviewed journal articles ( 60 articles from PubMed and 756 articles from PsycINFO) and we used snowballing as an additional search strategy (62 articles). Secondly, we only included articles that explicitly mention the use of the CC approach due to unclear boundaries between similar approaches, for example between the CC approach with community-partnered participatory research. While community-partnered participatory research focus on working together with community as part of the research team, the CC approach involves the targeted community in all stages of preparation, implementation, and evaluation of interventions. Thus, because of the different types of operationalization of similar concept we chose to only include the concepts that were clear and were in line with our criteria. Lastly, the qualitative method used in this study was able to capture the aspects that contribute to an effective and sustain a coalition. In the other hand, each partnership is unique and our study may not represent all community coalition approaches. Thereby, the generalizability of the study should be treated with caution.

\section{Conclusions}

Conducting a health promotion intervention in an urban area is a challenge, as the city provides both the source of healthcare and threats to the health. Therefore, a coalition needs to consider several aspects in order to achieve and sustain an effective coalition. In summary, it has been shown from this review that there are seven aspects considered important in applying the Community Coalition approach for health promotion intervention in urban areas, namely (1) the members' diversity, (2) the goal clarity, (3) the member's interest and motivation, (4) the leadership, (5) the trust and cohesion, (6) the role clarity, and (7) understanding the norm and the culture of the community. A note to notice is these aspects are not an independent aspect. The preparation, the implementation, and the process of achieving the aspects need to be done collectively by the coalition members. Furthermore, we propose the aspects found in this study to be added in the existing definition and parameters in Bartholomew Eldredge et al., 2016 and Kok et al., 2016. 

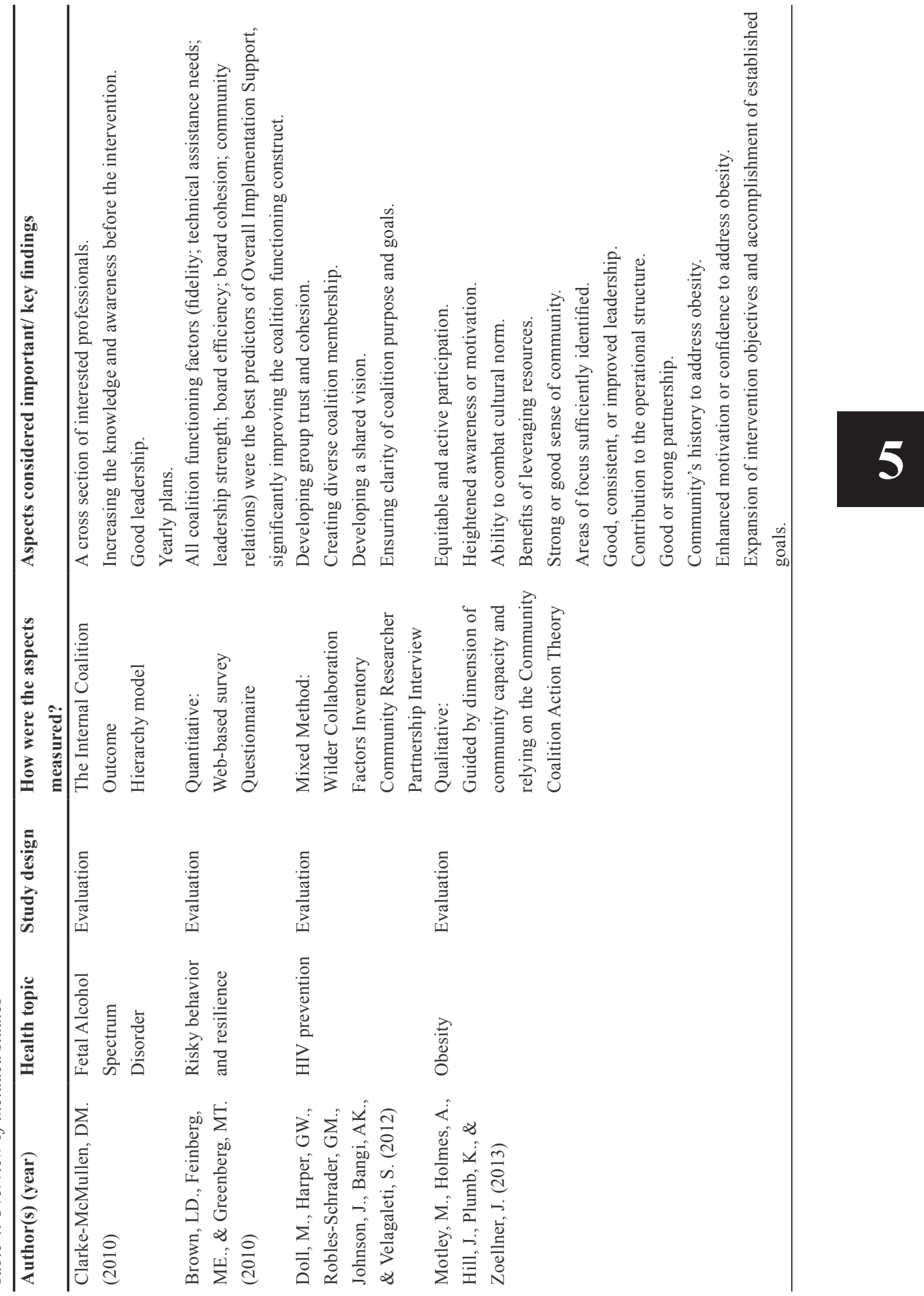


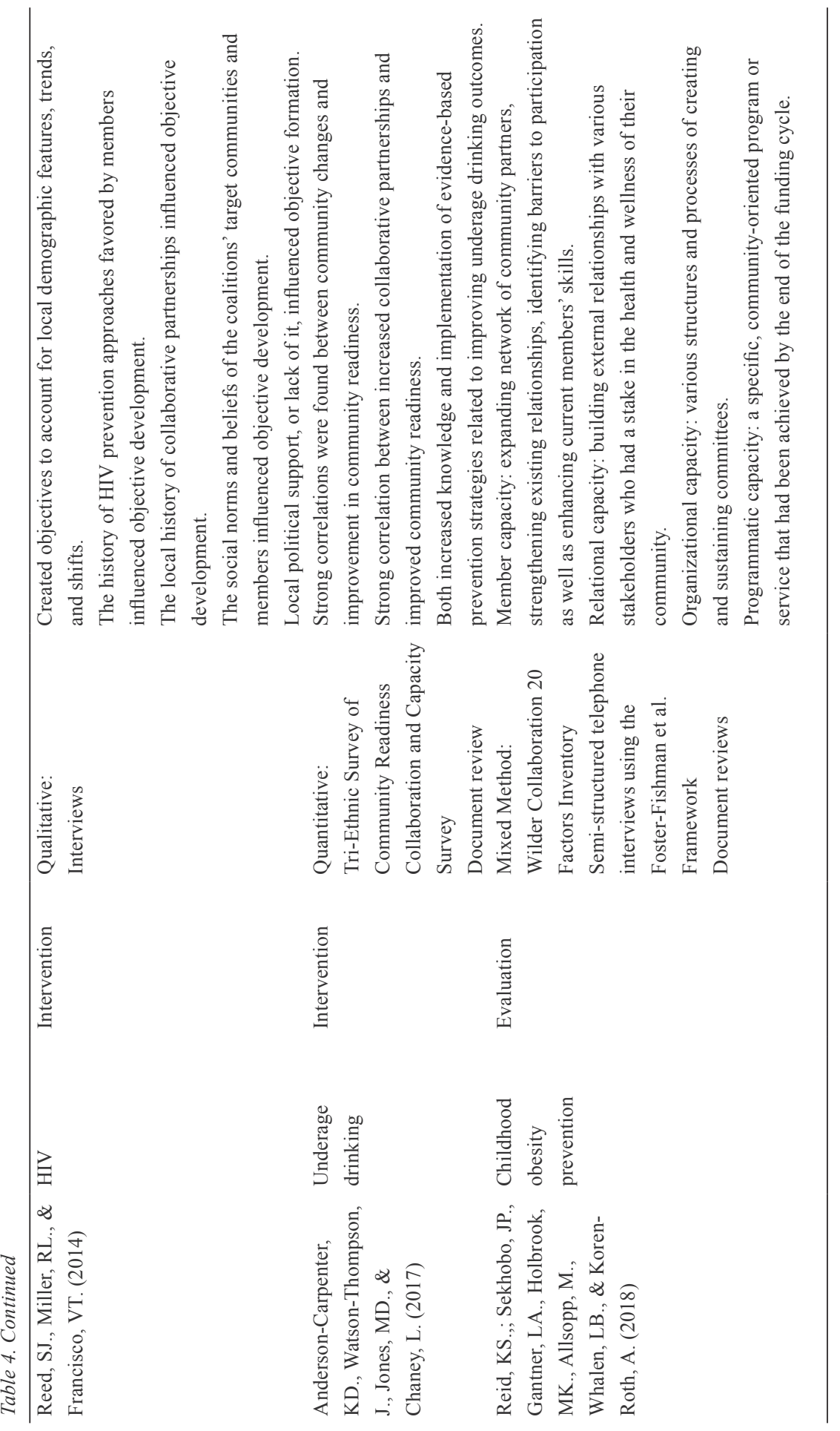




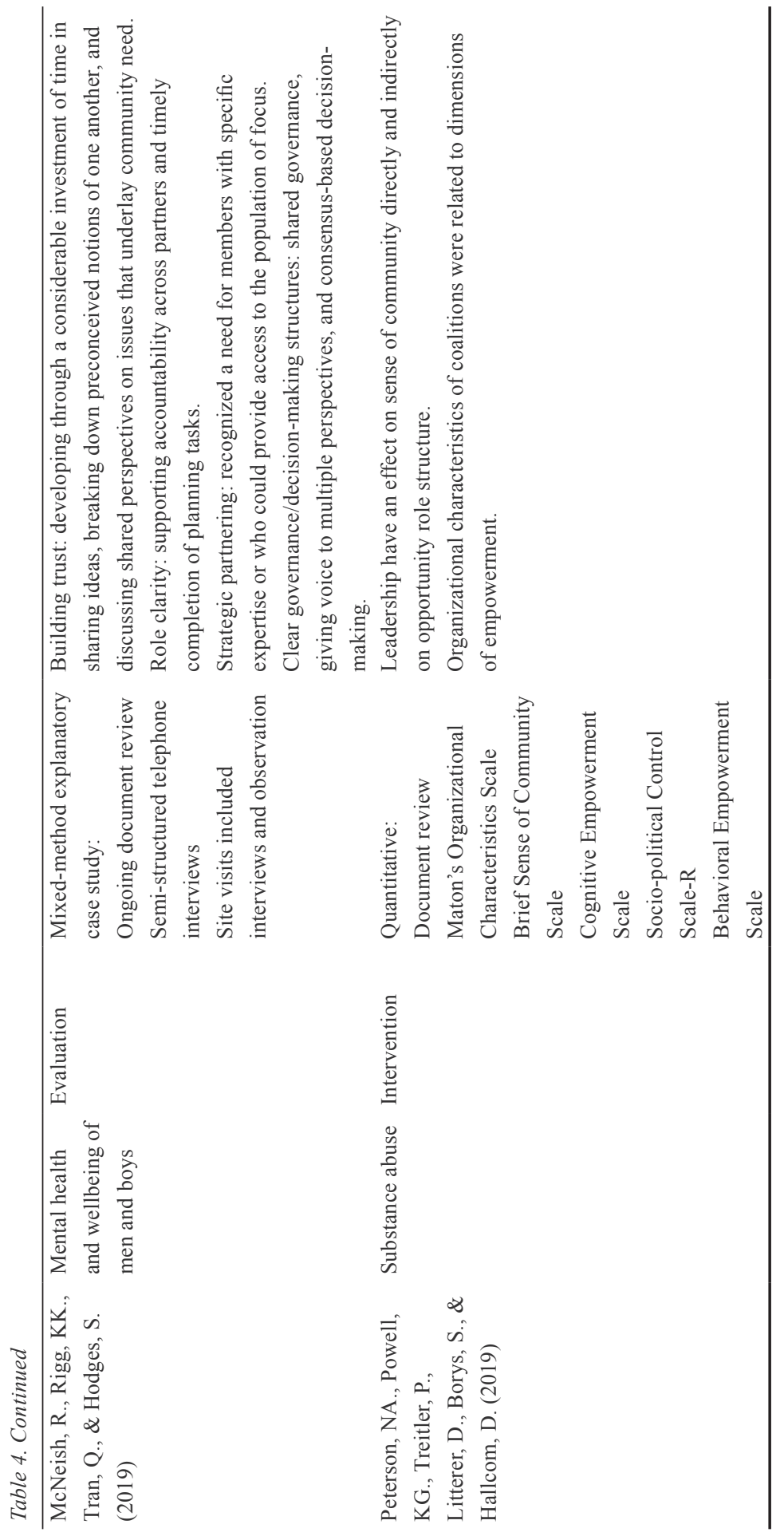




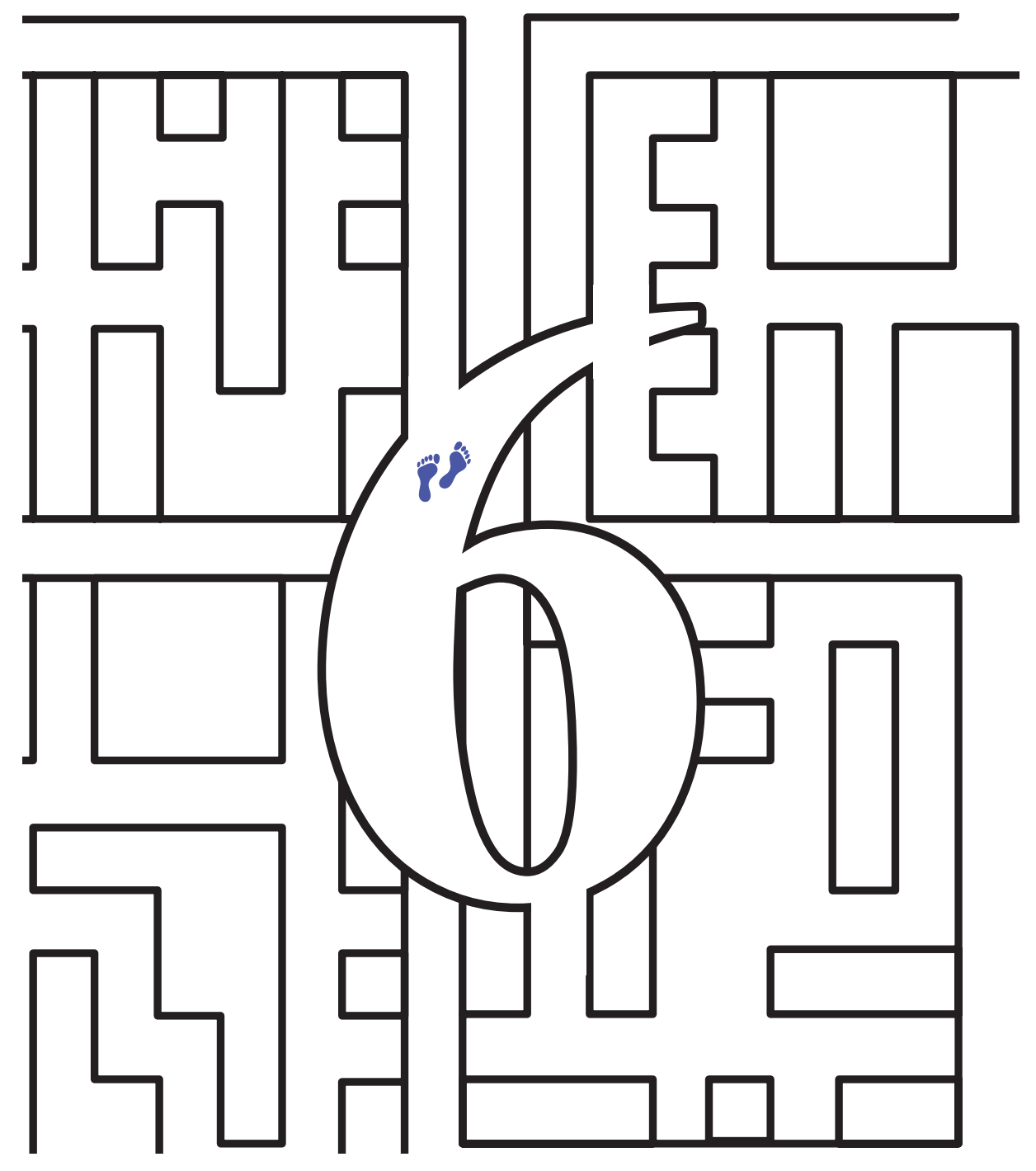




\section{Chapter 6.}

General Discussion 
Understanding and appreciating the cultural value of community will remain important for the foreseeable future. In this dissertation, we refer to culture as the characteristics and knowledge of a specific group of people that are learned, reinforced, shared, transmitted intergenerational, and reflected in community's values, norms, beliefs, practices, and patterns of communication, roles, and other social patterns. The understanding and appreciation of the community's cultural values can help health promotion practitioners and health promoters to understand the cause and effect of the health issues from community's perspectives, how to deal with said issues while considering community's cultural values, and use the community's cultural potencies to change their own behavior (Fertman et al., 2017; Green et al., 2019; Jongen et al., 2017). By being culturallysensitive, health promoters would understand how communities perceive their daily health-related behavior, how they perceive the health-related issues, how they handle the issues, as well as their expectation regarding the issue. Accordingly, health promoters would be able to communicate about health-related issues to the targeted community in the same language, literally and figuratively. In addition, understanding cultural values helps health practitioners to become conscious about their roles in the targeted community (Bartholomew Eldredge et al., 2016; Gottwald \& Goodman-Brown, 2012). A health promotion intervention would be successful and sustainable if the health promoters understand and make use of the cultural values of the targeted community when they implement their intervention (Fertman et al., 2017; Gottwald \& GoodmanBrown, 2012; Jongen et al., 2017).

Moreover, the biomedical perspective on health promotion has been one of the most prominent ones in the literature, although this perspective pays insufficient attention to the cultural values of the targeted community (Setiyawati et al., 2014). The biomedical perspective sees health and sickness mostly as a result of, for example, sanitary/hygiene and infection (Kahissay et al., 2017). On the contrary, in developing-countries such as Indonesia, the society views health and sickness in a much more complex way, because they perceive health from multiple points of view, which includes cultural and religious aspects (Conti, 2018; Sokang et al., 2019a; Whitehead et al., 2016). In a society where health and sickness are interpreted from a multidimensional perspective, health-related behavior, including health-seeking behavior, is viewed differently compared to a society that relies on biomedical perspective (Conti, 2018; Nurhayati \& Widowati, 2017).

Studies have also shown that a cultural sensitivity approach links to better patients' satisfaction (Betancourt, Green, \& Carrillo, 2002; Foronda, 2008; Phillips, Chiriboga, \& Jang, 2012), improves health outcomes (Betancourt, Green, Carrillo, \& Park, 2005; Thom \& Tirado, 2006; US Department of Health and Human Services - Office of Minority Health, 2001), and has been proven to be effective for health promotion in a group of adults with a low level of literacy (Winkleby et al., 1997) and working class adults from multi-ethnic backgrounds (Sorensen et al., 2005). Furthermore, implementing a culturally-sensitive approach has been associated with better treatment adherence and an increase in the community's engagement in health promoting behaviors, which consequently improves the community's health outcomes (Tucker, Arthur, Roncoroni, 
Wall, \& Sanchez, 2015). Additionally, culturally-appropriate approaches protect the targeted community. Communities with strong cultural backgrounds tend to have strong traditional healing cultures. With a culturally-sensitive approach, health promoters would be able to recognize herbal remedies that are used in the communities and perhaps be able to adjust the modern medicine to meet the needs of the community to avoid serious complications (Young \& Guo, 2016). Thus, in order to address health issues comprehensively, it is crucial for health promoters to understand the cultural values of the targeted community. This way, health promotion will be properly delivered to the targeted community (Bartholomew Eldredge et al., 2016; Green et al., 2019; Jongen et al., 2017).

Considering the prominent biomedical as opposed to the community-centered perspective in health promotion and the importance of cultural values, the overall aim of this dissertation is, with explorative studies, to understand the important values for health and health promotion by community members from Jakarta. This includes, but is not limited to, the health workers, while paying attention to the cultural context of the issues. The research questions of this dissertation are: 1) What do health and sickness actually mean to Jakartans (i.e., the citizens of Jakarta city)? 2) How do health care users in Jakarta perceive the quality of the provided health services? 3) What are the challenges and obstacles for conducting health promotion programs in Jakarta from the perspective of the health workers? 4) What are the aspects that are considered to be important in applying a Community Coalition approach for health promotion intervention in urban areas? A Community Coalition (CC) is a group of individuals from diverse organizations, or constituencies within the targeted community who agree to work as a team to achieve a common goal (Feighery \& Rogers, 1990).

The present chapter summarizes the main findings of the studies presented in Chapter $2,3,4$, and 5 , followed by a discussion of the strengths and limitations of the studies, as well as implications for future research and practice of health promotion interventions in Jakarta. During the project, we used qualitative research methods with open-ended questions, focus group discussions (FGDs), and a literature review study.

\section{Main findings}

Chapter 2 was designed to answer the first research question by describing the meaning of health and sickness according to Jakartans. Using an open-ended survey, the study showed that Jakartans perceived their health and sickness not only as a single condition (e.g., in relation to physical health), but also as a combination of many conditions (e.g., in relations to physical and psychological health). As a single condition, health was perceived as a physical condition, a psychological condition, a spiritual condition, a capability to carry out daily activities, or a healthy lifestyle. Meanwhile, sickness as a single condition identified as a physical condition, a psychological condition, abnormal circumstances or bad situations, a spiritual condition, or an inability to carry out daily 
activities. As a combination of many conditions, health was perceived as, for example, a combination of physical, psychological, and spiritual conditions; or a combination of physical, psychological, and healthy lifestyle aspects, etc. In Chapter 2, we discussed how the perceptions of health and sickness might influence the health-related behaviors in the everyday life of Jakartans, including how they treat their illnesses. In addition, we also discussed how the current health care system in Jakarta might not accommodate the multidimensional perspectives of Jakartans regarding their health and sickness.

These findings re-emphasized the importance of recognizing the community's perception of health-related issues, particularly on health and sickness themselves. The discussion presented in the second chapter was in line with that of previous studies that showed people's perceptions on health and sickness were linked to their daily healthrelated behavior (Raza et al., 2016; Vaughn et al., 2009). Furthermore, our study discussed how socio-economic conditions and governmental policies influenced people's healthrelated behaviors. For example, in developed countries, government regulations required companies to continue pay the salary for employees who take a sick leave, e.g. in the Netherlands up to five days for recovering from flu (Enneman, 2014; Jongepier, 2017; Ministerie van Sociale Zaken en Werkgelegenheid, 2011). However, in Jakarta, most employees (close to $65 \%$ of the Jakartans) are part of a less-structured working system in which they receive daily payments. It means that these employees do not get their daily wage when they take a sick leave (Simone, 2015). As a result, the Jakartans tend to do self-treatment and still go to work despite being (clinically/physically) sick (Sokang et al., 2019a). Additionally, to address Jakartans' health-seeking behavior, it is important for health practitioners to pay more attention to the use of traditional medicine; not to eliminate the use but to understand its benefit for the community. At the same time, by understand the Jakartans' use of traditional medicine, health providers would understand their needs and beliefs with regard to their health-seeking behavior. Communities use traditional medicines because they believe in the effectiveness of these medicines (Nurhayati \& Widowati, 2017; Pengpid \& Peltzer, 2018) and community members may become resistant to the use of modern medicines. This becomes a challenge to behavior changes. Integrating modern and traditional treatments can be one of the ways to allow people to use traditional treatment/medicine while being supervised by registered doctor or health practitioner (Mendoza, 2009; Pengpid \& Peltzer, 2018; Sokang et al., 2019a)

Chapter 3 focused on the second research question by identifying how health care users in Jakarta perceived the quality of health care services. Using FGDs, we found that Jakartans viewed community health centers (CHCs) negatively, and they preferred private clinics and hospitals over the government-mandated CHCs. CHCs were viewed negatively due to their unfriendly staff, long waiting period, short opening hours, and crowded visitors. Participants also highlighted the low quality of CHCs, including the low quality of medicine and medical equipment, as well as the need for psychological services at CHCs. However, Jakartans had a more positive attitude toward the affordability of CHCs. Lastly, this study also revealed the motivations for 
using self- and traditional treatments by Jakartans before visiting health care services. Together, the findings in Chapter 3 shed light on how the government and health care providers in Jakarta could facilitate health-related needs of Jakartans by implementing better health care services. In the discussion, we recommend the government, health care providers, and policy-makers to improve the hospitality of $\mathrm{CHC}$ staff members and the quality of CHCs' medicine and medical equipment, as well as to provide psychological services at $\mathrm{CHCs}$.

The results of this study introduce a gap between health care services that are provided with health care services that are needed in Chapter 2 . The community perceives their health and sickness from multiple perspectives while health care providers focused more on the biomedical perspective (Conti, 2018; Peltzer \& Machleidt, 1992; Richter, 2003). Moreover, the poor services at CHC makes community members to become reluctant to go to CHCs (Kayral, 2014; Mitropoulos et al., 2018; Senitan et al., 2018). Research has shown that users of health services, in this case the patient and the family, prefer short waiting hours (Akhter et al., 2015; Alijanzadeh et al., 2016); low cost health care services (European Patients' Forum, 2017); and modern technology (Khamis \& Njau, 2016). The use of modern and appropriate medical equipment increases users' confidence in the quality of health services (European Patients' Forum, 2017; Khamis $\&$ Njau, 2016). Similarly, users trust a friendly staff -who are perceived as caring- to take care of their family or themselves (European Patients' Forum, 2017). However, health care services in Jakarta did not provide such services and mainly focused on only physical conditions (Balog, 2005; Conti, 2018; Gottwald \& Goodman-Brown, 2012; Rheenen, 1998). For example, psychological services are not yet available in most CHCs in Jakarta (Carina, 2017), whereas based on the results in chapter 3, Jakartans feel the need for psychological services.

Chapter 4 addressed the third research question by exploring how general practitioners (GP) at the CHCs perceived the challenges and obstacles of conducting health promotions in communities in Jakarta. We used FGDs to probe how GP-interns perceived the current health promotion programs conducted by CHCs in Jakarta. The result showed that the GP-interns had a negative perspective toward the implementation of health promotion program at CHCs. The main reason was that they considered CHCs to be understaffed, which led to a limited time the doctors had to treat and educate the patients about their disease or illness, not to mention the time to educate community members on general health-related issues. The lack of staff also resulted in a shortage of time for health workers to identify and explore health problems that are particular to certain communities in Jakarta. Consequently, health promotion was only delivered in public seminars on health, which are often too general and target unsuitable group members of the community. Furthermore, the challenges also came from the Jakartans as the target of health promotion programs. Their lack of awareness with regard to a healthy lifestyle is one of those challenges. For example, Jakartans tend to underestimate the severity of their illness by using self- and traditional treatments, 
instead of visiting CHCs and getting treatment by GPs. To overcome these challenges, the participants of this study proposed that the local government in Jakarta and the policy makers to invite community leaders, clinical psychologists, and religious leaders to take part and or collaborate in the health promotion programs at CHCs as facilitators.

The result of our FGDs also revealed the struggle of health workers, especially the GPs, in conducting health promotion, which runs in complement to the findings presented in Chapter 3. Specifically, the lack of understanding on the needs and healthrelated behaviors of the community might originate from the lack of time and information health workers had to implement ideal health promotion programs. For example, the negative perspective of traditional treatments and medicines causes a negative attitude by the GPs toward patients who used traditional treatments or medicines. Whereas, the community considered the traditional treatment and medicine as part of their tradition and as having cultural values (Nurhayati \& Widowati, 2017; WHO, 2013). Besides, health workers tend to limit their approach on the healing and recovery processes with an emphasis on the curative-rehabilitative services (Australia - Indonesia Partnership for Health Systems Strengthening, 2016). In contrast, in Indonesia, the Minister of Health's decree number 585/Menkes/SK/V/2007 mandates that health promotion programs should highlight the efforts to learn from, by, for and with the community. Thus, empowering the community to be able to help themselves and to contribute to the developing community-based activities, which are in line with their local socio-cultural conditions (Ministry of Health Republic of Indonesia, 2007).

In increasing the participation of multiple parties in the development of health promotion programs, the Indonesian government's efforts to involve health cadres/ volunteers can be considered to be commendable. These cadres help health workers to approach communities (Caturiningsih et al., 2016; Qusna, 2016; Tristantia \& Khoirunnisa, 2018). Health cadres/volunteers are community members who are chosen by community members and work voluntarily to maintain the health level of a certain community. They are trained and work together with $\mathrm{CHCs}$, and are under the supervision of the village office (Indonesian: kelurahan). In spite of their contributions, health cadres have been shown to have limited knowledge and skills to communicate effectively with community members (Berman, 1984; Nazri et al., 2016). Accordingly, to conduct effective and sustainable programs, health workers need to collaborate with other parties -such as health cadres- to build and implement health promotion programs in the community. One of the approaches that might be an ideal approach is the Community Coalition (CC) approach, which we elaborated on in chapter 5.

Chapter 5 covered the fourth research questions by synthesizing important aspects of the possible applications of the CC approach for health promotion intervention in urban areas. This study is a follow-up study to respond to a suggestion of the study described in Chapter 4. There, the scope of our approach was limited to urban areas. Here, we searched and analyzed existing studies that used the $\mathrm{CC}$ approach in a literature review. The final literature review included nine records, which came from 816 articles 
found in PubMed and PsycINFO databases, and using the snowballing approach based on the articles found in the databases. The results revealed seven aspects, which sometimes overlap, and which are considered important for the application of the CC approach for health promotion intervention in urban areas, namely: (1) the diverse expertise of the members, (2) the clarity of the goal, (3) the member's interest and motivation, (4) the leadership, (5) the trust and cohesion of the coalition member, (6) the clarity of the member's roles, and (7) understanding the norm and the culture of the target community. Coalition members should work hand in hand to prepare, implement, and attain these aspects. Thus, these seven aspects help health promoters create an effective and sustainable coalition while paying attention to needs of community.

Our review suggests that conducting health promotion effectively and sustainably requires collaboration from various parties, as health promotion includes a multisector approach (Bartholomew Eldredge et al., 2016). This multisector approach is in line with the spirit of $\mathrm{CC}$ approach, which involves parties who agree to work together toward a common goal (Anderson-Carpenter et al., 2017; Doll et al., 2012; Feighery \& Rogers, 1990). The CC approach might be appropriate for Jakarta's community as health workers benefit from the presence of health cadres/volunteers (Caturiningsih et al., 2016; Qusna, 2016; Tristantia \& Khoirunnisa, 2018). However, the relationship between health workers and health cadres in Jakarta is still a top-down relationship. It means health cadres were seen as 'helpers' not as partners (Basu, 2010; Cross, Davis, \& O'Neil, 2017; Tunas \& Peresthu, 2010). This top-down paradigm needs to be changed into a coalition perspective so that health cadres/volunteers and other stakeholders are involved in every steps of health promotion programs equally. Furthermore, the sharing-governance and decision-making processes in the $\mathrm{CC}$ approach is also in line with the Indonesian values of deliberation and consensus (Indonesian: musyawarah dan mufakat), which are a traditional decision-making principle in Indonesia. Lastly, by implementing the $\mathrm{CC}$ approach, health workers would be able to pay more attention to the cultural context of health-related issues, thereby fulfilling the needs of the targeted community.

\section{Methodological reflections and implications}

The focus of this dissertation is on a need assessment in Jakarta's community, and it mostly uses a qualitative approach in data collection. As in many other studies, this project has its own strengths and weaknesses. Some of the strengths are, firstly, that the dissertation provides empirical evidence that suggests the importance of considering the cultural values of the targeted community in the implementation of health promotion programs. The studies gave detailed insight into the perspectives of Jakartans, both the community members and the health workers, of health and health promotion, which are in stark contrast to the approaches of available studies that tend to discuss interventions 
and health promotion programs in the context of a specific disease or health-related issue. To the best of our knowledge, there have been no international peer reviewed publications (especially related to Chapters 2 and 3 ) regarding the perspectives of health and sickness and how community members evaluate primary health care services in Indonesia. This absence of reputable publications might lead to the misunderstanding of the concept of health in Jakartans during the development of interventions and health promotion programs.

Our findings imply that health promotion researchers and health promoters need to consider to use deep structure dimensions of cultural sensitivity in conducting health promotion interventions; not merely translating the programs into the local language, but also understanding the cultural, historical, and social aspects of the targeted community. This deep structure not only gives an opportunity to respect the core cultural values of the targeted community, but also to use the values as resources to improve health (Resnicow et al., 1999, 2000). Secondly, the needs of community can be expressed appropriately using a qualitative approach. That approach is proper to explore preliminary data, to uncover the perspectives and needs of Jakartans, due to the limited health promotion research focused on Jakartans' points of view. Thirdly, this research maintains the reliability of the data by minimizing personal biases. The data collected was coded separately by four research assistants, and the results were discussed together under the supervision of the primary investigator. The results were then discussed with the two supervisors of the primary investigator in order to further reduce research biases. Lastly, the data included responses from different ages and educational levels of participants. Thus, providing the benefit of a wide range of perspectives.

On the other hand, this dissertation also has several shortcomings. Our primarily qualitative approach provided deep insights in the beliefs and concepts of health and health promotion among the Jakarta communities. However, these insights could not be quantified and data triangulation with quantitative data could not be implemented. Hence, the transferability of the results is limited. In order to transfer our result to a larger population, the next step proposed is to investigate the community perspective of health and health promotion in larger multisite studies with quantitative procedures or mixed methods.

\section{Practical Implications and recommendations}

In order to improve the community's health level, it is crucial for community's stakeholders, including researchers, health providers, and policy-makers to work together as a team. However, creating an effective partnership can be an additional challenge on top of the complexity of the present health issues in the communities. A proper plan needs to be developed, even prior to the gathering of team members to maintain the fundamental elements of health promotion in community settings, i.e. the empowerment and community participation aspect (Green et al., 2019; Poland et 
al., 2009). Therefore, there are suggestions emerging from this dissertation, along with potential studies that are required to improve the understanding on the health-related needs, while respecting community's cultural value, especially in Jakarta, Indonesia.

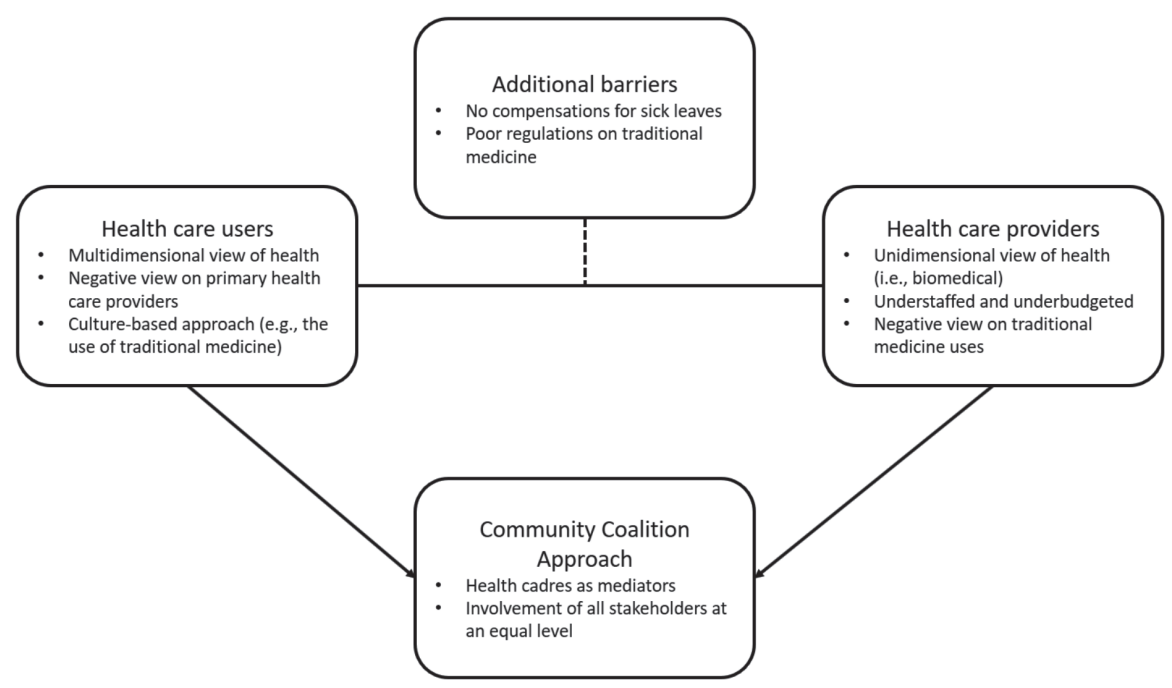

Figure 2. Logic model of health promotion gap in Jakarta

The outcomes of this dissertation strengthen our belief to involve the members of the community and the stakeholders as part of the health promotion team. The involvement of community members is essential in order to conduct health promotion interventions based on the cultural values of the community. Such an intervention would be able to facilitate the needs of the community members to remain performing their traditions, including in health-related matters. Following the $\mathrm{CC}$ approach (Butterfoss et al., 1993; Butterfoss \& Kegler, 2002; Feighery \& Rogers, 1990) and utilising the Intervention Mapping (IM) framework (Bartholomew Eldredge et al., 2016), the next section explains future plans to address the logic model of health promotion gap in Jakarta as shown in Figure 3.

In the near future, we would disseminate the results of this dissertation to Jakarta stakeholders, such as the Jakarta government, especially the Grogol Petamburan subdistrict officials, CHCs, health practitioners, health researchers, health promoters, and the member of Jakarta communities, including but not limited to religious leaders and health cadres/volunteers. As part of the dissemination, we will explain that purely biomedical approach is unsuitable for Jakartans, as they perceive health and sickness from various aspects. Therefore, it is important to pay more attention to health and health promotion in general. The critique formulated in this dissertation will be presented to the Jakarta stakeholders that they underestimate the public's perspectives regarding health and health promotion, and mainly focus on specific diseases. 
The next step is to establish and work with a planning group, as one of the Step 1's tasks of the IM framework (Bartholomew Eldredge et al., 2016). We would invite the Jakarta stakeholders to improve health and health promotion programs in Jakarta by forming a coalition. We would invite sub-district (Indonesian: kecamatan) and village (Indonesian: kelurahan) officials as representation of Jakarta government and policy makers, CHC staff e.g. GPs, nurses, and midwives, clinical psychologists, health researchers from universities, head of community associations (Indonesian: rukun warga $R W$ ), head of neighborhood associations (Indonesian: rukun tetangga, $R T$ ), health cadres/volunteers, religious leaders, and community members. Hereafter, the Jakarta stakeholders will be referred to as the coalition members. When all have agreed and a coalition formed, the coalition members would discuss the goals of the coalition while clarify the interest and motivation of the members, leadership and staffing as well as the structure of the coalition to clarify the member's roles. Besides, as part of the task in the IM framework, the discussion on goals, interest and motivation, leadership, etc. are some important aspects in the CC approach as stated in Chapter 5.

In regard to the goals, the coalition goals can be divided into sections, such as shortterm, medium-term and long-term goals. Short-term goals would extend the first step of the IM framework to assess a wider range of participants using questionnaires or surveys that are tailored based on the initial research in this dissertation. The studies can addresses topics such as a) an evaluation on health care facilities provided in Jakarta; b) an evaluation of health promotion activities performed by $\mathrm{CHC}$; c) evaluations of CHCs' performance, both from the users and the community, as well as the staff members of the CHCs. The results would be useful to provide rich information and feedback to establish the present result.

The next step proposed is to generate logic model of change by developing insights of appropriate health promotion interventions for Jakartans. In this stage, the team combines evidence, both from scientific literature and the opinion and experience of community members, as well as from other stakeholders (Bartholomew Eldredge et al., 2016). In the coalition, we would obtain scientific literature from universities/health promotion researchers, and empirical-evidence from the previous step's studies. The goal is to create a blueprint of interventions based on the share-decisions among the coalition members that fit the needs, beliefs, and values of Jakartans. In the blueprint, the coalition explains the logic of the model of targeted behavioral and environmental change, by creating matrices of change objectives. The matrices contain detailed explanations of determinants of the targeted behaviors, and performance objectives for expected behavioral and environmental outcomes (Bartholomew Eldredge et al., 2016). The intervention blueprint would be beneficial as a solid proposal to obtain funds.

The next step is to apply for funding to build and implement the blueprint. There are few options for funding resources. First, from Indonesian government, especially the Ministry of Health of the Republic of Indonesia. The proposal can be submitted by sub-district and village officials as coalition members. The second possible way to gain funding is to submit the proposal to the Health Office of Jakarta Municipality. 
The CHCs staff would able to submit the proposal as member of the coalition. The third option is to apply for funding from the Ministry of Research and Technology/ National Research and Innovation Agency of the Republic of Indonesia. The proposal can be submitted as community service programs of the researchers' university. The other options to gain funding is from corporate social responsibility funding, nongovernmental organizations, or crow-funding options.

When the coalition gets funding for the intervention, the coalition would focus on the medium-term goals that is creating the intervention's program design of the abovementioned blueprint. In the IM framework, this stage of mapping an intervention is included in the third step, of which the tasks are: generate program themes, scope, and sequence, design practical application to deliver change methods based on theory and empirical-evidence behavior change methods (Bartholomew Eldredge et al., 2016).

Based on our present results, CHCs experience challenges in performing their main tasks which are health promotion and prevention. Thus, the first scope of the intervention would be establishing a special 'field' team with the task is to focus on health promotion in community, as suggested by our participants in the fourth chapter of this dissertation, and has been studied on Chapter 5. Additionally, the plan to establish a special team is in line with the Act of the Republic of Indonesia number 36 of 2009 about Health, the Health Ministry Decree Number 128 of 2004 about Basic Policies of Community Health Centers, and the regulation of the Indonesian Ministry of Health number 75 of 2014 which emphasize cross-sectoral networking to improve health in the community (Ministry of Health Republic of Indonesia, 2004, 2014a; The President of the Republic of Indonesia, 2009). The special team members including e.g. a clinical psychologist, health cadres/volunteers, religious leader, and a medical staff such as a doctor, nurse, or a midwife. Regarding religious leaders, we are aware that there are controversial topics in faith and health care, such as immunization, harm reduction and HIV, family planning, and abortion and artificial reproductive technology (Tomkins et al., 2015). For that reason, the special team members would receive training and fulfill specific roles that are in line with their own interest and motivation.

The second component of the intervention that we would address is to reinforce the implementation of the Indonesian Health Minister' regulation number 44 of 2018 regarding the involvement of hospitals in health promotion efforts (Ministry of Health Republic of Indonesia, 2018c). Even though the CHC is the frontline institution, health promotion is a complex issue. By reinforcing the implementation of these regulations, the CHC's tasks might be more practical. Thus, the CHC staff members are able to get better engaged in the community. With the special team, and assistance from the hospital, CHC staff would provide guidance and education to the community about health, including the use of self-and traditional treatments. The coalition may assist the government in working together with hospital by inviting hospitals to get involved in the coalition.

The other component to be addressed is to disseminate the regulation regarding Indonesia's traditional medicines in the Indonesian Health Minister's regulation number 
HK.01.07/MENKES/187/2017 (Ministry of Health Republic of Indonesia, 2017c). The regulation underlines how to use these traditional medicines by following the instructions, without neglecting GP's treatments or prescriptions, and consulting about their use with professional health workers. However, our results in Chapter 4 indicated that GP's tend to have a negative view toward traditional medicine. Therefore, we propose that the Jakarta government, CHCs and hospitals disseminate the regulations to health care workers, for example via universities that have medical, nursing, and/or midwifery departments. The dissemination would help the health care workers to have a positive attitude toward traditional medicine. The coalition would support the Indonesian government to disseminate the regulations and policies to Jakarta communities through these partnerships. This way, the information will be delivered easily, as the coalition members have their own networks. On the other hand, by utilizing the collaboration, the Jakarta government would overcome the lack of manpower at CHCs.

For the long-term goals, the coalition would focus on step 4, 5, and 6 of the IM framework, which result in program materials production, program implementation plan, and evaluation plan. The implementation of steps 4, 5, and 6 will follow the development in achieving short- and medium- term goals of the coalition. On the other hand, even though there are six steps in the IM frameworks, the steps are iterative rather than a linear process. In the future, we hope that the implementation of health promotion interventions can result in health policies and regulations that are appropriate to meet the challenges of health care providers and the needs of the Jakarta communities.

\section{Conclusions}

The results of this dissertation provided insight that the people of Jakarta city believe that their health and illness conditions are influenced by many aspects of life, including the spiritual aspects. However, they could not find an appropriate and satisfied service when visiting health services provided, especially not in the CHCs. As a way out, they choose to use self-and traditional medicines to treat themselves. On the other hand, health workers in the $\mathrm{CHC}$ are too understaffed to be able to properly approach and educate the community about health, and health promotion, including the use of self-and traditional treatment. Therefore, this dissertation discussed suggestions to be proposed to the community's stakeholders, including health researchers, government, health providers, and policy-makers. Lastly, in implementing these suggestions, stakeholders are expected to use theories and evidence, while paying attention to cultural values of the targeted community. 


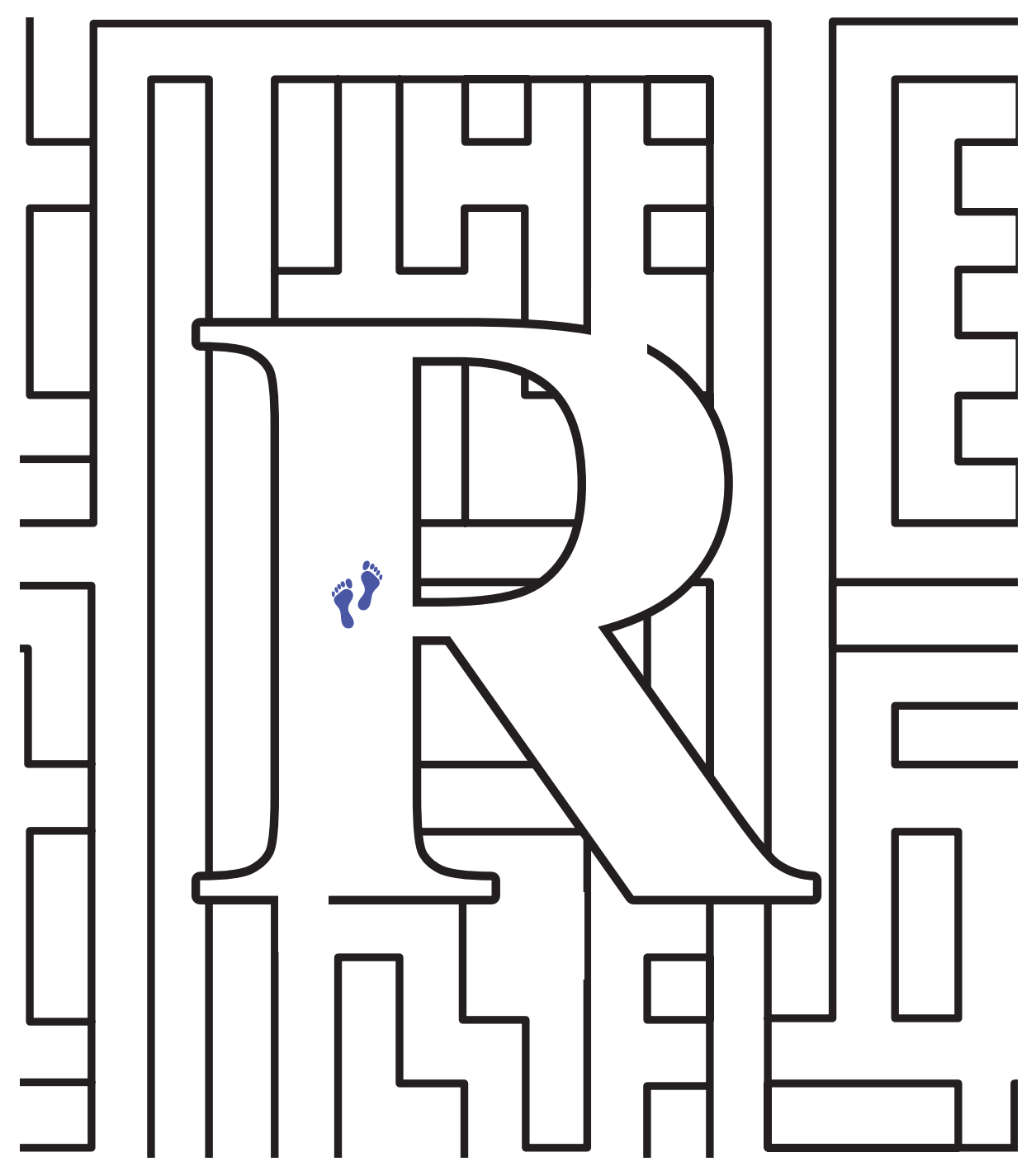




\section{References}




\section{References}

Agustina, R., Dartanto, T., Sitompul, R., Susiloretni, K. A., Suparmi, Achadi, E. L., ... Khusun, H. (2019). Universal health coverage in Indonesia: concept, progress, and challenges. The Lancet, 393(10166), 75-102. https://doi.org/10.1016/S0140-6736(18)31647-7

Akhter, N., Sushil, J., Howlader, R., Sultana, N., Ishaq, F., Ziaul, M., ... Rahman, T. (2015). Health Care Seeking Behavior of Slum-Dwellers in Dhaka City: Results of a Household Survey. Dhaka. Retrieved from http:// www.searo.who.int/bangladesh/publications/health_care_seeking_slum_dwellers.pdf

Aldiabat, K. M., \& Le Navenec, C.-L. (2018). Data Saturation: The Mysterious Step in Grounded Theory Method. The Qualitative Report, 23(1), 245-261. Retrieved from https://nsuworks.nova.edu/tqr/vol23/iss1/18/

Alijanzadeh, M., Moosaniaye Zare, S. A., Rajaee, R., Mousavi Fard, S. M. A., Asefzadeh, S., Alijanzadeh, M., \& Gholami, S. (2016). Comparison Quality of Health Services between Public and Private Providers: The Iranian People's Perspective. Electronic Physician, 8(9), 2935-2941. https://doi.org/10.19082/2935

Amato, K., Park, E., \& Nigg, C. R. (2016). Prioritizing multiple health behavior change research topics: expert opinions in behavior change science. Translational Behavioral Medicine, 6(2), 220-227. https://doi. org/10.1007/s13142-015-0381-5

Amegbor, P. M. (2017). Understanding usage and preference for health care therapies in a Ghanaian context: A pluralistic perspective. Norsk Geografisk Tidsskrift, 71(5), 288-300. https://doi.org/10.1080/00291951.2017 .1340908

Ammendolia, C., Cassidy, D., Steensta, I., Soklaridis, S., Boyle, E., Eng, S., ... Côté, P. (2009). Designing a workplace return-to-work program for occupational low back pain: an intervention mapping approach. $B M C$ Musculoskeletal Disorders, 10, 65. https://doi.org/10.1186/1471-2474-10-65

Anderson-Carpenter, K. D., Watson-Thompson, J., Jones, M. D., \& Chaney, L. (2017). Improving community readiness for change through coalition capacity building: Evidence from a multisite intervention. Journal of Community Psychology, 45(4), 486-499. https://doi.org/10.1002/jcop.21860

Anderson, C. (2010). Presenting and evaluating qualitative research. American Journal of Pharmaceutical Education, 74(8). https://doi.org/10.5688/aj7408141

Anderson, N. B. (Ed.). (2004). Encyclopedia of health and behavior (1st ed.). Thousand Oaks, California: SAGE Publications, Inc.

Apsari, R., Pardosi, R. R., Alifah, S., Windiyarti, N., Nurhayati, Pamujiyanti, T., ... Ningsih, D. S. (2016). Welfare Indicator of DKI Jakarta 2016. Badan Pusat Statistik Provinsi DKI Jakarta. Jakarta: Badan Pusat Statistik Provinsi DKI Jakarta. Retrieved from https://jakarta.bps.go.id/index.php/publikasi/ index?Publikasi $\% 5$ BtahunJudul $\% 5 \mathrm{D}=2016 \&$ Publikasi $\% 5$ BkataKunci $\% 5 \mathrm{D}=\& y \mathrm{t} 0=$ Tampilkan

Arthen, D. (2016, May 31). The fitness lifestyle. The Jakarta Post, pp. 1-13. Retrieved from http://www. thejakartapost.com/longform/2016/05/31/the-fitness-phenomenon.html

Arthur, M. W., Hawkins, J. D., Brown, E. C., Briney, J. S., Oesterle, S., \& Abbot, R. D. (2010). Implementation of the Communities That Care prevention system by coalitions in the Community Youth Development Study. Journal of Community Psychology, 28(2), 245-258. https://doi.org/10.1002/jcop.20362

Australia - Indonesia Partnership for Health Systems Strengthening. (2016). Restoring the function of Puskesmas.

Aziza, K. S. (2013, February 27). Dokter Harus Ikhlas Layani Pasien yang Membeludak. Kompas.Com. Retrieved from https://bola.kompas.com/read/2013/02/27/14290931/dokter.harus.ikhlas.layani.pasien.yang. membeludak

Balog, J. E. (2005). The Meaning of Health. American Journal of Health Education, 36(5), 266-271. Retrieved from http://files.eric.ed.gov/fulltext/EJ792826.pdf

Barrera Jr., M., Castro, F. G., Strycker, L. A., \& Toobert, D. J. (2013). Cultural Adaptations of Behavioral Health Interventions: A Progress Report. Journal of Consulting and Clinical Psychology, 81(2), 196-205. https:// doi.org/10.1037/a0027085

Bartholomew Eldredge, L. K., Markham, C. M., Ruiter, R. A. C., Fernandez, M. E., Kok, G., \& Parcel, G. S. (2016). Planning health promotion programs: an Intervention Mapping approach (Fourth). San Francisco, CA, USA: Jossey-Bass. 
Baruth, M., Bopp, M., Webb, B. L., \& Peterson, J. A. (2015). The Role and Influence of Faith Leaders on HealthRelated Issues and Programs in their Congregation. Journal of Religion and Health, 54(5), 1747-1759. https://doi.org/10.1007/s10943-014-9924-1

Basu, A. (2010). Communicating health as an impossibility: Sex work, HIV/AIDS, and the dance of hope and hopelessness. Southern Communication Journal, 75(4), 413-432. https://doi.org/10.1080/104179 4x.2010.504452

Bauermeister, J. A., Pingel, E. S., Sirdenis, T. K., Andrzejewski, J., Gillard, G., \& Harper, G. W. (2017). Ensuring community participation during program planning: Lessons learned during the development of a HIV/STI program for young sexual and gender minorities. American Journal of Community Psychology, 60(1-2), 215-228. https://doi.org/10.1002/ajcp.12147

Berman, P. A. (1984). Village health workers in Java, Indonesia: coverage and equity. Social Science and Medicine, 19(4), 411-422. https://doi.org/10.1016/0277-9536(84)90199-0

Betancourt, J. R., Green, A. R., \& Carrillo, J. E. (2002). Cultural competence in health care: emerging frameworks and practical approaches. The Commonwealth Fund. USA. Retrieved from www.cmwf.org

Betancourt, J. R., Green, A. R., Carrillo, J. E., \& Park, E. R. (2005). Cultural competence and health care disparities: Key perspectives and trends. Health Affairs, 24(2), 499-505. https://doi.org/10.1377/hlthaff.24.2.499

Betsch, C., Böhm, R., Airhihenbuwa, C. O., Butler, R., Chapman, G. B., Haase, N., ... Uskul, A. K. (2016). Improving medical decision making and health promotion through culture-sensitive health communication: An agenda for science and practice. Medical Decision Making, 36(7), 811-833. https://doi. org/10.1177/0272989X15600434

Bosma, L. M., Sieving, R. E., Ericson, A., Russ, P., Cavender, L., \& Bonine, M. (2010). Elements for Successful Collaboration and University Partners: The Lead Peace Partnership. Journal of School Health, 80(10), 501507.

Bradshaw, C., Atkinson, S., \& Doody, O. (2017). Employing a Qualitative Description Approach in Health Care Research. Global Qualitative Nursing Research, 4. https://doi.org/10.1177/2333393617742282

Brown, L. D., Feinberg, M. E., \& Greenberg, M. T. (2010). Determinants of community coalition ability to support evidence-based programs. Prevention Science, 11(3), 287-297. https://doi.org/10.1007/s11121-010-0173-6

Brug, J., Oenema, A., \& Ferreira, I. (2005). Theory, evidence and Intervention Mapping to improve behavior nutrition and physical activity interventions. International Journal of Behavioral Nutrition and Physical Activity, 2(1), 2. https://doi.org/10.1186/1479-5868-2-2

Butterfoss, F. D. (2006). Process Evaluation for Community Participation. Annual Review of Public Health, 27(1), 323-340. https://doi.org/10.1146/annurev.publhealth.27.021405.102207

Butterfoss, F. D., Goodman, R. M., \& Wandersman, A. (1993). Community coalitions for prevention and health promotion. Health Education Research, 8(3), 315-330. https://doi.org/10.1093/her/8.3.315

Butterfoss, F. D., Goodman, R. M., \& Wandersman, A. (1996). Community coalitions for prevention and health promotion: Factors predicting satisfaction, participation, and planning. Health Education and Behavior, 23(1), 65-79. https://doi.org/10.1177/109019819602300105

Butterfoss, F. D., \& Kegler, M. C. (2002). Toward a Comprehensive Understanding of Community Coalitions: Moving from Practice to Theory. In R. J. DiClemente, R. A. Crosby, \& M. C. Kegler (Eds.), Emerging theories in health promotion practice and research : strategies for improving public health (First, pp. 157193). San Francisco, CA: Jossey-Bass. https://doi.org/10.1345/aph.1c251

Butterfoss, F. D., \& Kegler, M. C. (2009). The community coalition action theory. In R. J. DiClemente, R. A Crosby, \& M. C. Kegler (Eds.), Emerging theories in health promotion practice and research (2nd ed., pp. 237-276). San Francisco, CA: Jossey-Bass. Retrieved from http://www.hpforum.org.nz/

Butterfoss, F. D., \& Kegler, M. C. (2012). A coalition model for community action. In M. Minkler (Ed.), Community organizing and community building for health and welfare (pp. 309-328). New Brunswick: Rutgers University Press. Retrieved from htpps://muse.jhu.edu/.

Carey, M. A., \& Asbury, J.-E. (2016). Focus Group Research. Routledge Taylor \& Francis Group. New York: Routledge Taylor \& Francis Group. Retrieved from https://doi.org/10.4324/9781315428376 


\section{References}

Carina, J. (2017, November 4). Banyak Orang Stres di Jakarta, Anies Ingin Ada Psikolog di Puskesmas. Kompas. Com. Retrieved from https://megapolitan.kompas.com/read/2017/11/04/11000061/banyak-orang-stres-dijakarta-anies-ingin-ada-psikolog-di-puskesmas

Caturiningsih, R., Mestuti, H., \& Atik, N. S. (2016). Knowledge relationship with attitude and motivation cadre presence in the service Posyandu in the Village Tumpang Krasak. Journal of Midwifery and Health, 04, 23-33.

Charmaz, K. (2012). The Power and Potential of Grounded Theory. Medical Sociology Online, 6(3), 2-15. Retrieved from http://www.medicalsociologyonline.org/resources/Vol6Iss3/MSo-600x_The-Power-andPotential-Grounded-Theory_Charmaz.pdf

Clarke-McMullen, D. M. (2010). Evaluation of a successful fetal alcohol spectrum disorder coalition in Ontario, Canada. Public Health Nursing, 27(3), 240-247. https://doi.org/10.1111/j.1525-1446.2010.00849.x

Clarke, V., Hayfield, N., Moller, N., \& Tischner, I. (2017). Once Upon A Time. . . : Story Completion Methods. In V. Braun, V. Clarke, \& D. Gray (Eds.), Collecting Qualitative Data: A Practical Guide to Textual, Media and Virtual Techniques (pp. 45-70). Cambridge: Cambridge University Press. Retrieved from http://www. cambridge.org/gb/academic/subjects/psychology/psychology-research-methods-and-statistics/collectingqualitative-d

Conron, H. (2014, February). Jakarta's Garbage Time Bomb. Jakarta Globe. Retrieved from http://jakartaglobe. beritasatu.com/news/jakartas-garbage-time-bomb/

Conti, A. A. (2018). Historical evolution of the concept of health in Western medicine. Acta Bio Medica, 89(3), 352-354. https://doi.org/10.23750/abm.v89i3.6739

Cooper, S., \& Thorogood, N. (2013). Social construction of health and health promotion. In L. Cragg, M. Davies, \& W. Macdowall (Eds.), Health Promotion Theory (Second). Berkshire: Open University Press.

Corbin, J. H., Fernandez, M. E., \& Mullen, P. D. (2015). Evaluation of a Community-Academic Partnership: Lessons From Latinos in a Network for Cancer Control. Health Promotion Practice, 16(3), 345-353. https:// doi.org/10.1177/1524839914558514

Cragg, L., Davies, M., \& Macdowall, W. (Eds.). (2013). Health Promotion Theory. Open University Press (Second). Berkshire: Open University Press.

Cragg, L., Nutland, W., \& Hickson, F. (2015). Planning health promotion interventions. In W. Nutlands \& L. Cragg (Eds.), Health Promotion Practice (Second, pp. 14-30). Berkshire: Open University Press.

Cramer, M. E., Atwood, J. R., \& Stoner, J. A. (2006). A Conceptual Model for Understanding Effective Coalitions Involved in Health Promotion Programing. Public Health Nursing, 23(1), 67-73. https://doi.org/10.1007/97894-009-1005-8_17

Crosby, R. A., Kegler, M. C., \& DiClemente, R. J. (2002). Understanding and applying theory in health promotion practice and research. In R. J. DiClemente, R. A. Crosby, \& M. C. Kegler (Eds.), Emerging theories in health promotion practice and research : strategies for improving public health (First, pp. 1-15). San Francisco, CA: John Wiley \& Sons, Inc. https://doi.org/10.1345/aph.1c251

Cross, R., Davis, S., \& O’Neil, I. (2017). Health communication: Theoretical and critical perspectives. Cambridge, UK: Polity Press. Retrieved from http://web.b.ebscohost.com/ehost/ebookviewer/ebook/bmxlYmtfXzE0OTg 1NTJfX0FO0?sid=b2c5ee8f-a4a7-444d-9a95-67929fa10a66@pdc-v-sessmgr03\&vid=0\&format=EK\&rid=1

Cybriwsky, R., \& Ford, L. R. (2001). City profile Jakarta. Cities, 18(3), 199-210. https://doi.org/10.1016/S02642751(01)00004-X

Cyril, S., Smith, B. J., \& Renzaho, A. M. N. (2016). Systematic review of empowerment measures in health promotion. Health Promotion International, 31(4), 809-826. https://doi.org/10.1093/heapro/dav059

D’Ambruoso, L., Achadi, E., Adisasmita, A., Izati, Y., Makowiecka, K., \& Hussein, J. (2009). Assessing quality of care provided by Indonesian village midwives with a confidential enquiry. Midwifery, 25(5), 528-539. https://doi.org/10.1016/j.midw.2007.08.008

Dalum, P., Schaalma, H., \& Kok, G. (2012). The development of an adolescent smoking cessation interventionan Intervention Mapping approach to planning. Health Education Research, 27(1), 172-181. https://doi. org/10.1093/her/cyr044 
Damarjati, D. (2016, November 23). Penambahan Ruang Terbuka Hijau Jakarta Terancam Gagal Penuhi Target. DetikNews. Retrieved from https://news.detik.com/berita/3352820/penambahan-ruang-terbuka-hijaujakarta-terancam-gagal-penuhi-target

Dewi, S. W. (2015, May 20). Half of Jakarta professionals yet to live healthy: Survey. The Jakarta Post. Retrieved from http://www.thejakartapost.com/news/2015/05/20/half-jakarta-professionals-yet-live-healthy-survey. html

Dewi, S. W. (2016, December 26). Traffic jams move outside Jakarta. The Jakarta Post. Retrieved from http:// www.thejakartapost.com/news/2014/12/26/traffic-jams-move-outside-jakarta.html

Dhorothea. (2016, November 9). Boleh Makan Berlebihan, asal Olahraga. Kompas.Com. Retrieved from http:// health.kompas.com/read/2016/11/09/111600623/boleh.makan.berlebihan.asal.olahraga

Directorate General of Public Administration. (2015). Population Data. Directorate General of Public Administration. Jakarta.

Directorate of Health Promotion and Community Empowerment. (2016). Health promotion. Retrieved September 19, 2019, from http://promkes.kemkes.go.id/promosi-kesehatan

Doll, M., Harper, G. W., Robles-Schrader, G. M., Johnson, J., Bangi, A. K., \& Velagaleti, S. (2012). Perspectives of community partners and researchers about factors impacting coalition functioning over time. Journal of Prevention \& Intervention in the Community, 40(2), 87-102. https://doi.org/10.1080/10852352.2012.660120

Dorimulu, P. (2019). Health Minister Vows to Fast-Track Private Hospital Permits. Retrieved January 16, 2020 , from https://jakartaglobe.id/business/health-minister-vows-to-fasttrack-private-hospital-permits

Duku, S. K. O., Nketiah-Amponsah, E., Janssens, W., \& Pradhan, M. (2018). Perceptions of healthcare quality in Ghana: Does health insurance status matter? PLOS ONE, 13(1), 1-17. https://doi.org/10.1371/journal. pone.0190911

Elo, S., \& Kyngäs, H. (2008). The qualitative content analysis process. Journal of Advanced Nursing, 62(1), 107-115. https://doi.org/10.1111/j.1365-2648.2007.04569.x

Enneman, A. (2014). Legally regulated terms of employment and social security for part-time workers in the Netherlands.

European Patients' Forum. (2017). Patients'Perceptions of Quality in Healthcare. Brussels, Belgium. Retrieved from http://www.eu-patient.eu/globalassets/policy/quality-of-care/quality-survey-report.pdf

Feighery, E., \& Rogers, T. (1990). How-to guides on community health promotion. Guide 12: Buildingand maintainingeffective coalitions. Palo Alto, CA: Stanford Health Promotion Resource Center.

Fertman, C. I., Allensworth, D. D., \& Auld, M. E. (2017). What are health promotion programs? In C. I. Fertman \& D. D. Allensworth (Eds.), Health promotion programs : from theory to practice (Second). San Francisco, CA: Jossey-Bass. Retrieved from http://web.b.ebscohost.com/ehost/ebookviewer/ebook/bmxlYmtfXzEzODQw MzdfX0FO0?sid=07af99b7-a637-4927-b837-537c36520dc4@sessionmgr102\&vid=0\& format=EB\&rid=1

Fijal, D., \& Beagan, B. L. (2019). Indigenous perspectives on health: Integration with a Canadian model of practice. Canadian Journal of Occupational Therapy, XX(X), 1-12. https://doi.org/10.1177/0008417419832284

Finch, H., \& Lewis, J. (2003). Focus group. In J. Ritchie \& J. Lewis (Eds.), Qualitative research practice: A guide for social science students and researchers. London: SAGE Publications.

Flores, J. E., Montgomery, S., \& Lee, J. W. (2005). Organization and staffing barriers to parent involvement in teen pregnancy prevention programs: Challenges for community partnerships. Journal of Adolescent Health, 37(3 SUPPL.), 108-114. https://doi.org/10.1016/j.jadohealth.2005.05.003

Foronda, C. L. (2008). A concept analysis of cultural competence. Journal of Transcultural Nursing, 19(3), 207-212. https://doi.org/10.1177/1043659608317093

Frass, L. R., Hopkins, X. L., Smith, L. U., Kyle, J., VanderKnyff, J., \& Hand, G. A. (2014). A collaborative approach in workforce assessment: South Carolina's strategies for high response rates. Health Promotion Practice, 15(1, Suppl), 14S-22S. https://doi.org/10.1177/1524839913509273

Galea, S., Freudenberg, N., \& Vlahov, D. (2005). Cities and population health. Social Science and Medicine, 60(5), 1017-1033. https://doi.org/10.1016/j.socscimed.2004.06.036 


\section{References}

Gardois, P., Booth, A., Goyder, E., \& Ryan, T. (2014). Health promotion interventions for increasing stroke awareness in ethnic minorities: a systematic review of the literature. BMC Public Health, 14(1), 409. https:// doi.org/10.1186/1471-2458-14-409

Gerassi, L. B., \& Nichols, A. (2018). Heterogeneous perspectives in coalitions and community-based responses to sex trafficking and commercial sexual exploitation: Implications for practice. Journal of Social Service Research, 44(1), 63-77. https://doi.org/10.1080/01488376.2017.1401028

Gilmore, G. (2012). Needs and capacity assessment strategies for health education and health promotion. (4th ed.) Burlington, MA: Jones \& Bartlett Learning. Retrieved from https://books.google.nl/books?hl=en\&lr=\&id=3 W65huBIZcC\&oi=fnd\&pg=PR1\&dq=Gilmore, + G. + D. $+(2012) .+$ Needs + and + Capacity + Assessment + Strat egies + for + Health+Education + and + Health + Promotion: + Jones $+\% 26+$ Bartlett+Learning\&ots $=$ Irc 1 yub5ck\&s ig=-y0gfpe6RtaUKhS5FzyXNXZOs2o\#

Gottwald, M., \& Goodman-Brown, J. (2012). A Guide To Practical Health Promotion. Berkshire: Open University Press. Retrieved from http://web.b.ebscohost.com/ehost/ebookviewer/ebook/bmxlYmtfXzQ4M Tc4OV9fQU41?sid=c4f7e024-c3ed-47d4-b993-b5d9f4b01ede@sessionmgr102\&vid=0\&format=EB\&rid=1

Graham, H., \& White, P. C. L. (2016). Social determinants and lifestyles : integrating environmental and public health perspectives. Public Health, 141, 270-278. https://doi.org/10.1016/j.puhe.2016.09.019

Green, J., Cross, R., Woodall, J., \& Tones, K. (2019). Health Promotion: Planning and Strategies. Sage (4th ed.) London: Sage Publications.

Gregg, J., \& O’Hara, L. (2007). Values and principles evident in current health promotion practice. Health Promotion Journal of Australia, 18, 7-11. https://doi.org/10.1071/HE07007

Hadiwinoto, S., \& Leitmann, J. (1994). Urban Environmental Profile - Jakarta. Cities, 11(3), 153-157. https://doi. org/Doi 10.1016/0264-2751(94)90056-6

Harper, G. W., Contreras, R., Bangi, A., \& Pedraza, A. (2003). Collaborative Process Evaluation: Enhancing Community Relevance and Cultural Appropriateness in HIV Prevention. Journal of Prevention \& Intervention in the Community, 26(2), 53-69. https://doi.org/10.1300/J005v26n02

Harper, G. W., \& Salina, D. D. (2000). Building collaborative partnerships to improve community-based HIV prevention research. Journal of Prevention \& Intervention in the Community, 19(1), 1-20. https://doi. org/10.1300/J005v19n01

Harwell, M. R. (2011). Research Design in Qualitative/Quantitative/ Mixed Methods. In C. F. Conrad \& R. C. Serlin (Eds.), The SAGE Handbook for Research in Education: Pursuing Ideas as the Keystone of Exemplary Inquiry (2nd ed., pp. 147-182). California: SAGE Publications, Inc. https://doi.org/10.4135/9781412961288. $\mathrm{n} 380$

Hassan, S. H. (2015). Effects of Religious Behavior on Health-Related Lifestyles of Muslims in Malaysia. Journal of Religion and Health, 54(4), 1238-1248. https://doi.org/10.1007/s10943-014-9861-z

Hays, J. (2008). Environmental issues and pollution in Indonesia. Retrieved April 26, 2017, from http:// factsanddetails.com/indonesia/Nature_Science_Animals/sub6_8c/entry-4090.html

Hays, K., \& Aranda, M. P. (2016). Faith-Based Mental Health Interventions With African Americans: A Review. Research on Social Work Practice, 26(7), 777-789. https://doi.org/10.1177/1049731515569356

Health PPSDM Agency. (2015). Perencanaan kebutuhan SDM kesehatan berdasarkan standar ketenagaan minimal. Jakarta. Retrieved from https://anzdoc.com/perencanaan-kebutuhan-sdm-kesehatan-berdasarkanstandar-kete.html

Health Services of DKI Jakarta. The Regulation of the Head of Health Services of Special Capital Region of Jakarta number 2131 of 2015, Pub. L. No. 12 /SE/2015 (2015). Indonesia: Health Services of Special Capital Region of Jakarta. Retrieved from https://kepegawaiandinkes.files.wordpress.com/2015/06/draft-peraturannon-pns.pdf

Heesterbeek, H., Anderson, R. M., Andreasen, V., Bansal, S., Angelis, D. De, Dye, C., ... Isaac Newton Institute IDD Collaboration. (2015). Modeling infectious disease dynamics in the complex landscape of global health Science, 347(6227). https://doi.org/10.1126/science.aaa4339 
Herlinda, W. D. (2015, October 17). Rumah Sakit di Jakarta Memerlukan Lebih Banyak Psikolog. Bisnis. Com. Retrieved from http://lifestyle.bisnis.com/read/20151017/106/483201/rs-di-jakarta-butuh-lebih-banyakpsikolog

Hickson, F. (2015). Concepts in health promotion. In W. Nutlands \& L. Cragg (Eds.), Health Promotion Practice (Second, pp. 3-13). Berkshire: Open University Press.

Hidajat, L. L. (2005). Health-sickness perspective From Motivation Value Type and Personality Trends in Javaness Bali community. University of Gadjah Mada. University of Gadjah Mada.

Hsieh, H. F., \& Shannon, S. E. (2005). Three Approaches to Qualitative Content Analysis. Qualitative Health Research, 15(9), 1277-1288. https://doi.org/10.1177/1049732305276687

Huberty, J. L., Balluff, M., O’Dell, M., \& Peterson, K. (2010). From good ideas to actions: A model-driven community collaborative to prevent childhood obesity. Preventive Medicine:, 50(Suppl), S36-S43. https:// doi.org/10.1016/j.ypmed.2009.08.013

Hussein, M. El, Hirst, S., Salyers, V., \& Osuji, J. (2014). Using Grounded Theory as a Method of Inquiry: Advantages and Disadvantages. Qualitative Report, 19(27), 1-15. Retrieved from http://www.nova.edu/ssss/ QR/QR19/el-hussein13.pdf

Ibrahim, S., \& Sidani, S. (2014). Community Based HIV Prevention Intervention in Developing Countries : A Systematic Review. Advances in Nursing, 2014.

Ika. (2015). Minim Psikolog, Ribuan Penderita Gangguan Jiwa Belum Tertangani. Retrieved January 10, 2017, from https://ugm.ac.id/id/berita/9715-minim-psikolog-ribuan-penderita-gangguan-jiwa-belum-tertangani

Isaak, C. A., \& Marchessault, G. (2008). Meaning of Health: The Perspectives of Aboriginal Adults and Youth in a Northern Manitoba First Nations Community. Canadian Journal of Diabetes, 32(2), 114-122. https://doi. org/10.1016/S1499-2671(08)22008-3

Ismail, S. (2015, November 26). Jakarta taking measures to mitigate impact of floods. Channel News Asia. Retrieved from http://www.channelnewsasia.com/news/asiapacific/jakarta-taking-measures/2294810.html

Jack, L., Grim, M., \& Auld, M. E. (2012). Health Promotion Practice Expands Focus on Global Health Promotion. Health Promotion Practice, 13(3), 289-292. https://doi.org/10.1177/1524839912443244

Jackson, A. C., \& Segal, S. P. (Eds.). (2014). Social work health and mental health: Practice, research and programs (second). New York: Routledge. Retrieved from https://www.ncbi.nlm.nih.gov/ pubmed/12365748? otool=inlmaulib

Jagosh, J., Bush, P. L., Salsberg, J., Macaulay, A. C., Greenhalgh, T., Wong, G., ... Pluye, P. (2015). A realist evaluation of community-based participatory research: Partnership synergy, trust building and related ripple effects. BMC Public Health, 15(1), 1-11. https://doi.org/10.1186/s12889-015-1949-1

Jakarta Provincial Health Agencies. (2016). Health Profile of DKI Jakarta Province 2016. Jakarta. Retrieved from http:/dinkes.jakarta.go.id/berita/profil-kesehatan-provinsi-dki-jakarta-tahun-2016/

Jayanthi, T. P., Suresh, S., \& Padmanaban, P. (2015). Primary health centres: Preferred option for birthing care in Tamilnadu, India, from users' perspectives. Journal of Health, Population and Nutrition, 33(1), 177-186.

John, J., \& Miller, A. R. (2015). Health care service quality: the importance of customer perceptions. In Bahn K. (Ed.), Developments in Marketing Science: Proceedings of the Academy of Marketing Science (Proceeding, pp. 373-374). Switzerland: Springer, Cham. https://doi.org/https://doi.org/10.1007/978-3-319-17046-6_76

Jong, H. N. (2015, October 9). Indonesia in state of waste emergency. The Jakarta Post. Retrieved from http:// www.thejakartapost.com/news/2015/10/09/indonesia-state-waste-emergency.html

Jongen, C. S., McCalman, J., \& Bainbridge, R. G. (2017). The implementation and evaluation of health promotion services and programs to improve cultural competency: A systematic scoping review. Frontiers in Public Health, 5(February). https://doi.org/10.3389/fpubh.2017.00024

Jongepier, E. (2017). Illness and Dutch employment law. Retrieved January 12, 2017, from https://www. blenheim.nl/employment-law-netherlands/illness-employment

Jormfeldt, H., Svedberg, P., Fridlund, B., \& Arvidsson, B. (2007). Perceptions of the concept of health among nurses working in mental health services: A phenomenographic study. International Journal of Mental Health Nursing, 16(1), 50-56. https://doi.org/10.1111/j.1447-0349.2006.00444.x 


\section{References}

Kahissay, M. H., Fenta, T. G., \& Boon, H. (2017). Beliefs and perception of ill-health causation: a socio-cultural qualitative study in rural North-Eastern Ethiopia. BMC Public Health, 17(1), 124. https://doi.org/10.1186/ s12889-017-4052-y

Karyana, M., Devine, A., Kenangalem, E., Burdarm, L., Poespoprodjo, J. R., Vemuri, R., ... Yeung, S. (2016). Treatment-seeking behaviour and associated costs for malaria in Papua, Indonesia. Malaria Journal, 15(536), 1-12. https://doi.org/10.1186/s12936-016-1588-8

Kayral, I. H. (2014). Perceived Service Quality in Healthcare Organizations and a Research in Ankara by Hospital Type. Journal of Ankara Studies, 2(21), 22-22. Retrieved from http://www.journalagent.com/jas/ pdfs/JAS_2_1_22_34.pdf

Kegler, M. C., Hall, S. M., \& Kiser, M. (2010). Facilitators, challenges, and collaborative activities in faith and health partnerships to address health disparities. Health Education \& Behavior, 37(5), 665-679. https://doi. org/10.1177/1090198110363882

Keleher, H., \& Armstrong, R. (2005). Evidence-based mental health promotion resource. Melbourne, Victoria: Public Health Group Victorian Government Department of Human Services.

Khamis, K., \& Njau, B. (2016). Health care worker's perception about the quality of health care at the outpatient department in Mwananyamala Hospital in Dar es Salaam, Tanzania. Tanzania Journal of Health Research, 18(1), 1-9. https://doi.org/10.4314/THRB.V18I1.

Kok, G., Gottlieb, N. H., Peters, G. J. Y., Mullen, P. D., Parcel, G. S., Ruiter, R. A. C., .. Bartholomew, L. K. (2016). A taxonomy of behaviour change methods: an Intervention Mapping approach. Health Psychology Review, 10(3), 297-312. https://doi.org/10.1080/17437199.2015.1077155

Krefis, A., Augustin, M., Schlünzen, K., Oßenbrügge, J., \& Augustin, J. (2018). How Does the Urban Environment Affect Health and Well-Being? A Systematic Review. Urban Science, 2(1), 1-21. https://doi.org/10.3390/ urbansci2010021

Kreuter, M. W., Lezin, N. A., \& Young, L. A. (2010). Health Promotion Practice Evaluating Collaborative Mechanisms : Implications for Practitioners, 1(1), 49-63. https://doi.org/10.1177/152483990000100109

Kreuter, M. W., Lukwago, S. N., Bucholtz, R. D., Clark, E. M., \& Sanders-Thompson, V. (2003). Achieving cultural appropriateness in health promotion programs: targeted and tailored approaches. Health Education and Behavior, 30(2), 133-146. https://doi.org/10.1177/1090198102251021

Kreuter, M. W., \& McClure, S. M. (2004). The Role of Culture in Health Communication. Annual Review of Public Health, 25(1), 439-455. https://doi.org/10.1146/annurev.publhealth.25.101802.123000

Kruger, D. J., Lewis, Y., \& Schlemmer, E. (2010). Mapping a Message for Faith Leaders: Encouraging Community Health Promotion With Local Health Data. Health Promotion Practice, 11(6), 837-844. https:// doi.org/10.1177/1524839908328995

Kwak, L., Kremers, S. P. J., Werkman, A., Visscher, T. L. S., Van Baak, M. A., \& Brug, J. (2007). The NHFNRG In Balance-project: The application of Intervention Mapping in the development, implementation and evaluation of weight gain prevention at the worksite. Obesity Reviews, 8(4), 347-361. https://doi.org/10.1111/ j.1467-789X.2006.00304.x

Laverack, G. (2007). Health promotion practice: building empowered communities. Berkshire: Open University Press. Retrieved from http://journals.sagepub.com/doi/10.1177/152483990200300315

Laverack, G. (2014). The pocket guide to health promotion. New York: Open University Press. https://doi. org/10.3390/challe8020025

Lembur, J. (2016, July). Menkes Buka 14 Nama RS Penerima Vaksin Palsu, Ini Daftarnya. DetikNews. Retrieved from https://news.detik.com/berita/3253345/menkes-buka-14-nama-rs-penerima-vaksin-palsu-ini-daftarnya

Leung, L. (2015). Validity, reliability, and generalizability in qualitative research. Journal of Family Medicine and Primary Care, 4(3), 324-327. https://doi.org/10.4103/2249-4863.161306

Levesque, A., Li, H., \& Bohémier, M. (2013). Cultural variations in health conceptions: A qualitative approach. Pimatisiwin: A Journal of Aboriginal and Indigenous Community Health, 11(2), 215-229.

Levine, J. M., \& Moreland, R. L. (1998). Small groups. In D. Gilbert, T. Fiske, \& G. Lindzey (Eds.), Handbook of social psychology (4th ed., Vol. 2, pp. 415-469). Boston: McGraw-Hill. 
Liem, A., \& Yuniarti, K. W. (2012). Healthy is ... "An Indigenous Psychological Analysis of Teen in Prambanan, Yogyakarta. In National Scientific Meeting UKRIDA. Jakarta: National Scientific Meeting UKRIDA.

Lloyd, J. J., Logan, S., Greaves, C., \& Wyatt, K. (2011). Evidence, theory and context - using intervention mapping to develop a school-based intervention to prevent obesity in children. International Journal of Behavioral Nutrition and Physical Activity, 8(73), 1-15. https://doi.org/10.1186/1479-5868-8-73

Lucas, K., \& Lloyd, B. (2005). Health promotion: evidence and experience. London: SAGE Publications. https:// doi.org/10.1016/B978-0-7020-5401-3.00036-9

MacDonald, T. H. (1998). Rethinking health promotion: a global approach. London: Routledge. Retrieved from http://web.b.ebscohost.com/ehost/ebookviewer/ebook/bmxlYmtfXzQ2MDM0NF9fQU41?sid=51d3df447e28-41ee-a65b-d99f1ac5e66c@sessionmgr103\&vid=1\&format=EB\&rid=1

Mahendradhata, Y., Trisnantoro, L., Listyadewi, S., Soewondo, P., Marthias, T., Harimurti, P., \& Prawira, J. (2017). The Republic of Indonesia health system review. (K. Hort \& W. Patcharanarumol, Eds.), Health Systems in Transition (Vol. 7). India: World Health Organization, Regional Office for South-East Asia.

Marthoenis, M., Aichberger, M. C., \& Schouler-Ocak, M. (2016). Patterns and Determinants of Treatment Seeking among Previously Untreated Psychotic Patients in Aceh Province, Indonesia: A Qualitative Study. Scientifica, 2016, 1-7. https://doi.org/10.1155/2016/9136079

Martono, J. (2011, June 13). Layanan Konsultasi Psikologi di Puskesmas Kota Yogyakarta. Kompasiana. Retrieved from http:/www.kompasiana.com/jk.martono/layanan-konsultasi-psikologi-di-puskesmas-kotayogyakarta_5500e460813311fb16fa8003

Mayring, P. (2014). Qualitative content analysis: theoretical foundation, basic procedures and software solution Social Science Open Access Repository. Klagenfurt: Social Science Open Access Repository. https://doi. org/10.1016/S1479-3709(07)11003-7

Mays, D., Peshkin, B. N., Sharff, M. E., Walker, L. R., Abraham, A. A., Hawkins, K., \& Tercyak, K. P. (2012). Correlates of Adherence to a Telephone-Based Multiple Health Behavior Change Cancer Preventive Intervention for Teens: The Healthy for Life Program (HELP). Health Education \& Behavior, 39(1), 18-26. https://doi.org/10.1177/1090198111404554.Correlates

McCarthy, P. (2003). Urban slums reports: the case of Jakarta, Indonesia. Global Report on Human Settlements 2003, The Challenge of Slums. Jakarta. Retrieved from https://www.un.org/ruleoflaw/files/Challenge of Slums.pdf

McKay, A., \& Wangchuk, D. (2018). Traditional medicine in Bhutan. Asian Medicine, 1(1), 201-218. Retrieved from https://doi.org/10.1163/157342105777996737

Mckinney, M., Fitzgerald, H. E., Winn, D., \& Babcock, P. (2017). Public policy, child development research and boys at risk: Challenging, enduring and necessary partnership. Infant Mental Health Journal, 38(1), 166-176. https://doi.org/10.1002/imhj.21623

McLeroy, K. R., Norton, B. L., Kegler, M. C., Burdine, J. N., \& Sumaya, C. V. (2003). Community-based interventions. American Journal of Public Health, 93(4), 529-533. https://doi.org/10.2105/AJPH.93.4.529

McNeish, R., Rigg, K. K., Tran, Q., \& Hodges, S. (2019). Community-based behavioral health interventions: Developing strong community partnerships. Evaluation and Program Planning, 73(November 2018), 111115. https://doi.org/10.1016/j.evalprogplan.2018.12.005

McPake, B., Edoka, I., Witter, S., Kielmann, K., Taegtmeyer, M., Dieleman, M., ... Cometto, G. (2015). Costeffectiveness of community-based practitioner programmes in Ethiopia, Indonesia and Kenya. Bulletin of the World Health Organization, 93(August 2014), 631-639A. https://doi.org/10.2471/BLT.14.144899

Megawangi, S. R. (2017). Peran dokter layanan primer, menyehatkan penduduk Indonesia. Retrieved September 20, 2019, from http://fk.ugm.ac.id/peran-dokter-layanan-primer-menyehatkan-penduduk-indonesia/

Mendelson, C. (2002). Health Perceptions of Mexican American Women. Journal of Transcultural Nursing, 13(3), 210-217. https://doi.org/10.1177/10459602013003010

Mendoza, R. L. (2009). Is It Really Medicine? The Traditional and Alternative Medicine Act and Informal Health Economy in the Philippines. Asia-Pacific Journal of Public Health, 21(3), 333-345. 


\section{References}

Miller, R. L., Reed, S. J., Chiaramonte, D., Strzyzykowski, T., Spring, H., Acevedo-Polakovich, I. D., ... Ellen, J. M. (2017). Structural and community change outcomes of the Connect-to-Protect coalitions: Trials and triumphs securing adolescent access to HIV prevention, testing, and medical care. American Journal of Community Psychology, 60(1-2), 199-214. https://doi.org/10.1002/ajcp.12162

Ministerie van Sociale Zaken en Werkgelegenheid. (2011). Living and working in the Netherlands: Information for European workers in the Netherlands. Netherlands.

Ministry of Health of the Republic of Indonesia. (2015a). Data and information on 2014: Indonesia health profile. Ministry of Health of the Republic of Indonesia. Jakarta.

Ministry of Health of the Republic of Indonesia. (2015b). The number of primary health service centers Per June 2015. Jakarta.

Ministry of Health of the Republic of Indonesia. The strategic plan of the Ministry of Health in 2015-2019, Pub. L. No. HK.02.02/MENKES/52/2015 (2015). Jakarta, Indonesia: Ministry of Health of the Republic of Indonesia. Retrieved from http://www.depkes.go.id/article/view/15011700003/demam-berdarah-biasanyamulai-meningkat-di-januari.html

Ministry of Health Republic of Indonesia. Health Ministry Decree Number 128 of 2004, Pub. L. No. Number 128 of 2004, Ministry of Health of the Republic of Indonesia (2004). Indonesia: Ministry of Health of the Republic of Indonesia. https://doi.org/10.1016/0021-9924(94)90039-6

Ministry of Health Republic of Indonesia. Minister of Health decree number 585/Menkes/SK/V/2007, Pub. L. No. 585/Menkes/SK/V/2007 (2007). The Republic of Indonesia: Ministry of Health Republic of Indonesia.

Ministry of Health Republic of Indonesia. (2011). Promosi kesehatan di daerah bermasalah kesehatan: panduan bagi petugas kesehatan di Puskesmas. Kementrian Kesehatan Republik Indonesia. Jakarta: Kementrian Kesehatan Republik Indonesia.

Ministry of Health Republic of Indonesia. (2013). National Health Survey 2013. Jakarta. https://doi.org/10.1007/ s13398-014-0173-7.2

Ministry of Health Republic of Indonesia. Health Ministry Regulation Number 75 of 2014, Pub. L. No. Number 75 of 2014 (2014). The Republic of Indonesia: Ministry of Health Republic of Indonesia.

Ministry of Health Republic of Indonesia. The Regulation of the Health Minister of the Republic of Indonesia Number 59 of 2014, Pub. L. No. Number 59 of 2014, Health Ministry of the Republic of Indonesia (2014). Republic of Indonesia: Health Ministry of the Republic of Indonesia. Retrieved from http://www.depkes. go.id/resources/download/peraturan/pmk-59-thn-2014-ttg-standar-tarif-jkn.pdf

Ministry of Health Republic of Indonesia. The Regulation of the Health Minister of the Republic of Indonesia Number 46 of 2015, Pub. L. No. Number 46 of 2015, Health Ministry of the Republic of Indonesia (2015) Republic of Indonesia: Health Ministry of the Republic of Indonesia. https://doi.org/10.1073/pnas.0703993104

Ministry of Health Republic of Indonesia. (2016). Indonesia's Health Profile 2015. (D. Budijanto, Yudianto, B. Hardhana, \& T. A. Soenardi, Eds.) (2015th ed.). Jakarta: Kementerian Kesehatan Republik Indonesia. https:// doi.org/351.077 Ind

Ministry of Health Republic of Indonesia. (2017a). Performance Report of 2016 - Ministry of Health Republic of Indonesia. Ministry of Health Republic of Indonesia. Jakarta.

Ministry of Health Republic of Indonesia. (2017b). Puskemas Ujung Tombak Kesehatan Masyarakat.

Ministry of Health Republic of Indonesia. Regulation of Ministry of Health Republic of Indonesia number HK.01.07/MENKES/187/2017, Pub. L. No. HK.01.07/MENKES/187/2017, 1 (2017). Republic of Indonesia: Ministry of Health Republic of Indonesia.

Ministry of Health Republic of Indonesia. (2018a). 4 Target Kesehatan ini Harus Tercapai di 2019.

Ministry of Health Republic of Indonesia. (2018b). Performance Report of 2017 - Ministry of Health Republic of Indonesia. Jakarta.

Ministry of Health Republic of Indonesia. The Regulation of the Health Minister of the Republic of Indonesia Number 44 of 2018, Pub. L. No. Number 44 of 2018 (2018). the Republic of Indonesia: Ministry of Health Republic of Indonesia. https://doi.org/10.1093/imamci/dnt037 
Ministry of Health Republic of Indonesia. Health Ministry Regulation Number 3 of 2019, Pub. L. No. Number 3 of 2019 (2019). The Republic of Indonesia: Ministry of Health Republic of Indonesia. https://doi. org/.1037//0033-2909.I26.1.78

Mirzaei-alavijeh, M., Karami-matin, B., Van Lieshout, S., Mahboubi, M., \& Zinat-Motlagh, F. (2016). Appling intervention mapping approach to promoting healthy diet intention among diabetic patients: an efficacy study. Arvand Journal of Health \& Medical Sciences, 1(4), 243-250.

Mitropoulos, P., Vasileiou, K., \& Mitropoulos, I. (2018). Understanding quality and satisfaction in public hospital services: A nationwide inpatient survey in Greece. Journal of Retailing and Consumer Services, 40, $270-275$. https://doi.org/10.1016/j.jretconser.2017.03.004

Mizrahi, T., \& Rosenthal, B. B. (2001). Complexities of Coalition Building: Leaders' Successes, Strategies, Struggles, and Solutions. Social Work, 46(1), 63-78. https://doi.org/10.1093/sw/46.1.63

Motley, M., Holmes, A., Hill, J., Plumb, K., \& Zoellner, J. (2013). Evaluating community capacity to address obesity in the Dan River Region: A case study. American Journal of Health Behavior, 37(2), 208-217. https:// doi.org/10.5993/AJHB.37.2.8

Nazri, C., Yamazaki, C., Kameo, S., Herawati, D. M. D., Sekarwana, N., Raksanagara, A., \& Koyama, H. (2016). Factors influencing mother's participation in Posyandu for improving nutritional status of children under-five in Aceh Utara district, Aceh province, Indonesia. BMC Public Health, 16(1), 1-9. https://doi.org/10.1186/ s12889-016-2732-7

Netto, G., Bhopal, R., Lederle, N., Khatoon, J., \& Jackson, A. (2010). How can health promotion interventions be adapted for minority ethnic communities? Five principles for guiding the development of behavioural interventions. Health Promotion International, 25(2), 248-257. https://doi.org/10.1093/heapro/daq012

Noar, S. M., Chabot, M., \& Zimmerman, R. S. (2008). Applying health behavior theory to multiple behavior change: Considerations and approaches. Preventive Medicine, 46(3), 275-280. https://doi.org/10.1016/j. ypmed.2007.08.001

Noble, H., \& Smith, J. (2015). Issues of validity and reliability in qualitative research. Evidence-Based Nursing, 18(2), 34-35. https://doi.org/10.1136/eb-2015-102054

Nurhayati, \& Widowati, L. (2017). The use of traditional health care among Indonesian Family. Health Science Journal of Indonesia, 8(1), 30-35. https://doi.org/10.22435/hsji.v8i1.5600.

Nutlands, W., \& Cragg, L. (Eds.). (2015). Health Promotion Practice (Second). Berkshire: Open University Press.

Pasang, H., Moore, G. A., \& Sitorus, G. (2007). Neighbourhood-based waste management: A solution for solid waste problems in Jakarta, Indonesia. Waste Management, 27(12), 1924-1938. https://doi.org/10.1016/j. wasman.2006.09.010

Patel, V., Minas, I. H., Cohen, A., \& Prince, M. J. (Eds.). (2014). Global mental health: Principles and practice. New York: Oxford University Press. Retrieved from https://oxfordmedicine.com/view/10.1093/ med/9780199920181.001.0001/med-9780199920181

Peltzer, K., \& Machleidt, W. (1992). A traditional (African) approach towards the therapy of schizophrenia and its comparison with western models. Therapeutic Communities, 13(4), 229-242. https://doi.org/10.1037/00219010.69.1.85

Pemunta, N., \& Obara, T. (2012). Toward a Reconceptualization of the "Urban" and "Rural" as Conceptual and Analytical Categories in the Toward a Reconceptualization of the "Urban" and "Rural" as Conceptual and Analytical Categories in the Social Sciences. Arts and Social Sciences Journal, 2012, 1-14. Retrieved from http://astonjournals.com/assj

Pengpid, S., \& Peltzer, K. (2018). Utilization of traditional and complementary medicine in Indonesia: Results of a national survey in 2014-15. Complementary Therapies in Clinical Practice, 33, 156-163. https://doi. org/10.1016/j.ctcp.2018.10.006

Pérez-Rodrigo, C., Wind, M., Hildonen, C., Bjelland, M., Aranceta, J., Klepp, K. I., \& Brug, J. (2005). The pro children intervention: Applying the intervention mapping protocol to develop a school-based fruit and vegetable promotion programme. Annals of Nutrition and Metabolism, 49(4), 267-277. https://doi. org/10.1159/000087249 


\section{References}

Peterson, N. A., Powell, K. G., Treitler, P., Litterer, D., Borys, S., \& Hallcom, D. (2019). The strategic prevention framework in community-based coalitions: Internal processes and associated changes in policies affecting adolescent substance abuse. Children and Youth Services Review, 101, 352-362. https://doi.org/10.1016/j. childyouth.2019.04.004

Phillips, K. L., Chiriboga, D. A., \& Jang, Y. (2012). Satisfaction with care: The role of patient-provider racial/ ethnic concordance and interpersonal sensitivity. Journal of Aging and Health, 24(7), 1079-1090. https://doi. org/10.1177/0898264312453068

Pickett, K. E., \& Wilkinson, R. G. (2015). Income inequality and health : A causal review. Social Science \& Medicine, 128, 316-326. https://doi.org/10.1016/j.socscimed.2014.12.031

Poland, B., Krupa, G., \& Mccall, D. (2009). Settings for Health Promotion: An Analytic Framework to Guide Intervention Design and Implementation. Health Promotion Practice, 10(4), 505-516. https://doi. org/10.1177/1524839909341025

Pradesha, N. T. (2015, May 19). Hasil Survei: Warga Jakarta Kebanyakan Kerja, Kurang Olahraga. CNN Indonesia. Retrieved from http://www.cnnindonesia.com/gaya-hidup/20150519174526-255-54296/hasil-survei-wargajakarta-kebanyakan-kerja-kurang-olahraga/

Pramanik, A. (2016). Patients' Perception of Service Quality of Health Care Services in India. Journal of Health Management, 18(2), 205-217. https://doi.org/10.1177/0972063416637695

Pramudiarja, A. U. (2014, October 24). Butuh Curhat Sekalian Cek Kesehatan Mental? Gratis, Datang Saja ke Sini. DetikHealth.

Prathivi, N. (2015, January 11). Enjoying a stress-free life - National. The Jakarta Post. Retrieved from http:// www.thejakartapost.com/news/2015/01/11/yoga-big-city-methods-every-taste.html

Punamäki, R.-L., \& Aschan, H. (1994). Self-care and mastery among primary health care patients. Social Science \& Medicine, 39(5), 733-741. https://doi.org/10.1016/0277-9536(94)90028-0

Putri, T. A. (2013, February 27). Dokter Puskesmas di Jakarta Kelebihan Pasien. Tempo. Retrieved from https:// metro.tempo.co/read/news/2013/02/27/083464134/dokter-puskesmas-di-jakarta-kelebihan-pasien

Qiu, J. (2007). Traditional medicine: a culture in the balance. Nature (Vol. 448). Beijing. https://doi. org/10.1038/448126a

Qusna, D. N. (2016). Pelatihan kader kesehatan. Retrieved September 20, 2019, from https://pkmseyegan. slemankab.go.id/pelatihan-kader-kesehatan/

Raingruber, B. (2014). Health Education, Health Promotion, and Health : What Do These Definitions Have to Do with Nursing? In Contemporary Health Promotion in Nursing Practice (pp. 1-24). Burlington, MA: Jones \& Bartlett Learning. Retrieved from https://books.google.com.au/books?id=GeGq5tJlVHYC

Raza, W. A., Van De Poel, E., Panda, P., Dror, D., \& Bedi, A. (2016). Healthcare seeking behaviour among selfhelp group households in Rural Bihar and Uttar Pradesh, India. BMC Health Services Research, 16(1), 1-13. https://doi.org/10.1186/s12913-015-1254-9

Redding, C. a, Rossi, J. S., Rossi, S. R., Velicer, W. F., Prochaska, J. O., ParticipACTION, ... Wang, J. (2008). Evidence, Theory and Context: Using intervention mapping to develope a worksite physical activity intervention. Translational Behavioral Medicine, 3(1), 56. https://doi.org/10.1007/s13142-011-0021-7

Reed, S. J., Miller, R. L., \& Francisco, V. T. (2014). The influence of community context on how coalitions achieve HIV-preventive structural change. Health Education \& Behavior, 41(1), 100-107. https://doi. org/10.1177/1090198113492766

Reid, K. S., Sekhobo, J. P., Gantner, L. A., Holbrook, M. E. K., Allsopp, M., Whalen, L. B., \& Koren-Roth, A. (2018). A mixed-method evaluation of the New York State Eat Well Play Hard Community Projects: Building local capacity for sustainable childhood obesity prevention. Evaluation and Program Planning, 67(December 2017), 79-88. https://doi.org/10.1016/j.evalprogplan.2017.12.004

Remenapp, A., Broome, B., Maetozo, G., \& Hausenblas, H. (2017). Efficacy of a Multiple Health Behavior Change Intervention on Women's Health Outcomes. Women's Health - Open Journal, 3(1), 8-14. https://doi. org/10.17140/WHOJ-3-116 
Resnicow, K., Baranowski, T., Ahluwalia, J. S., \& Braithwaite, R. L. (1999). Cultural sensitivity in public health: Defined and demystified. Ethnicity and Disease, 9(1), 10-21.

Resnicow, K., Soler, R., Braithwaite, R. L., Ahluwalia, J. S., \& Butler, J. (2000). Cultural sensitivity in substance use prevention. Journal of Community Psychology, 28(3), 271-290. https://doi.org/10.1002/(SICI)15206629(200005)28:3<271::AID-JCOP4>3.0.CO;2-I

Rheenen, G. Van. (1998). Animistic and Western Perspectives of Illness and Healing. International Journal of Frontier Missions, 15(2). Retrieved from http://ijfm.org/PDFs_IJFM/15_2_PDFs/ijfm_15_2.pdf\#page=25

Richter, M. (2003). Traditional Medicines and Traditional Healers in South Africa. Treatment Action Campaign and AIDS Law Project. South Africa.

Rivera-Hernandez, M. (2015). The Role of Religious Leaders in Health Promotion for Older Mexicans with Diabetes. Journal of Religion and Health, 54(1), 303-315. https://doi.org/10.1007/s10943-014-9829-Z

Robertson-James, C., Sawyer, L., Núñez, A., Campoli, B., Robertson, D., DeVilliers, A., ... Alexander, S. (2017). Promoting policy development through community participatory approaches to health promotion: The Philadelphia Ujima experience. Women's Health Issues, 27(Suppl 1), S29-S37. https://doi.org/10.1016/j. whi.2017.09.001

Robinson, K. L., Driedger, M. S., Elliott, S. J., \& Eyles, J. (2006). Understanding Facilitators of and Barriers to Health Promotion Practice. Health Promotion Practice, 7(4), 467-476. https://doi.org/10.1177/1524839905278955

Robinson, N. (2006). Integrated traditional Chinese medicine. Complementary Therapies in Clinical Practice, 12 , 132-140. Retrieved from https://ac.els-cdn.com/S1744388106000089/1-s2.0-S1744388106000089-main.pdf? tid=f3937931-e1e7-4a95-9a62-65dbdfcbd3b9\&acdnat=1543415647_d3eb76359584ddc41074f4ed56451627

Rowling, L., \& Weist, M. (2004). Promoting the Growth, Improvement and Sustainability of School Mental Health Programs Worldwide. International Journal of Mental Health Promotion, 6(2), 3-11. https://doi.org/10.1080/1 4623730.2004.9721925

Rydin, Y., Bleahu, A., Davies, M., Davila, J. D., Friel, S., di Grandis, G., ... Wilson, J. (2012). Shaping Cities for Health: the complexities of planning urban environments in the 21 st century. The Lancet, 379 (Special Issue), 2079-2108. https://doi.org/10.1016/S0140-6736(12)60435-8.Shaping

Sadilek, A., Kautz, H., \& Silenzio, V. (2012). Modeling Spread of Disease from Social Interactions. In Proceedings of the Sixth International AAAI Conference on Weblogs and Social Media (pp. 322-329). Dublin: The AAAI Press.

Sakakibara, B. M., Lear, S. A., Barr, S. I., Benavente, O., Goldsmith, C. H., Silverberg, N. D., ... Eng, J. J. (2017) Development of a chronic disease management program for stroke survivors using intervention mapping: The Stroke Coach. Archives of Physical Medicine and Rehabilitation, O(0), 25. https://doi.org/10.1016/j. apmr.2017.01.019

Samosir, H. A. (2015, May 21). Polusi Udara Sebabkan Hampir 60 Persen Penyakit di Jakarta. CNN Indonesia. Retrieved from https://www.google.nl/search?q=Polusi+Udara+Sebabkan+Hampir+60+Persen+Penyakit+di+Jakarta\&oq=Polusi+Udara+Sebabkan+Hampir+60+Persen+Penyakit+di+Jakarta\&aqs=chrome..69i$57.14310 \mathrm{j} 0 \mathrm{j} 1 \&$ sourceid $=$ chrome $\& \mathrm{ie}=\mathrm{UTF}-8$

Sandelowski, M. (2010). What's in a name? Qualitative description revisited. Research in Nursing and Health, 33(1), 77-84. https://doi.org/10.1002/nur.20362

Sari, E. P. (2016). Upaya Pemberdayaan Komunitas Melalui Sinergi Kader Posyandu Dan Psikolog Puskesmas. Jurnal Ilmiah Psikologi Terapan, 04(01), 53-61.

Saunders, B., Sim, J., Kingstone, T., Baker, S., Waterfield, J., Bartlam, B., ... Jinks, C. (2018). Saturation in qualitative research: exploring its conceptualization and operationalization. Quality and Quantity, 52(4), 1893 1907. https://doi.org/10.1007/s11135-017-0574-8

Schwingel, A., \& Gálvez, P. (2016). Divine interventions: Faith-Based approaches to health promotion programs for Latinos. Journal of Religion and Health, 55(6), 1891-1906. https://doi.org/10.1007/s10943-015-0156-9

Seitanidi, M. M., \& Ryan, A. (2007). A critical review of forms of corporate community involvement: From philanthropy to partnerships. International Journal of Nonprofit and Voluntary Sector Marketing, 12, $247-$ 266. https://doi.org/10.1002/nvsm 


\section{References}

Senitan, M., Alhaiti, A. H., \& Gillespie, J. (2018). Patient satisfaction and experience of primary care in Saudi Arabia: A systematic review. International Journal for Quality in Health Care, 30(10), 751-759. https://doi. org/10.1093/intqhe/mzy104

Setiyani, R. Y. (2018). Layanan psikolog di Puskesmas Yogyakarta, solusi deteksi gangguan jiwa di level bawah. Retrieved November 26, 2018, from https://theconversation.com/amp/layanan-psikolog-di-puskesmasyogyakarta-solusi-deteksi-gangguan-jiwa-di-level-bawah-96484

Setiyawati, D., Blashki, G., Wraith, R., Colucci, E., \& Minas, H. (2014). Indonesian experts' perspectives on a curriculum for psychologists working in primary health care in Indonesia. Health Psychology and Behavioral Medicine, 2(1), 623-639. https://doi.org/10.1080/21642850.2014.951937

Shirazi, M., Shirazi, A., \& Bloom, J. (2015). Developing a Culturally Competent Faith-Based Framework to Promote Breast Cancer Screening Among Afghan Immigrant Women. Journal of Religion and Health, 54(1), 153-159. https://doi.org/10.1007/s10943-013-9793-Z

Shortell, S. M., Zukoski, A. P., Alexander, J. A., Bazzoli, G. J., Conrad, D. A., Hasnain-Wynia, R., ... Margolin, F. S. (2002). Evaluating partnerships for community health improvement: Tracking the footprints. Journal of Health Politics, Policy and Law, 27(1), 49-91. https://doi.org/10.1215/03616878-27-1-49

Simmons, R., Bennett, E., Schwartz, M. L., Sharify, D. T., \& Short, E. (2002). Health education and cultural diversity in the health care setting: tips for the practitioner. Health Promotion Practice, 3(1), 8-11.

Simone, A. (2014). Jakarta: Drawing the City Near. Minneapolis: University of Minnesota Press. https://doi. org/10.1177/009430611562152611

Simone, A. (2015). The urban poor and their ambiv alent exceptionalities. Current Anthropology, 56(S11), S15S23. https://doi.org/10.1086/682283

Singelis, T. M., Garcia, R. I., Barker, J. C., \& Davis, R. E. (2018). An Experimental Test of the Two-Dimensional Theory of Cultural Sensitivity in Health Communication. Journal of Health Communication, 23(4), 321-328. https://doi.org/10.1080/10810730.2018.1443526.An

Smith, B. J., Tang, K. C., \& Nutbeam, D. (2006). WHO health promotion glossary: New terms. Health Promotion International, 21(4), 340-345. https://doi.org/10.1093/heapro/dal033

Snyder, L. B., \& Hamilton, M. A. (2002). A Meta-Analysis of U.S. Health Campaign Effects on Behavior: Emphasize Enforcement, Exposure, and New Information, and Beware the Secular Trend. In R. Hornik (Ed.), Public health communication: evidence for behavior change. New Jersey: Lawrence Erlbaum Associates, Inc. https://doi.org/10.1017/CBO9781107415324.004

Soejoeti, S. Z. (2008). Healthy, pain and disease concepts in socio-cultural context. Cermin Dunia Kedokteran, 3(149), 49-53.

Sofaer, S., \& Firminger, K. (2005). Patient Perceptions of the Quality of Health Services. Annual Review of Public Health, 26(1), 513-559. https://doi.org/10.1146/annurev.publhealth.25.050503.153958

Sokang, Y. A., Westmaas, A. H., \& Kok, G. (2019a). From Physical to Spiritual: A Qualitative Study of Jakartans Health \& Sickness. International Journal of Environmental Research and Public Health, 16(19), 3564. https://doi.org/10.3390/ijerph16193564

Sokang, Y. A., Westmaas, A. H., \& Kok, G. (2019b). Health workers ' reflection on health promotion. Manuscript Submitted for Publication, 1-8.

Sokang, Y. A., Westmaas, A. H., \& Kok, G. (2019c). Jakartans' Perceptions of Health Care Services. Frontiers in Public Health, 7(September), 1-8. https://doi.org/10.3389/fpubh.2019.00277

Soltani, S. N., Kannaley, K., Tang, W., Gibson, A., Olscamp, K., Friedman, D. B., ... Hunter, R. H. (2017). Evaluating Community-Academic Partnerships of the South Carolina Healthy Brain Research Network. Health Promotion Practice, 18(4), 607-614. https://doi.org/10.1177/1524839917700086

Sorensen, G., Barbeau, E., Stoddard, A. M., Hunt, M. K., Kaphingst, K., \& Wallace, L. (2005). Promoting behavior change among working-class, multiethnic workers: Results of the healthy directions - Small business study. American Journal of Public Health, 95(8), 1389-1395. https://doi.org/10.2105/AJPH.2004.038745

Sorensen, G., Emmons, K., Hunt, M. K., \& Johnston, G. (1998). Implications of the results of community intervention trials. Public Health, 19, 379-416. 
Statistics coordinator of Grogol Petamburan sub-district. (2016). Statistics of Grogol Petamburan Sub-disctrict 2016. (Regional Neraca and Statistics Analysis Team, Ed.) (31740.1615). Jakarta: BPS - Statistics Jakarta Barat Municipality Office.

Statistics Indonesia. (2010). Population of Jakarta by Region and Citizenship Status. Retrieved January 7, 2016, from http://sp2010.bps.go.id/index.php/site/tabel?tid=323\&wid=3100000000

Statistics Indonesia. (2013). Health profile of Indonesia : number of general hospitals, special hospitals, and public health centers, 2012 and 2013. Retrieved January 7, 2016, from https://www.bps.go.id/linkTableDinamis/ view/id/933

Statistics Indonesia. (2015). Population of Indonesia: Result of the 2015 Intercensal Population Census. Jakarta. Statistics Indonesia. (2016). Ringkasan Eksekutif Ketenagakerjaan 2016 (Executive Summary) (Vol. 2016). Jakarta.

Steinberg, F. (2007). Jakarta: Environmental problems and sustainability. Habitat International, 31(3-4), 354-365. https://doi.org/10.1016/j.habitatint.2007.06.002

Stephens, C. (2008). Health Promotion: a psychosocial approach. (S. Payne \& S. Horn, Eds.). Berkshire: Open University Press. Retrieved from https://epdf.pub/health-promotion-a-psychosocial-approach-health-psychology. html

Stevenson, F. A., Britten, N., Barry, C. A., Bradley, C. P., \& Barber, N. (2003). Self-treatment and its discussion in medical consultations: How is medical pluralism managed in practice? Social Science and Medicine, 57(3), 513-527. https://doi.org/10.1016/S0277-9536(02)00377-5

Suarez-Balcazar, Y., Harper, G. W., \& Lewis, R. (2005). An interactive and contextual model of communityuniversity collaborations for research and action. Health Education and Behavior, 32(1), 84-101. https://doi. org/10.1177/1090198104269512

Suchert, V., Hanewinkel, R., \& Isensee, B. (2015). Sedentary behavior and indicators of mental health in schoolaged children and adolescents: A systematic review. Preventive Medicine, 76, 48-57. https://doi.org/10.1016/j. ypmed.2015.03.026

Suprianto, A., \& Mutiarin, D. (2017). Evaluasi Pelaksanaan Jaminan Kesehatan Nasional. Journal of Governance and Public Policy, 4(1), 11-19. https://doi.org/10.18196/jgpp.4172

Suryowati, E. (2016, August 16). Ini $15 \mathrm{~K} / \mathrm{L}$ dengan Anggaran Terbesar di RAPBN 2017. Kompas.Com. Retrieved from https://ekonomi.kompas.com/read/2016/08/16/204046326/ini.15.k.1.dengan.anggaran.terbesar. di.rapbn. 2017

Suswardany, D. L., Sibbritt, D. W., Supardi, S., Pardosi, J. F., Chang, S., \& Adams, J. (2017). A cross-sectional analysis of traditional medicine use for malaria alongside free antimalarial drugs treatment amongst adults in high-risk malaria endemic provinces of Indonesia. PLOS ONE, 12(3), 1-15. https://doi.org/10.1371/journal. pone. 0173522

Tamanam, J. V. (2016). Utilisation of Primary Health Care services: the perceptions and experiences of South Asian immigrants in Auckland, New Zealand. The University of Auckland. Retrieved from https://researchspace. auckland.ac.nz/bitstream/handle/2292/29143/whole.pdf?sequence=26

Tarigan, M. (2015, September 22). Anggaran Rp 4 Triliun, Puskesmas diminta fokus pencegahan. Tempo. Retrieved from https:/m.tempo.co/read/news/2015/09/22/173702948/anggaran-rp-4-triliun-puskesmas-diminta-fokuspencegahan\#UUIAh3deOOim3Upc.97

The President of the Republic of Indonesia. Act of the Republic of Indonesia number 36 of 2009, Pub. L. No. 36 of 2009 (2009). Republic of Indonesia: Health Ministry of the Republic of Indonesia.

The President of the Republic of Indonesia. The Law of the Republic of Indonesia number 24 of 2011, Pub. L. No. 24 of 2011, 24 (2011). Indonesia. https://doi.org/10.1017/CBO9781107415324.004

The President of the Republic of Indonesia. The Republic of Indonesia's Government Regulations number 47 of 2016, Pub. L. No. number 47 of 2016 (2016). The Republic of Indonesia: Health Ministry of the Republic of Indonesia.

Thom, D. H., \& Tirado, M. D. (2006). Development and validation of a patient-reported measure of physician cultural competency. Medical Care Research and Review, 63(5), 636-655. https://doi. org/10.1177/1077558706290946 


\section{References}

Thompson, S. J., \& Gifford, S. M. (2000). Trying to keep a balance: The meaning of health and diabetes in an urban Aboriginal community. Social Science and Medicine, 51(10), 1457-1472. https://doi.org/10.1016/ S0277-9536(00)00046-0

Tomkins, A., Duff, J., Fitzgibbon, A., Karam, A., Mills, E. J., Munnings, K., ... Yugi, P. (2015). Controversies in faith and health care. The Lancet, 386, 1776-1785. https://doi.org/10.1016/S0140-6736(15)60252-5

Treerutkuarkul, A., \& Gruber, K. (2015). Prevention is better than treatment. Bulletin of the World Health Organization, 9(93), 594-596. https://doi.org/http://dx.doi.org/10.2471/BLT.15.020915

Triratnawati, A. (2010). Pengobatan Tradisional, Upaya Meminimalkan Biaya Kesehatan Masyarakat Desa Di Jawa. Jurnal Manajemen Pelayanan Kesehatan, 13(2), 69-73. Retrieved from https://journal.ugm.ac.id/ jmpk/article/viewFile/2598/2329

Tristantia, I., \& Khoirunnisa, F. N. (2018). Kinerja kader kesehatan dalam pelaksanaan posyandu di Kabupaten Kudus. Jurnal Ilmu Keperawatan Dan Kebidanan Vol.9, 9(2), 192-199.

Tucker, C. M., Arthur, T. M., Roncoroni, J., Wall, W., \& Sanchez, J. (2015). Patient-Centered, Culturally Sensitive Health Care. American Journal of Lifestyle Medicine, 9(1), 63-77. https://doi.org/10.1177/1559827613498065

Tunas, D., \& Peresthu, A. (2010). The self-help housing in Indonesia: The only option for the poor? Habitat International, 34(3), 315-322. https://doi.org/10.1016/j.habitatint.2009.11.007

United Nations - Department of Economic and Social Affairs - Population Division. (2019). World Urbanization Prospects: The 2018 Revision (ST/ESA/SER.A/420). New York. https://doi.org/10.4054/demres.2005.12.9

US Department of Health and Human Services - Office of Minority Health. (2001). National Standards for Culturally and Linguistically Appropriate Services in Health Care: Executive Summary. Washington, DC. Retrieved from http://www.ingentaconnect.com/content/springer/cmanj/2002/00000003/00000002/art00005

van Empelen, P., Kok, G., Schaalma, H. P., \& Bartholomew, L. K. (2003). An AIDS Risk Reduction Program for Dutch Drug Users: An Intervention Mapping Approach to Planning. Health Promotion Practice, 4(4), 402-412. https://doi.org/10.1177/1524839903255421

van Oostrom, S. H., Anema, J. R., Terluin, B., Venema, A., de Vet, H. C. W., \& van Mechelen, W. (2007) Development of a workplace intervention for sick-listed employees with stress-related mental disorders: Intervention Mapping as a useful tool. BMC Health Services Research, 7, 127. https://doi.org/10.1186/14726963-7-127

Vaughn, L., Jacquez, F., \& Baker, R. (2009). Cultural health attributions, beliefs, and practices: Effects on healthcare and medical education. Open Medical Education Journal, 2, 64-74. Retrieved from http://www. benthamsciencepublisher.com/open/tomededuj/articles/V002/SI0016TOMEDEDUJ/64TOMEDEDUJ.pdf

Verbestel, V., De Henauw, S., Maes, L., Haerens, L., Mårild, S., Eiben, G., ... De Bourdeaudhuij, I. (2011). Using the intervention mapping protocol to develop a community-based intervention for the prevention of childhood obesity in a multi-centre European project: the IDEFICS intervention. Int J Behav Nutr Phys Act, 8(82), 1-15. https://doi.org/10.1186/1479-5868-8-82

Vermeulen, S. J., Anema, J. R., Schellart, A. J. M., van Mechelen, W., \& van der Beek, A. J. (2009). Intervention mapping for development of a participatory return-to-work intervention for temporary agency workers and unemployed workers sick-listed due to musculoskeletal disorders. BMC Public Health, 15, 1-15. https://doi. org/10.1186/1471-2458-9-216

Voestermans, P., \& Verheggen, T. (2013). Culture as embodiment: The social tuning of behavior. West Sussex: Wiley-Blackwell Publishing Ltd.

Watson, J., \& Platt, S. (Eds.). (2000). Researching health promotion. London: Routledge Taylor \& Francis Group. Retrieved from http://web.a.ebscohost.com/ehost/ebookviewer/ebook/ bmx1YmtfXzcyMzI1X19BTg2?sid=6dff6647-af64-4559-9997-862e6e095ef8@sdc-vsessmgr06\&vid $=0 \&$ format $=$ EB\&rid $=1$

Welcome to Country. (2018). Meet the Traditional Aboriginal Healers working inside Australia's hospitals Retrieved November 28, 2018, from https:/www.welcometocountry.org/traditional-aboriginal-healersaustralia-hospitals/?fbclid=IwAR30te4Zq-LarNqZB_whZGQJ5B0d5ZrOaIxFF74LDAtfWR-Riz7KuBr-Zdk 
Westmaas, A. H. (2013). Finding ways to promote STI testing among the Afro-Caribbean community in the Netherlands. Maastricht University, the Netherlands.

Whitehead, M., Pennington, A., Orton, L., Nayak, S., Petticrew, M., Sowden, A., \& White, M. (2016). How could differences in 'control over destiny' lead to socio-economic inequalities in health? A synthesis of theories and pathways in the living environment. Health \& Place, 39, 51-61. https://doi.org/10.1016/j. healthplace.2016.02.002

WHO. (1998). Health promotion glossary. World Health Organization (WHO) (WHO/HPR/HE). Geneva: World Health Organisation. https://doi.org/10.1093/heapro/1.1.113

WHO. (2013). WHO Traditional Medicine Strategy 2014-2023. World Health Organization (WHO). Hong Kong: World Health Organization (WHO). https://doi.org/2013

WHO. (2014). World health statistics 2014. World Health Organization. Geneva: World Health Organisation. https://doi.org/978 9241564588

WHO Country Office Indonesia. (2016). WHO country cooperation strategy 2014-2019: Indonesia. World Health Organization. India: The World Health Organization. https://doi.org/10.1007/s13398-014-0173-7.2

Widiyani, R. (2014). Dokter di Puskesmas Seharusnya Tak Sekadar Merujuk. Retrieved September 20, 2019 , from https://sains.kompas.com/read/2014/03/17/1600192/Dokter.di.Puskesmas.Seharusnya.Tak.Sekadar. Merujuk.

Willis, D. G., Sullivan-Bolyai, S., Knafl, K., \& Cohen, M. Z. (2016). Distinguishing Features and Similarities Between Descriptive Phenomenological and Qualitative Description Research. Western Journal of Nursing Research, 38(9), 1185-1204. https://doi.org/10.1177/0193945916645499

Winkleby, M. A., Howard-Pitney, B., Albright, C. A., Bruce, B., Kraemer, H. C., \& Fortmann, S. P. (1997) Predicting achievement of a low-fat diet: A nutrition intervention for adults with low literacy skills. Preventive Medicine, 26(6), 874-882. https://doi.org/10.1006/pmed.1997.0231

Witmer, L., Bocarro, J. N., \& Henderson, K. (2011). Adolescent Girls ' Perception of Health within a Leisure Context. Journal of Leisure Research, 43(3), 334-354. https://doi.org/10.1080/00222216.2011.11950240

Wong, A. (2015). Challenges for Asian health and Asian health promotion in New Zealand. Health Promotion Forum of New Zealand. Auckland. Retrieved from http://www.hauora.co.nz/ hpforum/assets/files/Occasional Papers/15128 FINAL Health promotion forum Asian promotion article.pdf

Woodyatt, C. R., Finneran, C. A., \& Stephenson, R. (2016). In-Person Versus Online Focus Group Discussions: A Comparative Analysis of Data Quality. Qualitative Health Research, 26(6), 741-749. https://doi. org/10.1177/1049732316631510

Xu, J., \& Yang, Y. (2009). Traditional Chinese medicine in the Chinese health care system. Health Policy, $90(2-$ 3), 133-139. https://doi.org/10.1016/j.healthpol.2008.09.003

Yanek, L. R., Becker, D. M., Moy, T. F., Gittelsohn, J., \& Koffman, D. M. (2001). Project joy: Faith based cardiovascular health promotion for African American women. Public Health Reports, 116(SUPPL. 1), 6881. https://doi.org/10.1093/phr/116.S1.68

Yang, E., Foster-Fishman, P., Collins, C., \& Ahn, S. (2012). Testing a comprehensive community problemsolving framework for community coalitions. Journal of Community Psychology, 40(6), 681-698. https:// doi.org/DOI: $10.1002 /$ jcop.20526

Young, S., \& Guo, K. L. (2016). Cultural diversity training: The necessity of cultural competence for health care providers and in nursing practice. Health Care Manager, 35(2), 94-102. https://doi.org/10.1097/ HCM.0000000000000100

Zakocs, R. C., \& Edwards, E. M. (2006). What explains community coalition effectiveness? A review of the literature. American Journal of Preventive Medicine, 30(4), 351-361. https://doi.org/10.1016/j. amepre.2005.12.004 


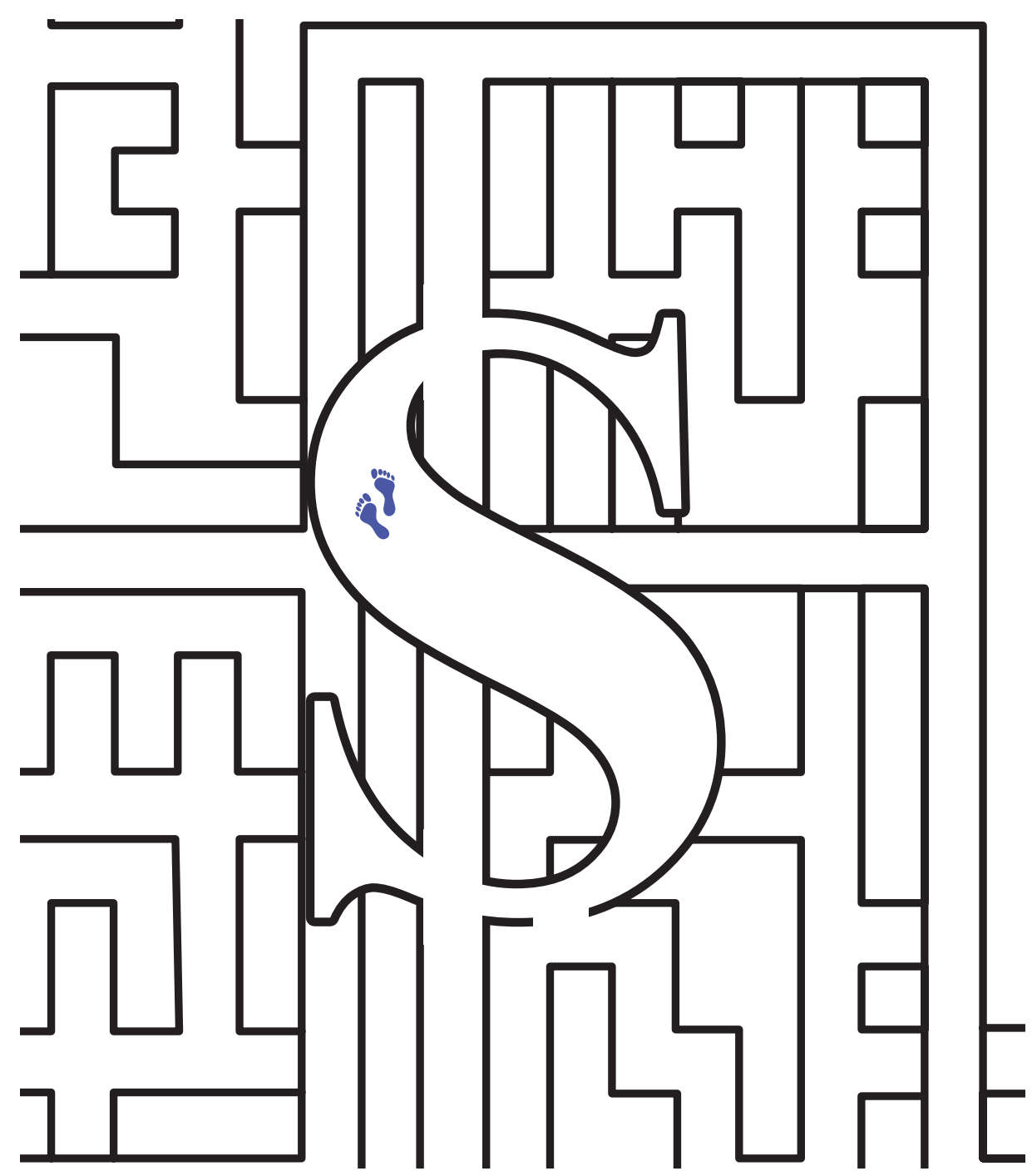




\section{Summary/Samenvatting/ Intisari}

Note:

The Dutch translation was provided by Dr. A. Westmaas. The Indonesian translation was provided by Dr. Wisnu Wiradhany. I thank them. 


\section{A starting mark of health promotion in Jakarta: voicing the community's perspective}

\section{Summary}

In spite of the ongoing health promotion efforts in in Jakarta as the capital city of Indonesia, the health status of Jakartans (i.e., the citizens of Jakarta city) is not better than that in the other regions of Indonesia. This suggests that the existing health promotion programs have not been effective. One of the main reasons behind this ineffectiveness is believed to be that the primary health service providers in Jakarta, namely the Community Health Centers (CHCs; Indonesian: Pusat Kesehatan Masyarakat, Puskesmas), give too much attention to the biomedical perspective of health (i.e., health as the consequence of physical changes in the body), and give low priority to the links between health and social-cultural aspects of community. At the same time, it has been shown that understanding and appreciating the socio-cultural aspects of health issues is crucial for the development of health promotion plans with sound, effective, and sustainable interventions and programs.

Considering the possible gap above between health care users and health care providers in Jakarta, the aim of this dissertation is to unravel the socio-cultural values regarding health and health promotions of Jakartans, which include the health care users and health care providers. This goal was broken down into these research questions: 1) What do health and sickness actually mean to Jakartans? 2) How do health care users in Jakarta perceive the quality of health services provided? 3) What are the challenges and obstacles for conducting health promotion programs in Jakarta from the perspective of the health workers? 4) What are the aspects considered important in applying the Community Coalition approach (CC; a group of individuals from diverse organizations, or constituencies within the community who agree to work as a team to achieve a common goal) for health promotion intervention in urban areas?

With regard to the meaning of health and sickness according to Jakartans, the results indicate that Jakartans had a multidimensional view on health and sickness. These views include the spiritual aspects, the (in)ability to do daily work aspects, and the combination of several aspects, such as a combination of physical, psychological and the (in)ability to do daily activities aspects. For instance, a worker who emphasizes health as the ability to do daily activities would continue to work in spite of having a cold, in spite of the risk of spreading the disease to their colleagues. We discussed how this multidimensional view may affect how Jakartans maintain their health and treat their sicknesses. In addition, we also discussed how the biomedical perspective adopted by health providers is unable to accommodate these multidimensional health-related needs of Jakartans. 
The third chapter investigates the perspectives of Jakartans on the quality of health care services. Jakartans had a negative view on CHCs, the primary health care providers in Indonesia. This negative view originated from their experience comparing CHCs, private clinics, and hospitals. Among the three, CHCs provide the most economical treatments, but at the same time Jakartans did not feel comfortable with the service due to the unfriendly staff, long queues, short service hours, and the perceived low quality of medicines and facilities. On the other hand, Jakartans were hesitant to visit private clinics or hospitals because of their higher treatment cost. As a way out, Jakartans choose to do self-treatment or consume traditional medicines before they visit CHCs. These findings indicate that the primary health care services are currently unable to accommodate the health needs of Jakartans.

To complement the views of the community regarding health care services, in the fourth chapter, we investigated health care workers' point of view on health care services. Specifically, we explored the challenges and obstacles encountered by health workers in conducting health promotion programs in Jakarta. Similar to health care users, we found that health care workers were also not satisfied with the programs they have. Health care workers were understaffed due to responsibility to provide services both inside and outside of $\mathrm{CHCs}$, but at the same time, the number of patients at $\mathrm{CHCs}$ remained high. Due to the amount of time they had to spend for treating patients, $\mathrm{CHC}$ workers had limited time to conduct health promotion or education program in the community. In line with the findings from our previous study, another challenge was the tendency of Jakartans to administer self- and traditional treatments, and only visit CHCs or a general practitioner when their health condition is worsening. Health care workers were aware of these challenges and obstacles, and they proposed a collaboration with community members, such as health volunteers, and religious leaders to develop a better health promotion program. Furthermore, health care workers also suggested the Indonesian government to establish a special team with the task of providing health care services and promotions outside the CHCs.

Following the suggestions above, we found the $\mathrm{CC}$ approach as the most appropriate approach to respond to the need for collaborations between health care users and workers. Therefore, in the fifth chapter we conducted a literature review to highlight important aspects to consider when implementing the $\mathrm{CC}$ approach in urban areas. The review highlights seven important aspects, namely: (1) the diverse expertise of the members, (2) the clarity of the coalition's goal, (3) the member's interest and motivation, (4) the leadership, (5) the trust and cohesion of the coalition member, (6) the clarity of the member's roles, and (7) understanding the norm and the culture of the target community.

In summary, Jakartans have a multidimensional view of health yet, they cannot find health care services that are suitable for fulfilling their health-related needs when they use health care services in Jakarta, especially in CHCs. Consequently, Jakartans took matters in their own hands, for example by using self-and traditional treatments to treat themselves. At the same time, health workers at CHCs were too understaffed and overworked to conduct proper health promotion and education (e.g., about the risks 
and benefits of traditional treatments) programs in Jakarta. To promote a more holistic approach in health promotion programs in Jakarta, we proposed the implementation of a CC approach using the Intervention Mapping process in a series of recommendations to stakeholders in Jakarta, including health researchers, the Indonesian government, health care service providers, and policy makers. In implementing our suggestions, we advise Jakarta's stakeholders to adopt evidence-based approaches, while at the same time prioritizing and voicing the cultural values of the community. 


\section{Een startpunt voor gezondheidsbevordering in Jakarta: het perspectief van de gemeenschap uitdrukken}

\section{Samenvatting}

Ondanks de voortdurende inspanningen voor gezondheidsbevordering in Jakarta, als de hoofdstad van Indonesië, is de gezondheidstoestand van Jakartanen (d.w.z. de inwoners van de stad Jakarta) niet beter dan die in de andere regio's van Indonesië. Dit suggereert dat de bestaande gezondheidspromotieprogramma's niet effectief zijn geweest. Het wordt verondersteld dat een van de belangrijkste redenen voor deze ineffectiviteit voort komt uit de grote aandacht die geschonken wordt aan het biomedische perspectief van gezondheid (dat wil zeggen: gezondheid als gevolg van fysieke veranderingen in het lichaam), en het geven van een lage prioriteit aan de verbanden tussen gezondheid en sociaal-culturele aspecten van de gemeenschap. Tegelijkertijd is aangetoond dat het begrijpen en waarderen van de sociaalculturele aspecten van gezondheidsproblemen cruciaal is voor de ontwikkeling van gezondheidsbevorderingsplannen met degelijke, effectieve en duurzame interventies en programma's.

Gezien de mogelijke kloof tussen zorgvragers en zorgverleners in Jakarta, is het doel van dit proefschrift om de sociaal-culturele waarden met betrekking tot gezondheid en gezondheidspromoties van Jakartanen te doorgronden, met in achtneming van zorgvragers en zorgverleners. Dit doel werd onderverdeeld in de volgende onderzoeksvragen: 1) 'Wat betekenen gezondheid en ziekte eigenlijk voor Jakartanen?', 2) 'Hoe zien zorgvragers de kwaliteit van de geboden gezondheidsdiensten?', 3) 'Wat zijn de uitdagingen en obstakels voor het uitvoeren van gezondheidspromotieprogramma's in Jakarta vanuit het perspectief van de zorgverlener?', en 4) 'Wat zijn de aspecten die belangrijk worden geacht bij de toepassing van de gemeenschapscoalitiebenadering (Engels: Community Coalition; een groep personen uit verschillende organisaties, of kiesdistricten binnen de gemeenschap die overeenkomen om als een team te werken om een gemeenschappelijk doel te bereiken) voor gezondheidsbevordering in stedelijke gebieden?'

Wat betreft de betekenis van gezondheid en ziekte (hoofdstuk 1), geven de resultaten aan dat Jakartans een multidimensionale kijk op gezondheid en ziekte hadden. Deze opvattingen omvatten de spirituele aspecten, het (on) vermogen om dagelijkse werkaspecten te doen, en de combinatie van verschillende aspecten, zoals een combinatie van fysieke, psychologische en het (on) vermogen om dagelijkse activiteitenaspecten te doen. Een werknemer die bijvoorbeeld de nadruk legt op gezondheid als het vermogen om dagelijkse activiteiten te doen, zou blijven werken ondanks een verkoudheid, ondanks het risico van verspreiding van de ziekte naar 
zijn collega's. We hebben besproken hoe deze multidimensionale visie van invloed kan zijn op hoe Jakartanen hun gezondheid behouden en hun ziekten behandelen. Tevens hebben we ook besproken hoe het biomedische perspectief van zorgaanbieders niet in staat is om tegemoet te komen aan deze multidimensionale gezondheidsgerelateerde behoeften van Jakartanen (hoofdstuk 2).

Het derde hoofdstuk beschrijft de exploratie van de perspectieven van Jakartanen op de kwaliteit van de gezondheidszorg. Jakartanen hadden een negatieve kijk op het gemeenschapsgezondheidscentrum, de primaire zorgverleners in Indonesië. Deze negatieve kijk is ontstaan uit hun ervaringen bij het vergelijken van gezondheidscentra, privéklinieken en ziekenhuizen. Van de drie biedt het gemeenschapsgezondheidscentrum de meest economische behandelingen, maar tegelijkertijd voelden Jakartanen zich niet op hun gemak bij de service vanwege het onvriendelijke personeel, lange wachtrijen, korte service-uren en de waargenomen lage kwaliteit van medicijnen en voorzieningen. Aan de andere kant aarzelden Jakartanen om privéklinieken of ziekenhuizen te bezoeken vanwege hun hogere behandelingskosten. Als uitweg kozen Jakartanen ervoor om aan zelfbehandeling te doen of traditionele medicijnen te consumeren, voordat ze het gezondheidscentrum bezochten. Deze bevindingen geven aan dat de eerstelijnsgezondheidszorg momenteel niet kan voorzien in de gezondheidsbehoeften van Jakartanen.

Als aanvulling op de opvattingen van de gemeenschap met betrekking tot gezondheidszorg, hebben we in het vierde hoofdstuk het standpunt van zorgverleners over gezondheidszorg onderzocht. Specifiek hebben we de uitdagingen en obstakels onderzocht die zorgverleners tegenkomen bij het uitvoeren van gezondheidsbevorderende programma's in Jakarta. Net als de zorgvragers hebben we vastgesteld dat zorgverleners ook niet tevreden waren met de programma's die ze hebben. Medewerkers in de gezondheidszorg waren onderbezet vanwege de verantwoordelijkheid om zowel binnen als buiten het gezondheidscentrum diensten te verlenen, maar tegelijkertijd bleef het aantal patiënten in het gezondheidscentrum hoog. Vanwege de hoeveelheid tijd die ze moesten besteden aan de behandeling van patiënten, hadden zorgverleners in de gemeenschap beperkte tijd om gezondheidsbevordering of een educatief programma in de gemeenschap uit te voeren. In overeenstemming met de bevindingen van onze vorige studie, was een andere uitdaging de neiging van Jakartanen om zelf- en traditionele behandelingen toe te passen en alleen een gemeenschapscentrum of een huisarts te bezoeken wanneer hun gezondheidstoestand verslechterde. Zorgverleners waren zich bewust van deze uitdagingen en obstakels en ze stelden een samenwerking voor met leden van de gemeenschap, zoals gezondheidsvrijwilligers, en religieuze leiders om een beter gezondheidsbevorderingsprogramma te ontwikkelen. Bovendien stelden gezondheidswerkers de Indonesische regering voor om een speciaal team op te richten met als taak zorgdiensten en promoties buiten het gezondheidscentrum van de gemeenschap aan te bieden.

Naar aanleiding van de bovenstaande suggesties, vonden we de benadering van de gemeenschapscoalitie de meest geschikte aanpak om te reageren op de behoefte aan 
samenwerking tussen zorgvragers en zorgverleners. Daarom hebben we in het vijfde hoofdstuk een literatuuronderzoek uitgevoerd om belangrijke aspecten te benadrukken waarmee rekening moet worden gehouden bij de implementatie van de aanpak van gemeenschapscoalities in stedelijke gebieden. De literatuurstudie benadrukt zeven belangrijke aspecten, namelijk: (1) de uiteenlopende expertise van de leden, (2) de duidelijkheid van het doel van de coalitie, (3) de interesse en motivatie van het lid, (4) het leiderschap, (5) het vertrouwen en samenhang van het coalitielid, (6) de duidelijkheid van de rollen van het lid, en (7) inzicht in de norm en de cultuur van de doelgemeenschap.

Samenvattend hadden Jakartanen een multidimensionale kijk op gezondheid, maar ze konden geen gezondheidszorg vinden die geschikt was om aan hun gezondheidsgerelateerde behoeften te voldoen wanneer ze gezondheidszorg in Jakarta gebruikten, vooral in het gemeenschapsgezondheidscentrum. Als gevolg nemen Jakartanen het heft in eigen handen, bijvoorbeeld door zelf- en traditionele behandelingen te gebruiken om zichzelf te behandelen. Tegelijkertijd waren gezondheidswerkers in het gemeenschapsgezondheidscentrum onderbezet en te overwerkt om goede gezondheidsbevordering en voorlichting te geven (bijvoorbeeld over de risico's en voordelen van traditionele behandelingen) in Jakarta. Om een meer holistische benadering in gezondheidspromotieprogramma's in Jakarta te bevorderen, stelden we de implementatie voor van een gemeenschapscoalitiebenadering met behulp van het Intervention Mapping-proces in een reeks aanbevelingen aan belanghebbenden in Jakarta, waaronder gezondheidsonderzoekers, de Indonesische overheid, zorgverleners, en beleidsmakers. Bij de uitvoering van onze suggesties adviseren we de stakeholders van Jakarta om op feiten gebaseerde benaderingen te hanteren, terwijl we tegelijkertijd de culturele waarden van de gemeenschap prioriteren en uiten. 


\section{Langkah Awal Promosi Kesehatan di Jakarta: Menyuarakan Perspektif Masyarakat}

\section{Intisari}

Terlepas dari upaya promosi kesehatan yang dilakukan di Jakarta sebagai ibu kota negara, status kesehatan warga Jakarta tidak lebih baik dari wilayah lain di Indonesia. Ini menunjukkan bahwa program promosi kesehatan yang selama ini dilakukan belumlah efektif. Diyakini, salah satu alasan utama di balik ketidakefektifan ini adalah penyedia layanan kesehatan di Jakarta, terutama Pusat Kesehatan Masyarakat (Puskesmas), terlalu berfokus pada perspektif biomedis (yaitu, kesehatan sebagai konsekuensi dari perubahan fisik dalam tubuh), dan mereka kurang memprioritaskan kaitan kesehatan dengan aspek sosial-budaya masyarakat. Pada saat yang sama, berbagai penelitian menunjukkan bahwa memahami dan menghargai aspek sosial-budaya dari isu kesehatan sangatlah penting untuk mengembangkan rencana promosi kesehatan dengan intervensi dan program yang tepat, efektif, dan berkelanjutan.

Mempertimbangkan adanya kesenjangan antara pengguna dan penyedia layanan kesehatan di Jakarta, tujuan disertasi ini adalah mengungkap nilai-nilai sosial-budaya yang berkaitan dengan kesehatan dan promosi kesehatan warga Jakarta, termasuk pengguna dan penyedia layanan kesehatan. Tujuan ini terbagi menjadi pertanyaan penelitian: 1) Apa makna sehat dan sakit bagi warga Jakarta? 2) Bagaimana para pengguna layanan kesehatan memahami kualitas layanan kesehatan yang diberikan di Jakarta? 3) Apa saja tantangan dan hambatan dalam melakukan program promosi kesehatan di Jakarta dari perspektif petugas kesehatan? 4) Apa saja aspek yang dianggap penting dalam menerapkan pendekatan Koalisi Komunitas (Bahasa Inggris: Community Coalition; yaitu, sekelompok individu dari organisasi atau kelompok yang berbeda-beda dalam komunitas yang setuju untuk bekerja sama sebagai tim untuk mencapai sebuah tujuan yang sama) untuk intervensi promosi kesehatan di daerah perkotaan?

Berkenaan dengan makna sehat dan sakit menurut warga Jakarta, hasil penelitian menunjukkan bahwa warga Jakarta memiliki pandangan multidimensional mengenai sehat dan sakit. Pandangan multidimensional ini mencakup aspek spiritual, aspek ke(tidak) mampuan melakukan pekerjaan sehari-hari, dan kombinasi dari beberapa aspek, seperti kombinasi aspek fisik, psikologis dan ke(tidak)mampuan melakukan pekerjaan seharihari. Sebagai contoh, seorang karyawan yang memaknai sehat sebagai kemampuan untuk melakukan kegiatan sehari-hari akan terus masuk kerja meskipun terkena flu, terlepas dari adanya risiko dia akan menyebarkan penyakit ke rekan kerjanya. Dalam disertasi ini, kami membahas bagaimana pandangan multidimensional ini dapat memengaruhi cara warga Jakarta menjaga kesehatan dan mengobati penyakit mereka. Selain itu, kami juga membahas bagaimana perspektif biomedis yang diadopsi oleh penyedia layanan kesehatan tidak mampu mengakomodasi kebutuhan multidimensi kesehatan warga Jakarta tersebut di atas. 
Bab ketiga meneliti perspektif warga Jakarta terhadap kualitas layanan kesehatan yang ada di Jakarta. Warga Jakarta memiliki pandangan negatif terhadap Puskesmas sebagai penyedia layanan kesehatan utama di Indonesia. Pandangan negatif ini berasal dari pengalaman mereka membandingkan Puskesmas, klinik swasta, dan rumah sakit. Di antara ketiganya, Puskesmas memberikan jasa perawatan yang paling murah, tetapi pada saat yang sama warga Jakarta merasa tidak nyaman karena staf yang tidak ramah, antrian yang panjang, jam pelayanan yang singkat, dan mereka menganggap rendah kualitas obat-obatan dan fasilitas di Puskesmas. Di sisi lain, warga Jakarta enggan mendatangi klinik swasta atau rumah sakit karena biaya perawatan yang lebih mahal. Sebagai jalan keluar, warga Jakarta memilih untuk melakukan pengobatan sendiri atau mengonsumsi obat-obatan tradisional sebelum mereka pergi ke Puskesmas. Temuan ini menunjukkan bahwa layanan perawatan kesehatan utama yang ada saat ini tidak dapat mengakomodasi kebutuhan kesehatan warga Jakarta.

Untuk melengkapi pandangan masyarakat tentang layanan perawatan kesehatan, pada bab keempat, kami meneliti sudut pandang petugas kesehatan terhadap layanan perawatan kesehatan. Secara khusus, kami mengeksplorasi tantangan dan hambatan yang dihadapi oleh petugas kesehatan dalam melakukan program promosi kesehatan di Jakarta. Mirip dengan pengguna layanan kesehatan, kami menemukan bahwa petugas kesehatan juga tidak puas dengan program yang mereka miliki. Petugas kesehatan mengalami kekurangan staf karena mereka bertanggung jawab untuk menyediakan layanan baik di dalam maupun di luar Puskesmas, padahal jumlah pasien di Puskesmas tinggi. Karena sebagian besar waktu petugas kesehatan digunakan untuk merawat pasien, mereka kekurangan waktu untuk melakukan promosi kesehatan atau program edukasi di masyarakat. Sejalan dengan temuan dari penelitian kami sebelumnya, menurut petugas kesehatan, warga Jakarta cenderung melakukan pengobatan sendiri atau menggunakan obat tradisional, dan hanya datang ke Puskesmas atau dokter ketika kondisi kesehatannya sudah memburuk. Petugas kesehatan menyadari tantangan dan hambatan ini, dan mereka mengusulkan kolaborasi dengan anggota masyarakat, seperti kader kesehatan dan pemimpin agama untuk mengembangkan program promosi kesehatan yang lebih baik. Selain itu, petugas kesehatan juga menyarankan kepada pemerintah Indonesia untuk membentuk tim khusus dengan tugas menyediakan layanan perawatan kesehatan dan promosi di luar Puskesmas.

Mengikuti saran di atas, kami menyarankan pendekatan Koalisi Komunitas sebagai pendekatan yang paling ideal untuk menanggapi kebutuhan kolaborasi antara pengguna dan pekerja layanan kesehatan. Dalam bab kelima kami melakukan tinjauan literatur untuk menyoroti aspek-aspek penting yang perlu dipertimbangkan dalam penerapan pendekatan Koalisi Komunitas di daerah perkotaan. Tinjauan ini menekankan tujuh aspek penting, yaitu: (1) keahlian beragam para anggota, (2) kejelasan tujuan koalisi, (3) minat dan motivasi anggota, (4) kepemimpinan, (5) kepercayaan dan kohesi anggota koalisi, (6) kejelasan peran anggota, dan (7) memahami norma dan budaya masyarakat yang menjadi komunitas sasaran. 
Secara singkat dapat dikatakan bahwa warga Jakarta memiliki pandangan multidimensional mengenai kesehatan, mereka tidak dapat menemukan layanan perawatan kesehatan yang cocok untuk memenuhi kebutuhan tersebut ketika menggunakan layanan perawatan kesehatan di Jakarta, terutama di Puskesmas. Akibatnya, warga Jakarta mengambil tindakan sendiri, sebagai contoh dengan melakukan pengobatan sendiri atau mengonsumsi obat-obatan tradisional untuk mengobati sakitnya. Pada saat yang sama, petugas kesehatan di Puskesmas sangat kekurangan tenaga dan memiliki terlalu banyak pekerjaan untuk dapat melakukan program promosi dan edukasi kesehatan secara tepat (misalnya, mengenai risiko dan manfaat perawatan tradisional) di Jakarta. Sebagai pendekatan yang lebih menyeluruh dalam program promosi kesehatan di Jakarta, kami mengusulkan penerapan pendekatan Koalisi Komunitas menggunakan proses Pemetaan Intervensi (Bahasa Inggris: Intervention Mapping) melalui serangkaian rekomendasi kepada pemangku kepentingan di Jakarta, termasuk peneliti kesehatan, pemerintah Indonesia, penyedia layanan kesehatan, dan pembuat kebijakan. Dalam mengimplementasikan usul ini, kami memberi saran kepada para pemangku kepentingan di Jakarta untuk melakukan pendekatan berbasis bukti-bukti ilmiah, sambil memprioritaskan dan menyuarakan nilai-nilai budaya yang ada di masyarakat. 


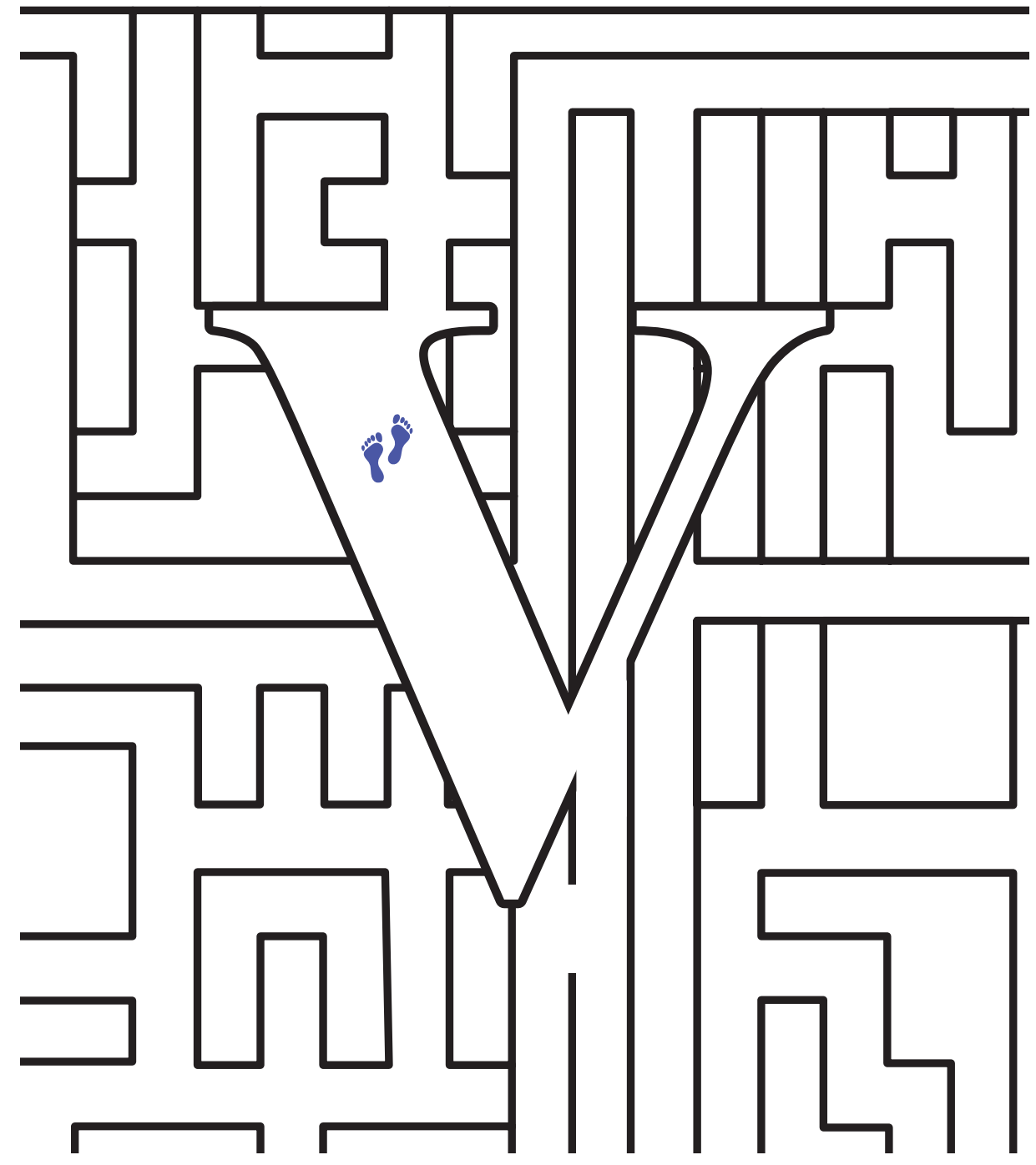


Valorization 
The focus of this dissertation is on understanding the important values regarding health and health promotion by community members from Jakarta, including health-care users and workers, while paying attention to the cultural context of these issues. Here, we provide a brief description of the practical implications and recommendations for future studies. We will explain the target group and a step-by-step implementation program that could potentially be developed from this dissertation.

The studies featured in this dissertation were conducted at Grogol Petamburan sub-district, West Jakarta Municipality, Indonesia. Thus, the members of the Grogol Petamburan community are the main target of the health promotion intervention using the Community Coalition approach, which includes religious leaders and health cadres/ volunteers. Further, the intervention program would also provide benefit for the Jakarta government and policy-makers. Additionally, the intervention targets health providers, health practitioners, health researchers, and health promoters who are interested in applying a culturally-sensitive approach in health promotion programs.

This dissertation provides a number of future practical recommendations aimed at different stages of implementation of the intervention. The first step is to disseminate the information regarding the results of this dissertation to Jakarta stakeholders, such as the government of Jakarta, especially the Grogol Petamburan sub-district officials, Community Health Centers (CHCs; Indonesian: Pusat Kesehatan Masyarakat, Puskesmas), health practitioners, health researchers, health promoters, and the member of Jakartan communities, including, but not limited to, religious leaders and health cadres/volunteers (i.e., community members who are chosen by community members and work voluntarily to maintain the health level of a their community).

The next step is to establish and work with a planning group, including subdistrict (Indonesian: kecamatan) and village (Indonesian: kelurahan) officials as the representatives of Jakarta government and policy makers, and CHC staff e.g. general practitioners, nurses, and midwives, clinical psychologists, health researchers from universities, head of community associations (Indonesian: rukun warga $R W$ ), head of neighborhood associations (Indonesian: rukun tetangga, $R T$ ), health cadres/volunteers, religious leaders, and community members. These stakeholders will act as members of the community coalition.

After this coalition is established, the coalition members would discuss the goals of the coalition. At the same time, the members need to clarify the interest and motivation of each member, leadership and staffing, as well as the structure of the coalition to clarify the member's roles. The coalition goals can be further specified into sections, such as short-term, medium-term and long-term goals.

The short-term goals extend the first step of Intervention Mapping framework to reach a wider range of participants by using questionnaires or surveys that are designed based on our initial studies. The next step is to frame a logic model of change by incorporating evidence-based insights to develop appropriate health promotion interventions for Jakartans. The aim is to develop a blueprint of interventions in accordance with the shared decisions among coalition members. The intervention blueprint can be used as 
a proposal to obtain funds, for example, from the Ministry of Health of the Republic of Indonesia, the Health Office of Jakarta Municipality, the Ministry of Research and Technology/National Research and Innovation Agency of the Republic of Indonesia, and other resources, such as from corporate social responsibility funding, non-governmental organizations, or crowd funding.

After the coalition acquires funding, the focus can be turned to medium-term goals, which is creating a program design based on the above-mentioned blueprint. The coalition can focus on several subjects, such as:

1) Building a special 'field' team to focus on health promotion in the targeted community, as mentioned in the Chapter 4 and has been studied on Chapter 5 of this dissertation. The special team members include e.g. a clinical psychologist, health cadres/volunteers, religious leader, and a medical staff such as a general practitioner, nurse, or a midwife. The coalition provides training for the special team members so that they would fulfill their specific roles that are in line with their own interest and motivation.

2) Reinforcing the implementation of the Indonesian Health Minister' regulation number 44 of 2018 regarding the involvement of hospitals in health promotion efforts (Ministry of Health Republic of Indonesia, 2018) to support CHCs roles in giving health promotion and education programs in community.

3) Disseminating the regulation regarding Indonesia's traditional medicines in the Indonesian Health Minister's regulation number HK.01.07/MENKES/187/2017 (Ministry of Health Republic of Indonesia, 2017) in order to change health care workers negative perception toward traditional medicine. The coalition will support the Indonesian government in disseminating health regulations and policies to Jakarta communities through its partnerships. Thus, the spread of information will be faster and the Jakarta government will overcome the lack of human resources at CHCs.

The long-term goals of the coalition would focus on step 4, 5, and 6 of the Intervention Mapping framework, which aims to develop program materials production, program implementation plan, and program evaluation plan. The progress and achievement of short- and medium- term goals will determine the implementation of steps 4, 5, and 6. However, despite having six steps, the Intervention Mapping frameworks is an iterative process. In the years ahead, we expect to develop health policies and regulations based on the implementation of health promotion interventions mentioned to bridge the gap between the challenges faced by health care providers and the needs of the Jakarta communities. 


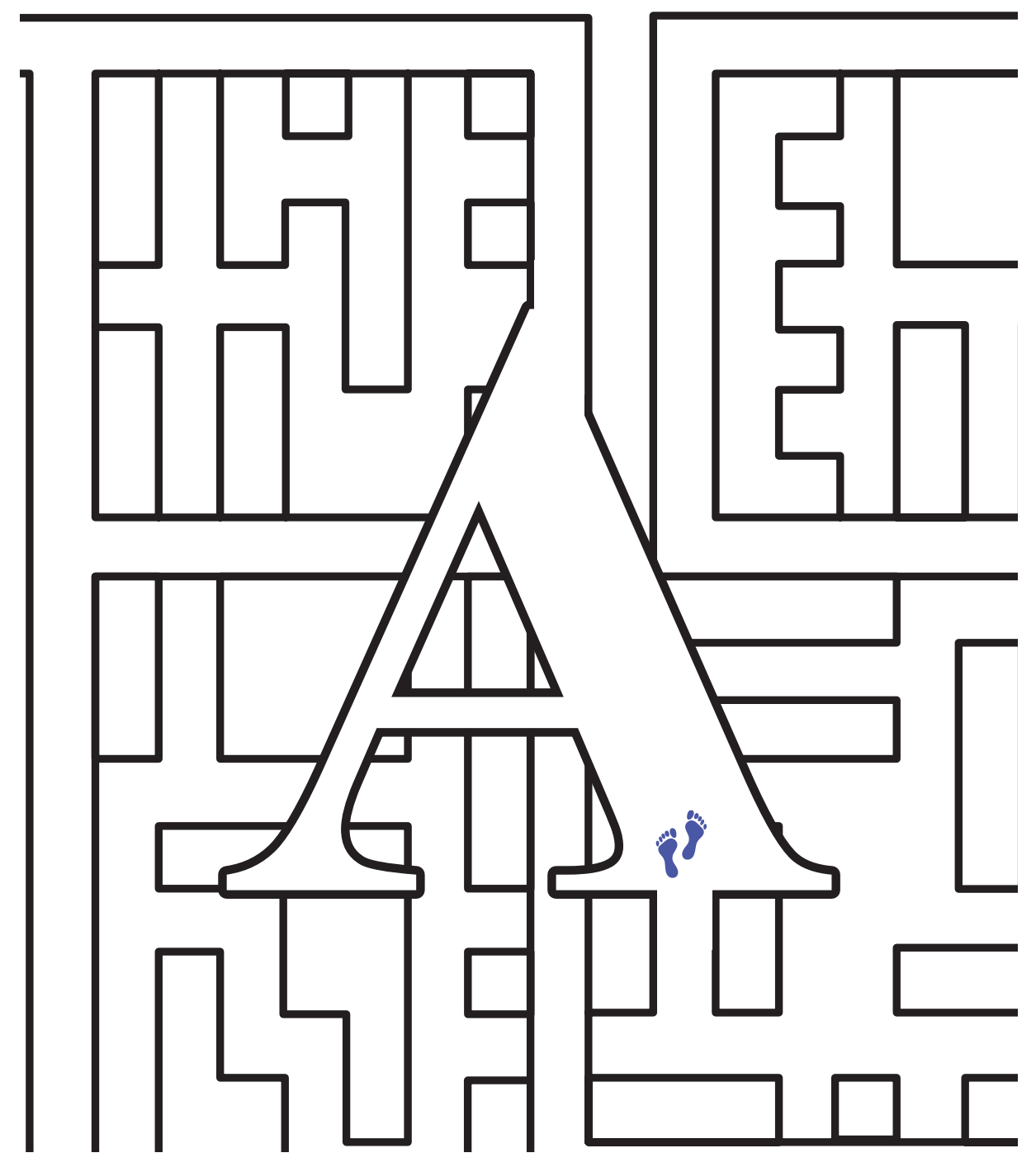


Acknowledgements 
I would like to present this section to all the wonderful people who have supported me, directly and indirectly, in any forms and ways. This book and this project would not have been possible without your support. I feel blessed to have met and known each of you.

I wish to express my sincere gratitude to my supervisors, Gerjo and Alvin, who trusted me from the beginning. Thank you very much for giving me the opportunity to pursue my PhD. Thank you for always encouraging me with the brainstorming and discussion sessions. Both of you kept challenging me to think outside of the box, while providing me a safe environment in the process. It gave me confidence to believe in myself. I would also like to acknowledge LPDP (Indonesia Endowment Fund for Education) for funding this $\mathrm{PhD}$ project. Without the scholarship, I would not be at this point today. The project also would not have been possible without the great help from my research assistants: Valen, Ferdi, Josephine Ondja, Sugeng, Maisie, Iatri, Afi, and Ika.

I would like to give my special regards to Rob Ruiter, the first person I met when I came to Maastricht. Thank you for agreeing to be my Chair for the defense, all the 'Hi, Astin' and for the praise whenever I bring Indonesian food to the croissant time on Friday. My living-abroad comrade, Merve. Thank you for all of our lunches, shared moments, and talks at the neighbor's canteen. Marie, Bram, and Abbas for the talks and fun discussions, especially in the evening, when our concentration is getting low. Alicia Sui, thank you for our unforgettable journey in Croatia, all the discussions and our heart-to-heart sharing. Gerda and Annet, thank you for encouraging me to do tutorials. It gave me insights on being a lecturer abroad, and I will definitely bring back those insights back to Indonesia. Herco, thank you for inviting me for discussion about Indonesia in your class. It was such a good experience for me to see how your students worked with Indonesian students. Thank you, all the members of Work and Social Department: Mart, Alicia Walkowiak, Rosine, Fleurie, Sjir, Philippe, Karlijn, Gemma, Sarah, Karen, Phil Brüll, Nathalie, Ute, Kai, Irma, Fraukje, Annika, Margje, Fred, Robert, Trudy, Joedith, Darta, Nino, and Khalid.

I would also like to give my special gratitude to Mariella. Thank you for helping me with so many things. Thank you for your sincerity, long before we even met, moreover in person. Sylvia Roozen, thank you for the friendship since the first day I came to the university. For our memorable walk to the nature side of Maastricht and Valkenburg. And thank you for being our wedding witness. Henna Toppenberg, you are one of my first friends in Maastricht. You helped me a lot during my first months when most of the faculty members were on summer holidays.

Many thanks to all my Indonesian friends, Nael, Alia, Jozua, Bobby, Untung, Bona, Tari for every discussion and senasib sepenanggungan feeling. To all Indonesian students at PPIM, thank you for your friendship. To all my BC Rally friends, especially Oom Jacky, Martijn, Jos, Francis, Esther, Marisca for your friendliness, all the fun badminton games, and your understanding on my (still) not improving Dutch. Gill, thank you for being part of my review committee. Thank you also for all fun badminton game we sometimes played at BC Rally. Thank you, Leon, and the badminton friends at UM Sport for the fun, stress releasing moment after working hours. 
I would like to give my special gratitude to the whole Psychology Faculty of Krida Wacana Christian University team. To JEP, thank you for your support and encouragement ever since the first time we met. Thank you also for being one of my review committee members. The other members of the faculty, Sandjaja, Olivia, Anita, Zadok, Sukma, Pinkan, Denny, Jovita, Evans, Sherly, Petra, William, and Ngadiman, thank you for your support regardless of the difference in continent and time zone. Also, to give me the chance to pursuit my $\mathrm{PhD}$ regardless 'the waiting line'.

I wish to acknowledge the support and great love from my family, my Mom, Katarina Limba, thank you for always trusting my decisions. My brothers (in-law) and sisters (inlaw), my beautiful nieces and nephews. I love you all. Last but definitely not the least, my dearest Wisnu, for all your patience and care for me. You always encourage and support me in every possible way. Thank you for being with me in my best and worst time. It made me feel safe and keep me moving forward.

I would also like to share my life principle: Masakatsu Agatsu. Masakatsu and Agatsu are two of principles in Aikido training. Aikido is one of Japanese martial arts developed by Morihei Ueshiba with goal to defend oneself while protecting the attacker(s) from injury.

Masakatsu means victory that comes from the correct method. One is not training to become stronger or to hurt their opponent. Its purpose is to unify oneself with the universe to bring peace in the world. Agatsu means to have victory over one's self. An understanding of Masakatsu is important to practice Agatsu. The Agatsu principle shows that for those who look at their true self, the most fearful opponent is no one other than themselves. It says, for those who are unable to help themselves, there is no way they can help others.

I shared these two words as it gave a deep meaning to me. The principles are relevant, not only for martial arts, but also -at least, from my perspective- for life in general. I like Aikido martial arts and its principles; and I had a chance to learn it years ago. Therefore, I wish to express my sincere deepest gratitude to myself for my effort to always learning - fight, lose, and win - over my own self. I hope to practice the Masakatsu Agatsu in everyday life and in every aspects of my life. 


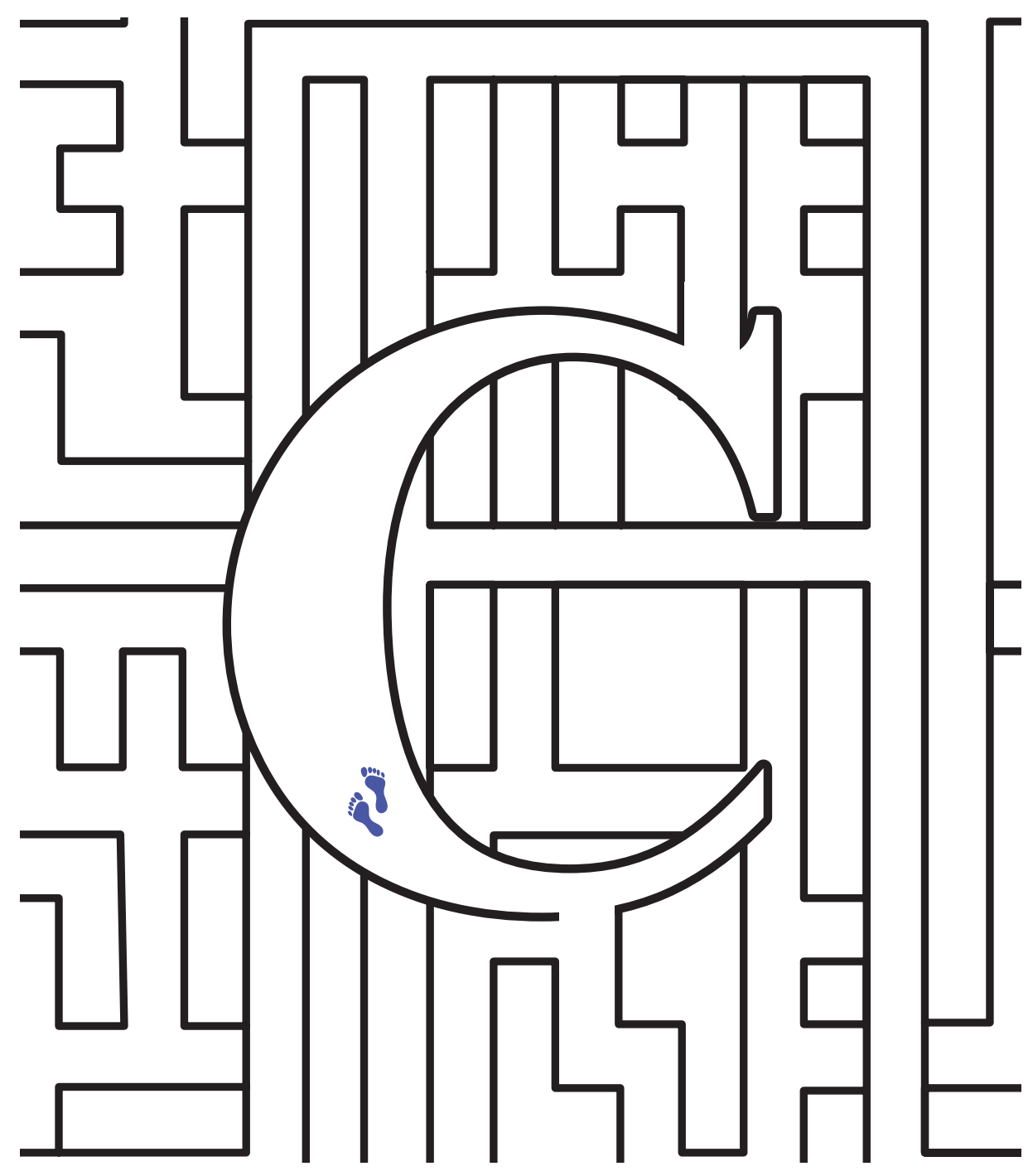




\section{Curriculum Vitae}


Yasinta Astin Sokang was born in Ruteng, Flores Island, Indonesia on September 19, 1985. She finished her BSc in Psychology at Sanata Dharma University, Jogjakarta in 2008. Right after graduation, she worked for two years as an assistant psychologist and addiction counselor at Kedhaton Parahita Center for Drug Addiction Recovery, Sentul City, Indonesia. In 2010, she enrolled in the Master of Clinical Psychology and Clinical Psychologist program of Universitas Gadjah Mada, Yogyakarta, Indonesia, which she finished in 2012. She moved to Jakarta at the same year to work at the Krida Wacana Christian University as a lecturer and as a secretary and program manager of the Center for Research and Community. She moved to the Netherlands in 2016 to work on her PhD project at the Department of Work and Social Psychology, Faculty of Psychology and Neuroscience, Maastricht University. This project is fully funded by the Indonesia Endowment Fund for Education, Ministry of Finance of the Republic of Indonesia. This project examines the socio-cultural values regarding health and health promotions in Jakarta, Indonesia. During the $\mathrm{PhD}$, she also worked as a tutor at the Faculty of Psychology and Neuroscience, Maastricht University. 


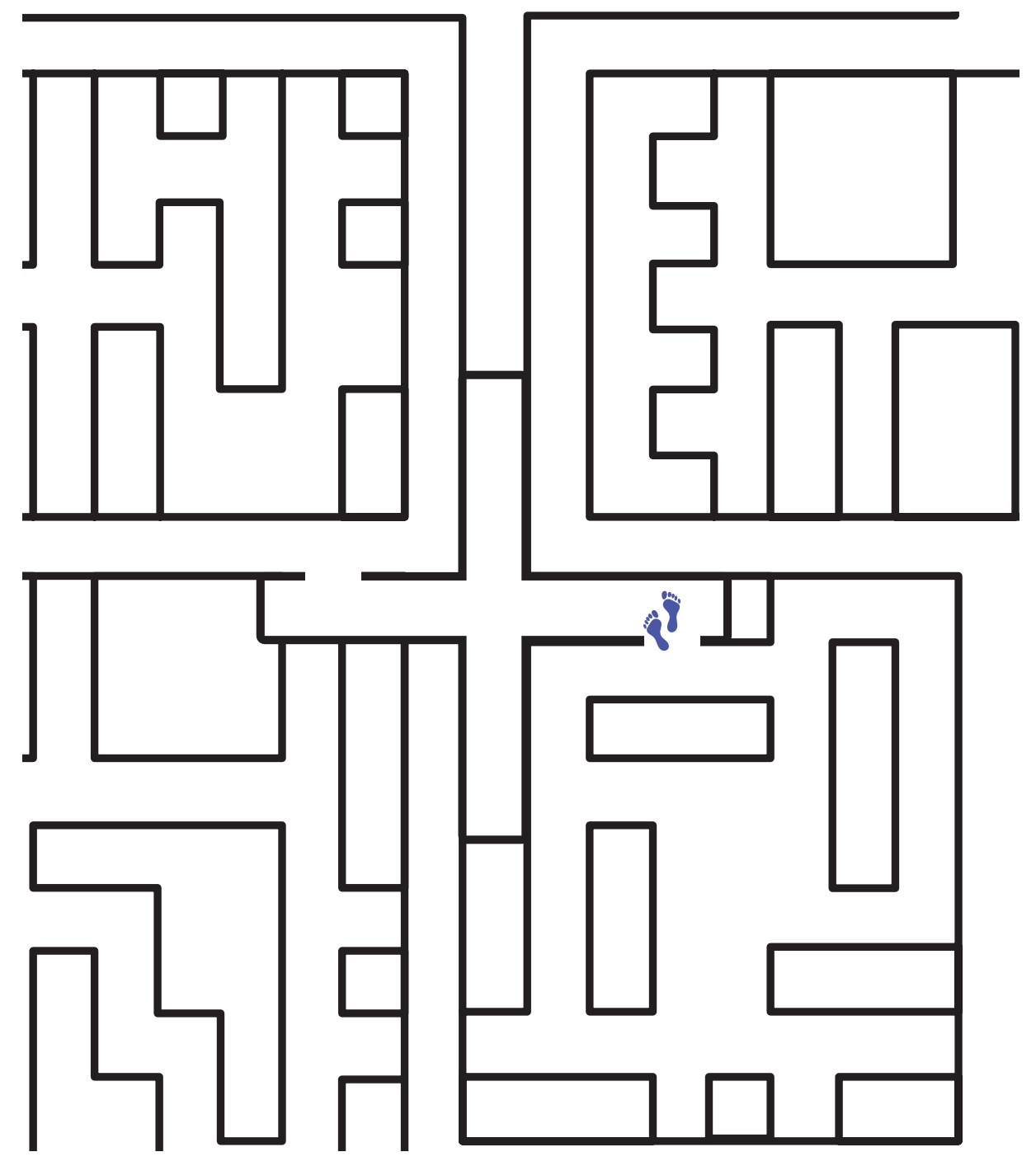


Appendix 


\section{Appendix A: Coding Rules for 'Single-Term' and 'In Combination' Categories}

\section{A.1. Single-Term Category}

Single-Term category means that respondents perceived their health and/or sickness based on one single condition of health/sickness, so that the statement could be put under one category. If respondents mentioned more than two conditions that could be included in one category, the statement was coded as one answer in one category.

\section{A.2. In Combination Category}

In combination category means that the respondents' statements were based on many conditions of health/sickness that could be allocated to two or more categories. If respondents mentioned more than one theme in their answer, the response was recorded as In Combination categories. As a consequence, the number of responses exceeds the number of respondents.

\section{Appendix B: Coding Rules to Make Categories in Relation to the Meaning of Health}

\section{B.1. Physical Condition}

This category includes responses that mentioned body, body-parts, physical condition, organs, immune system (including hormones and antibodies), having good stamina, being pain-free, being free from bacteria, viruses, and disease.

\section{B.2. Psychological Condition}

This category includes answers that described psychological condition, including feelings (for example, being comfortable, happy, jubilant, fresh, calm, happy, peaceful, joyful), being sober [sane], free of stress, safe, free of problems, without any burden, in a good situation, and free of suffering.

\section{B.3. Spiritual Condition}

This category includes statements that mentioned spirit, spiritual, soul, God, faith, and blessing.

\section{B.4. Capability to carry out Daily Activities}

This category includes sentences that referred to an activity (or activities)/ a daily activity (or activities), capability/ ability to carry out a daily activity (or activities), ability to do something such as work, have a job, and to be productive. 


\section{B.5. Healthy Lifestyles}

This category includes comments that referred to a healthy lifestyle, healthy diet, dietary habits (including eating and drinking habits), exercise, sleep, and a clean environment.

\section{Appendix C: Coding Rules to Make Categories in Relation to the Meaning of Sickness}

\section{C.1. Physical Condition}

This category includes responses that referred to body, body-parts, organs, physical condition, immune system, bacteria, viruses, physical disease, pain, stamina, hormones, and antibodies.

\section{C.2. Psychological Condition}

This category includes answers that referred to psychological condition, mental disorders, feelings (including sadness, suffering, distress), thoughts and problems (including perception, having a lot of things one one's mind, not being able to find a way out), feeling unsafe, and life burden.

\section{C.3. Abnormal Circumstances and Bad Situations}

This category includes statements that signified bad situations or abnormal circumstances; something that did not work properly, did not occur as usual, was not normal, or was out of the ordinary.

\section{C.4. Spiritual Condition}

This category includes comments that mentioned spirit, spiritual, soul, God, temptation, curse, and sickness occurring as the result of sin.

\section{C.5. Inability to Carry out Daily Activities}

This category includes sentences that indicated activity (or activities), daily activity (or activities) inability to carry out daily activities, or being distracted when performing daily activities inability to work, or being distracted when doing something such as work, not being productive. 


\title{
Appendix D: Sentence examples for Economic Single Term of Health
}

\author{
'Invaluable treasure' (male, 20 years old) \\ 'Most valuable treasure' (male, 55 years old) \\ 'A lot of money' (male, 19 years old) \\ 'The most beautiful treasure'(female 19 years old) \\ 'An expensive thing and must be maintained' (female 19 years old)
}

\section{Appendix E: Sentence examples for Unhealthy Lifestyle single term of Sickness}

'When we consume foods that cannot gives [contribute to] health to our body, like fried foods, etc.' (female, 22 years old)

'Dirty environment, lack of cleanliness' (female, 45 years old)

'A situation or event that suffered or experienced when we did not live healthy (careless and arbitrary)' (female, 19 years old)

'Situation where self cannot live healthy and do not take care of own health' (female, 20 years old)

'Lack of exercise, less clean or dirty environment and less regular sleep, and can cause illness or lack of drinking water' (male, 26 years old)

\section{'Bad lifestyle' (male, 18 years old)}

'As a result of unable [to] keep the diet, irregular sleep, etc.' (male, 21 years old) 


\section{Appendix F: Sentence examples for Economic single term of Sickness}

'Adverse conditions so we have to pay something'(male, 27 years old)

'Abnormal circumstances [that happened] to someone which incur losses'(male, 19 years old)

'No money' (male, 19 years old)

'Expensive' (female, 28 years old)

'Non-productive condition' (female, 28 years old)

'Biggest losses'(female, 18 years old) 